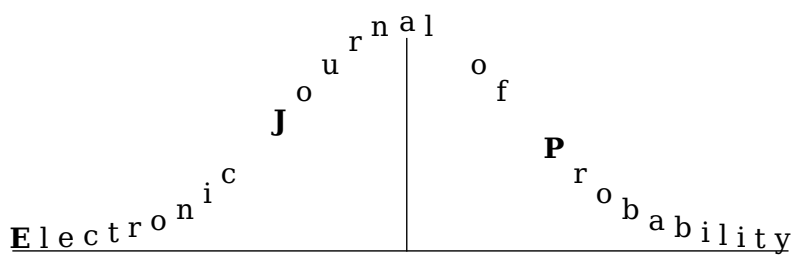

Electron. J. Probab. 27 (2022), article no. 29, 1-40.

ISSN: 1083-6489 https://doi.org/10.1214/22-EJP758

\title{
On the non-asymptotic concentration of heteroskedastic Wishart-type matrix ${ }^{*}$
}

\author{
T. Tony $\mathrm{Cai}^{\dagger} \quad$ Rungang $\mathrm{Han}^{\ddagger} \quad$ Anru R. Zhang ${ }^{\S}$
}

\begin{abstract}
This paper focuses on the non-asymptotic concentration of the heteroskedastic Wisharttype matrices. Suppose $Z$ is a $p_{1}$-by- $p_{2}$ random matrix and $Z_{i j} \sim N\left(0, \sigma_{i j}^{2}\right)$ independently, we prove the expected spectral norm of Wishart matrix deviations (i.e., $\left.\mathbb{E}\left\|Z Z^{\top}-\mathbb{E} Z Z^{\top}\right\|\right)$ is upper bounded by

$$
(1+\epsilon)\left\{2 \sigma_{C} \sigma_{R}+\sigma_{C}^{2}+C \sigma_{R} \sigma_{*} \sqrt{\log \left(p_{1} \wedge p_{2}\right)}+C \sigma_{*}^{2} \log \left(p_{1} \wedge p_{2}\right)\right\}
$$

where $\sigma_{C}^{2}:=\max _{j} \sum_{i=1}^{p_{1}} \sigma_{i j}^{2}, \sigma_{R}^{2}:=\max _{i} \sum_{j=1}^{p_{2}} \sigma_{i j}^{2}$ and $\sigma_{*}^{2}:=\max _{i, j} \sigma_{i j}^{2}$. A minimax lower bound is developed that matches this upper bound. Then, we derive the concentration inequalities, moments, and tail bounds for the heteroskedastic Wisharttype matrix under more general distributions, such as sub-Gaussian and heavy-tailed distributions. Next, we consider the cases where $Z$ has homoskedastic columns or rows (i.e., $\sigma_{i j} \approx \sigma_{i}$ or $\sigma_{i j} \approx \sigma_{j}$ ) and derive the rate-optimal Wishart-type concentration bounds. Finally, we apply the developed tools to identify the sharp signal-to-noise ratio threshold for consistent clustering in the heteroskedastic clustering problem.
\end{abstract}

Keywords: concentration inequality; nonasymptotic bound; random matrix; Wishart matrix. MSC2020 subject classifications: Primary 60B20, Secondary 46B09.

Submitted to EJP on January 4, 2021, final version accepted on February 16, 2022.

\section{Introduction}

Random matrix theory is an important topic in its own right and has been proven to be a powerful tool in a wide range of applications in statistics, high-energy physics, and number theory. Wigner matrices, symmetric matrices with mean-zero independent and

\footnotetext{
${ }^{*}$ The research of Tony Cai was supported in part by NSF grants DMS-1712735 and DMS-2015259 and NIH grants R01-GM129781 and R01-GM123056. The research of Rungang Han and Anru R. Zhang was supported in part by NSF CAREER-1944904, NSF DMS-1811868, and NIH R01-GM131399.

${ }^{\dagger}$ University of Pennsylvania, United States of America. E-mail: tcai@wharton . upenn.edu

${ }^{\ddagger}$ Duke University, United States of America. E-mail: rungang. han@duke. edu

${ }^{\S}$ University of Wisconsin-Madison and Duke University, United States of America. E-mail: anru. zhang@duke. edu
} 
identically distributed (i.i.d.) entries (subject to the symmetry constraint), have been a particular focus. Asymptotic and non-asymptotic properties of the spectrum of Wigner matrices have been widely studied in the literature. See, for example, [2, 28, 31] and the references therein.

Motivated by a range of applications, heteroskedastic Wigner-type matrices, random matrices with independent heteroskedastic entries, have attracted much recent attention. A central problem of interest is the characterization of the dependence of the spectral norm $\|\cdot\|$ (i.e., the largest singular value of the matrix) of a heteroskedastic Wignertype matrix on the variances of its entries. To answer this question, Ajanki, Erdős, Krüger [1] established the asymptotic behavior of the resolvent, a local law down to the smallest spectral resolution scale, and bulk universality for the heteroskedastic Wigner-type matrix. Bandeira and van Handel [4] proved an non-asymptotic upper bound for the spectral norm. More specifically, let $Z=\left(Z_{i j}\right)$ be a $p \times p$ heteroskedastic Wigner-type matrix with $\operatorname{Var}\left(Z_{i j}\right):=\sigma_{i j}^{2}$. Bandeira and van Handel [4] showed that $\mathbb{E}\|Z\| \lesssim \sigma+\sigma_{*} \sqrt{\log p}$, where $\sigma^{2}=\max _{i} \sum_{j} \sigma_{i j}^{2}$ and $\sigma_{*}^{2}=\max _{i j} \sigma_{i j}^{2}$ are the column-sumwise and entry-wise maximum variances, respectively. This bound was improved by van Handel [30] to $\mathbb{E}\|Z\| \lesssim \sigma+\max _{i, j \in[p]} \sigma_{i j}^{*} \log i$. Here, the matrix $\left\{\sigma_{i j}^{*}\right\}$ is obtained by permuting the rows and columns of the variance matrix $\left\{\sigma_{i j}\right\}$ such that $\max _{j} \sigma_{1 j}^{*} \geq$ $\max _{j} \sigma_{2 j}^{*} \geq \cdots \geq \max _{j} \sigma_{p j}^{*}$. Later, Latała and van Handel [20] further improved it to a tight bound:

$$
\mathbb{E}\|Z\| \asymp \sigma+\max _{i, j \in[p]} \sigma_{i j}^{*} \sqrt{\log i} .
$$

In addition to the Wigner-type matrix, the Wishart-type matrix, $Z Z^{\top}-\mathbb{E} Z Z^{\top}$, also plays a crucial role in many high-dimensional statistical problems, including the principal component analysis (PCA) and factor analysis [36], matrix denoising [25], and bipartite community detection [15]. Though there have been many results on the asymptotic and non-asymptotic properties of the homoskedastic Wishart-type matrix, where $Z$ has i.i.d entries (see [8] for an introduction and the references therein), the properties of the heteroskedastic Wishart-type matrices are much less understood.

Specifically, suppose $Z$ is a $p_{1} \times p_{2}$ random matrix with independent and zeromean entries. In this paper, we are interested in the Wishart-type concentration: $\mathbb{E}\left\|Z Z^{\top}-\mathbb{E} Z Z^{\top}\right\|$. Define $\sigma_{C}^{2}, \sigma_{R}^{2}, \sigma_{*}^{2}$ as the column-sum-wise, row-sum-wise, and entrywise maximum variances:

$$
\sigma_{C}^{2}=\max _{j} \sum_{i=1}^{p_{1}} \sigma_{i j}^{2}, \quad \sigma_{R}^{2}=\max _{i} \sum_{j=1}^{p_{2}} \sigma_{i j}^{2}, \quad \sigma_{*}^{2}=\max _{i j} \sigma_{i j}^{2} .
$$

By the symmetrization scheme and the asymmetric Wigner-type concentration inequality in [4], it is not difficult to show that

$$
\begin{aligned}
& \mathbb{E}\left\|Z Z^{\top}-\mathbb{E} Z Z^{\top}\right\| \leq \mathbb{E}\left\|Z Z^{\top}-Z^{\prime}\left(Z^{\prime}\right)^{\top}\right\| \leq 2 \mathbb{E}\left\|Z Z^{\top}\right\|=2 \mathbb{E}\|Z\|^{2} \\
& \lesssim\left(\sigma_{C}+\sigma_{R}+\sigma_{*} \sqrt{\log \left(p_{1} \wedge p_{2}\right)}\right)^{2} .
\end{aligned}
$$

Since $Z Z^{\top}-\mathbb{E} Z Z^{\top}$ can be decomposed into a sum of independent random matrices,

$$
Z Z^{\top}-\mathbb{E} Z Z^{\top}=\sum_{j=1}^{p_{2}}\left(Z_{\cdot j} Z_{\cdot j}^{\top}-\mathbb{E} Z_{\cdot j} Z_{\cdot j}^{\top}\right)
$$

one can apply the concentration inequality for the sum of independent random matrices [29, Theorem 1] to show that

$$
\mathbb{E}\left\|Z Z^{\top}-\mathbb{E} Z Z^{\top}\right\| \lesssim \sigma_{C} \sigma_{R} \sqrt{\log p_{2}}+\sigma_{C}^{2}\left(\log p_{2}\right)^{2} .
$$


However, as we will show later, these bounds are not tight.

In this paper, we establish non-asymptotic bounds for the Wishart-type concentration $\mathbb{E}\left\|Z Z^{\top}-\mathbb{E} Z Z^{\top}\right\|$. The main results include the following. We begin by focusing on the Gaussian case in Section 2.1 and prove that if all entries of $Z$ are independently Gaussian,

$$
\mathbb{E}\left\|Z Z^{\top}-\mathbb{E} Z Z^{\top}\right\| \leq 2.01 \sigma_{C} \sigma_{R}+1.01 \sigma_{C}^{2}+C_{1} \sigma_{R} \sigma_{*} \sqrt{\log \left(p_{1} \wedge p_{2}\right)}+C_{2} \sigma_{*}^{2} \log \left(p_{1} \wedge p_{2}\right),
$$

where $C_{1}, C_{2}$ are some universal constants that does not depend on the variance components $\sigma_{C}, \sigma_{R}, \sigma_{*}$ or matrix dimensions $p_{1}, p_{2}$. Moreover, we can set the coefficients in front of $\sigma_{C} \sigma_{R}$ and $\sigma_{C}^{2}$ arbitrarily close to 2 and 1, respectively, at the sacrifice of larger constants $C_{1}, C_{2}$ in (1.5) (see Theorem 2.1 for details).

We further justify that the constants in $2 \sigma_{C} \sigma_{R}+\sigma_{C}^{2}$ are essential under the homoskedastic setting. The proof of (1.5) is based on a Wishart-type moment method provided in Section 2.2. In Section 2.3, we provide a lower bound to show that the upper bound (1.5) is minimax rate-optimal in a general class of heteroskedastic random matrices.

We then consider the more general non-Gaussian setting including sub-Gaussian, sub-exponential, heavy tailed, and bounded distributions in Section 3.1. In particular, we establish the following concentration bound when the entries have independent sub-Gaussian distributions:

$$
\mathbb{E}\left\|Z Z^{\top}-\mathbb{E} Z Z^{\top}\right\| \lesssim\left(\sigma_{C}+\sigma_{R}+\sigma_{*} \sqrt{\log \left(p_{1} \wedge p_{2}\right)}\right)^{2}-\sigma_{R}^{2} .
$$

Upper bounds for the moments and probability tails of $\left\|Z Z^{\top}-\mathbb{E} Z Z^{\top}\right\|$ are developed in Section 3.2.

In Sections 3.3 and 3.4, we consider two variance structures arising in statistical applications and develop tight Wishart-type concentration bounds. If the random matrix $Z$ has independent sub-Gaussian entries and homoskedastic rows, i.e., $\sigma_{i j}=\sigma_{i}$, we prove that

$$
\mathbb{E}\left\|Z Z^{\top}-\mathbb{E} Z Z^{\top}\right\| \asymp \sum_{i=1}^{p_{1}} \sigma_{i}^{2}+\sqrt{p_{2} \sum_{i=1}^{p_{1}} \sigma_{i}^{2}} \cdot \max _{i \in\left[p_{1}\right]} \sigma_{i} .
$$

If $Z$ has independent sub-Gaussian entries and homoskedastic column variances, i.e., $\sigma_{i j} \asymp \sigma_{j}$, we prove that

$$
\mathbb{E}\left\|Z Z^{\top}-\mathbb{E} Z Z^{\top}\right\| \asymp \sqrt{p_{1} \sum_{j=1}^{p_{2}} \sigma_{j}^{4}}+p_{1} \max _{j \in\left[p_{2}\right]} \sigma_{j}^{2} .
$$

To illustrate the usefulness of the newly established tools, we apply these tools in Section 4 to solve a statistical problem in heteroskedastic clustering. Specifically, we obtain a sharp signal-to-noise ratio threshold to guarantee consistent clustering.

\section{Main results}

We first introduce the notation to be used in the rest of the paper. Let $a \wedge b$ and $a \vee b$ be the minimum and maximum of real numbers $a$ and $b$, respectively. We use $[d]$ to denote the set $\{1, \ldots, d\}$ for any positive integer $d$. For any vector $v$, let $\|v\|_{q}=\left(\sum_{i}\left|v_{i}\right|^{q}\right)^{1 / q}$ be the vector $\ell_{q}$ norm; specifically, $\|v\|_{\infty}=\sup _{i}\left|v_{i}\right|$. For any sequences $\left\{a_{n}\right\},\left\{b_{n}\right\}$, denote $a \lesssim b$ (or $b_{n} \gtrsim a_{n}$ ) if there exists a uniform constant $C>0$ such that $a \leq C b$. If $a \lesssim b$ and 
$a \gtrsim b$ both hold, we say $a \asymp b$. For any $\alpha \geq 1$, the Orlicz $\psi_{\alpha}$ norm of any random variable $X$ is defined as

$$
\|X\|_{\psi_{\alpha}}=\inf \left\{x \geq 0: \mathbb{E} \exp \left((|X| / x)^{\alpha}\right) \leq 2\right\} .
$$

In the literature [31, 33], a random variable is often called sub-Gaussian, sub-exponential, or sub-Weibull with tail parameter $(1 / \alpha)$, if $\|X\|_{\psi_{2}} \leq C,\|X\|_{\psi_{1}} \leq C$, and $\|X\|_{\psi_{\alpha}} \leq C$, respectively. The matrix spectral norm is defined as $\|X\|=\sup _{u, v} \frac{u^{\top} X v}{\|u\|_{2}\|v\|_{2}}$. The capital letters $C, C_{1}, \tilde{C}$ and lowercase letters $c, c_{1}, c_{0}$ represent the generic large and small constants, respectively, whose exact values may vary from place to place.

\subsection{Concentration of heteroskedastic Wishart matrix}

We begin by considering the Gaussian case where the entries $Z_{i j} \sim N\left(0, \sigma_{i j}^{2}\right)$ independently. The following theorem provides an upper bound for the concentration and is one of the main results of the paper.

Theorem 2.1 (Wishart-type Concentration for Gaussian random matrix). Suppose $Z$ is a $p_{1}$-by- $p_{2}$ random matrix and $Z_{i j} \sim N\left(0, \sigma_{i j}^{2}\right)$ independently. Then for any $\epsilon_{1}, \epsilon_{2}>0$,

$$
\begin{aligned}
& \mathbb{E}\left\|Z Z^{\top}-\mathbb{E} Z Z^{\top}\right\| \\
\leq & \left(1+\epsilon_{1}\right)\left\{2 \sigma_{C} \sigma_{R}+\left(1+\epsilon_{2}\right) \sigma_{C}^{2}+C_{1}\left(\epsilon_{1}\right) \sigma_{R} \sigma_{*} \sqrt{\log \left(p_{1} \wedge p_{2}\right)}+C_{2}\left(\epsilon_{1}, \epsilon_{2}\right) \sigma_{*}^{2} \log \left(p_{1} \wedge p_{2}\right)\right\},
\end{aligned}
$$

where $C_{1}\left(\epsilon_{1}\right)=10\left(1+\epsilon_{1}\right) \sqrt{\left\lceil 1 / \log \left(1+\epsilon_{1}\right)\right\rceil}$ and $C_{2}\left(\epsilon_{1}, \epsilon_{2}\right)=\left(1+\epsilon_{1}\right)\left\lceil 1 / \log \left(1+\epsilon_{1}\right)\right\rceil$ $\left(\frac{25}{\epsilon_{2}}+24\right)$.

Remark 2.2 (Lower bound for the homoskedastic case). If $Z$ has independent and homoskedastic Gaussian entries, i.e., $Z_{i j} \stackrel{i i d}{\sim} N(0,1)$, then $\sigma_{C}=\sqrt{p_{1}}, \sigma_{R}=\sqrt{p_{2}}$, and Theorem 2.1 implies

$$
\mathbb{E}\left\|Z Z^{\top}-\mathbb{E} Z Z^{\top}\right\| \leq(1+\epsilon)\left(\sigma_{C}^{2}+2 \sigma_{C} \sigma_{R}\right)+C_{\epsilon} \sigma_{R} \sqrt{\log \left(p_{1} \wedge p_{2}\right)}
$$

for any $\epsilon>0$ and constant $C_{\epsilon}$ only depending on $\epsilon$. On the other hand, we have

Proposition 2.3. If $Z$ is a $p_{1}-b y-p_{2}$ matrix with i.i.d. homoskedastic Gaussian entries, then

$$
\liminf _{p_{1}, p_{2} \rightarrow \infty} \frac{\mathbb{E}\left\|Z Z^{\top}-\mathbb{E} Z Z^{\top}\right\|}{2 \sigma_{C} \sigma_{R}+\sigma_{C}^{2}} \geq 1 .
$$

Proposition (2.3) and (2.2) together indicate that $\left(\sigma_{C}^{2}+2 \sigma_{C} \sigma_{R}\right)$ in the upper bound of Theorem 2.1 are sharp in the homoskedastic case. In Section 2.3, we establish a minimax lower bound to show that all four terms in the upper bound (1.6) are essential when $Z$ is a general heteroskedastic random matrix.

\subsection{Proof of Theorem 2.1}

The proof of Theorem 2.1 relies on a moment method and the following fact: for a $p$-by- $p$ symmetric matrix $A$ (in the context of Theorem 2.1, $A=Z Z^{\top}-\mathbb{E} Z Z^{\top}$ ) and a even number $q \asymp \log (p)$, we have

$$
\|A\| \approx\left(\operatorname{tr}\left(A^{q}\right)\right)^{1 / q} .
$$

We introduce two lemmas for the proof of Theorem 2.1. First, Lemma 2.4 builds a comparison between the $q$-th moment of the heteroskedastic Wishart-type matrix $Z Z^{\top}-\mathbb{E} Z Z^{\top}$ with a homoskedastic analogue $H H^{\top}-\mathbb{E} H H^{\top}$. The complete proof of Lemma 2.4 is postponed to Section 5.1. 
Lemma 2.4 (Gaussian Comparison). Suppose $Z \in \mathbb{R}^{p_{1} \times p_{2}}$ has independent Gaussian entries: $Z_{i j} \sim N\left(0, \sigma_{i j}^{2}\right)$. Let $m_{1}=\left\lceil\sigma_{C}^{2}\right\rceil+q-1$ and $m_{2}=\left\lceil\sigma_{R}^{2}\right\rceil+q-1$. Suppose $H \in \mathbb{R}^{m_{1} \times m_{2}}$ has i.i.d. $N(0,1)$ entries. Then for any $q \geq 2$,

$$
\mathbb{E} \operatorname{tr}\left\{\left(Z Z^{\top}-\mathbb{E} Z Z^{\top}\right)^{q}\right\} \leq\left(\frac{p_{1}}{m_{1}} \wedge \frac{p_{2}}{m_{2}}\right) \mathbb{E} \operatorname{tr}\left\{\left(H H^{\top}-\mathbb{E} H H^{\top}\right)^{q}\right\} .
$$

Remark 2.5 (Proof sketch of Lemma 2.4). Previously, [4, Proposition 2.1] compared the moments of the Wigner-type matrices (i.e., $Z$ is symmetric and thus $p_{1}=p_{2}=p, \sigma_{C}=$ $\left.\sigma_{R}=\sigma\right)$ by the expansion $\mathbb{E} \operatorname{tr}\left(Z^{2 p}\right)=\sum_{u_{1}, \ldots, u_{2 q}} \mathbb{E}\left(Z_{u_{1} u_{2}} Z_{u_{2} u_{3}} \cdots Z_{u_{2 p} u_{1}}\right)$ and counting the cycles in a reduced unipartite graph:

$$
\mathbb{E} \operatorname{tr}\left(Z^{2 q}\right) \leq \frac{p}{\left\lceil\sigma^{2}\right\rceil+q} \mathbb{E} \operatorname{tr}\left(H^{2 q}\right) .
$$

Compared to the expansion of Wigner-type random matrix $\operatorname{Etr}\left(Z^{2 q}\right)$, the expansion of Wishart-type random matrix $\mathbb{E} \operatorname{tr}\left\{\left(Z Z^{\top}-\mathbb{E} Z Z^{\top}\right)^{q}\right\}$ is much more complicated:

$$
\begin{aligned}
& \operatorname{Etr}\left\{\left(Z Z^{\top}-\mathbb{E} Z Z^{\top}\right)^{q}\right\}=\sum_{\substack{u_{q+1}=u_{1}, \ldots, u_{q} \in\left[p_{1}\right] \\
v_{1}, \ldots, v_{q} \in\left[p_{2}\right]}} \mathbb{E} \prod_{k=1}^{q}\left(Z_{u_{k}, v_{k}} Z_{u_{k+1}, v_{k}}-\sigma_{u_{k}, v_{k}}^{2} \cdot 1_{\left\{u_{k}=u_{k+1}\right\}}\right) \\
& =\cdots=\sum_{\mathbf{c} \in\left(\left[p_{1}\right] \times\left[p_{2}\right]\right)^{q}} \prod_{k=1}^{q} \sigma_{u_{k}, v_{k}} \sigma_{u_{k+1}, v_{k}} \prod_{(i, j) \in\left[p_{1}\right] \times\left[p_{2}\right]} \mathbb{E} G_{i j}^{\alpha_{i j}(\mathbf{c})}\left(G_{i j}^{2}-1\right)^{\beta_{i j}(\mathbf{c})},
\end{aligned}
$$

where $\left(\left[p_{1}\right] \times\left[p_{2}\right]\right)^{q}$ is the set of all cycles of length $2 q$ on a $p_{1}$-by- $p_{2}$ complete bipartite graph, $G_{i j}=Z_{i j} / \sigma_{i j}$ are i.i.d. standard normal distributed, and $\alpha_{i j}(\mathbf{c}), \beta_{i j}(\mathbf{c})$ are some graphical characteristic quantities of cycle $\mathbf{c}$ to be defined later. By gathering the cycles with the same "shape" s, we can show:

$$
\begin{aligned}
\mathbb{E} t r\left\{\left(Z Z^{\top}-\mathbb{E} Z Z^{\top}\right)^{q}\right\} \leq & \sum_{\mathbf{s}} \prod_{\alpha, \beta \geq 0}\left\{\mathbb{E} G^{\alpha}\left(G^{2}-1\right)^{\beta}\right\}^{m_{\alpha, \beta}(\mathbf{s})} \\
& \cdot\left\{p_{1} \sigma_{C}^{2\left(m_{L}(\mathbf{s})-1\right)} \sigma_{R}^{2 m_{R}(\mathbf{s})}\right\} \wedge\left\{p_{2} \sigma_{C}^{2 m_{L}(\mathbf{s})} \sigma_{R}^{2\left(m_{R}(\mathbf{s})-1\right)}\right\},
\end{aligned}
$$

where $m_{\alpha, \beta}(\mathbf{s}), m_{L}(\mathbf{s})$ and $m_{R}(\mathbf{s})$ are some graphical properties of the cycles with shape s to be defined later and $G \sim N(0,1)$. Meanwhile, we can develop a lower bound for the moment of standard Wishart matrix:

$$
\begin{aligned}
\mathbb{E} \operatorname{tr}\left(\left(H H^{\top}-\mathbb{E} H H^{\top}\right)^{q}\right) \geq & \sum_{\mathbf{s}} \prod_{\alpha, \beta \geq 0} \mathbb{E}\left\{G^{\alpha}\left(G^{2}-1\right)^{\beta}\right\}^{m_{\alpha, \beta}(\mathbf{s})} \\
& \cdot\left\{m_{1} \sigma_{C}^{2 m_{L}(\mathbf{s})-2} \cdot \sigma_{R}^{2 m_{R}(\mathbf{s})}\right\} \vee\left\{m_{2} \sigma_{C}^{2 m_{L}(\mathbf{s})} \cdot \sigma_{R}^{2 m_{R}(\mathbf{s})-2}\right\} .
\end{aligned}
$$

Lemma 2.4 follows by combining (2.7) and (2.8).

Next, Lemma 2.6 gives an upper bound on the moment of the standard Wishart matrix. The complete proof is provided in Section 5.1.

Lemma 2.6. Suppose $H \in \mathbb{R}^{m_{1} \times m_{2}}$ has i.i.d. standard Gaussian entries. Then for any integer $q \geq 2$,

$$
\begin{aligned}
&\left(\mathbb{E}\left\|H H^{\top}-\mathbb{E} H H^{\top}\right\|^{q}\right)^{1 / q} \leq 2 \sqrt{m_{1} m_{2}}+m_{1}+4\left(\sqrt{m_{1}}+\sqrt{m_{2}}\right) \sqrt{q}+2 q, \\
&\left(\mathbb{E} \operatorname{tr}\left\{\left(H H^{\top}-\mathbb{E} H H^{\top}\right)^{q}\right\}\right)^{1 / q} \leq 2^{1 / q}\left(m_{1} \wedge m_{2}\right)^{1 / q} \\
& \cdot\left(2 \sqrt{m_{1} m_{2}}+m_{1}+4\left(\sqrt{m_{1}}+\sqrt{m_{2}}\right) \sqrt{q}+2 q\right) .
\end{aligned}
$$


Remark 2.7 (Proof idea of Lemma 2.6). Let $\sigma_{i}(H)$ be the $i$-th singular value of $H$. The proof of Lemma 2.6 utilizes the following fact:

$$
\left\|H H^{\top}-\mathbb{E} H H^{\top}\right\|=\left\|H H^{\top}-m_{2} I_{m_{1}}\right\|=\max \left\{\sigma_{1}^{2}(H)-m_{2}, m_{2}-\sigma_{m_{1}}^{2}(H)\right\}
$$

and the concentration inequalities of the largest and smallest singular values of the Gaussian ensemble (e.g., [31]). See Section 5.1 for the complete proof.

Now, we are in position to finish the proof of Theorem 2.1.

Proof of Theorem 2.1. Without loss of generality, we assume $\sigma_{*}^{2}=\max _{i j} \sigma_{i j}^{2}=1$. Let $m_{1}=\left\lceil\sigma_{C}^{2}\right\rceil+2 q-1, m_{2}=\left\lceil\sigma_{R}^{2}\right\rceil+2 q-1$ for some $q$ to be specified later and $H$ be an $m_{1}$-by- $m_{2}$ random matrix with i.i.d. standard Gaussian entries. Lemmas 2.4 and 2.6 imply

$$
\begin{gathered}
\mathbb{E}\left\|Z Z^{\top}-\mathbb{E} Z Z^{\top}\right\| \leq\left(\mathbb{E} \operatorname{tr}\left\{\left(Z Z^{\top}-\mathbb{E} Z Z^{\top}\right)^{2 q}\right\}\right)^{1 / 2 q} \\
\stackrel{\text { Lemma } 2.4}{\leq}\left\{\left(\frac{p_{1}}{m_{1}} \wedge \frac{p_{2}}{m_{2}}\right) \cdot \mathbb{E} \operatorname{tr}\left\{H H^{\top}-\mathbb{E} H H^{\top}\right\}^{2 q}\right\}^{1 / 2 q} \\
\underset{\leq}{\leq} 2^{1 / 2 q}\left(\left(\frac{p_{1}}{m_{1}} \wedge \frac{p_{2}}{m_{2}}\right) m_{1} \wedge m_{2}\right)^{1 / 2 q}\left(2 \sqrt{m_{1} m_{2}}+m_{1}+4\left(\sqrt{m_{1}}+\sqrt{m_{2}}\right) \sqrt{2 q}+4 q\right) \\
\leq 2^{1 / 2 q}\left(p_{1} \wedge p_{2}\right)^{1 / 2 q}\left(2 \sigma_{C} \sigma_{R}+\sigma_{C}^{2}+10 \sigma_{C} \sqrt{q}+10 \sigma_{R} \sqrt{q}+24 q\right) .
\end{gathered}
$$

Let $q=K\left\lceil\log \left(p_{1} \wedge p_{2}\right)\right\rceil$ for $K=\left\lceil\frac{1}{\log \left(1+\varepsilon_{1}\right)}\right\rceil$, then we have

$$
\begin{aligned}
& \mathbb{E}\left\|Z Z^{\top}-\mathbb{E} Z Z^{\top}\right\| \\
\leq & 2^{1 / 2 K}\left(e^{q / K}\right)^{1 / 2 q}\left(2 \sigma_{C} \sigma_{R}+\sigma_{C}^{2}+10 \sigma_{C} \sqrt{q}+10 \sigma_{R} \sqrt{q}+24 q\right) \\
\leq & (2 e)^{1 / 2 K}\left(2 \sigma_{C} \sigma_{R}+\left(1+\epsilon_{2}\right) \sigma_{C}^{2}+10 \sqrt{K} \sigma_{R} \sqrt{\log \left(p_{1} \wedge p_{2}\right)}+\left(\frac{25}{\epsilon_{2}}+24\right) K \log \left(p_{1} \wedge p_{2}\right)\right) \\
\leq & 2\left(1+\epsilon_{1}\right) \sigma_{C} \sigma_{R}+\left(1+\epsilon_{1}\right)\left(1+\epsilon_{2}\right) \sigma_{C}^{2}+C_{1}\left(\epsilon_{1}\right) \sigma_{R} \sqrt{\log \left(p_{1} \wedge p_{2}\right)}+C_{2}\left(\epsilon_{1}, \epsilon_{2}\right) \log \left(p_{1} \wedge p_{2}\right) .
\end{aligned}
$$

Here,

$$
\begin{aligned}
C_{1}\left(\epsilon_{1}\right) & =10\left(1+\varepsilon_{1}\right) \sqrt{\left\lceil 1 / \log \left(1+\varepsilon_{1}\right)\right\rceil}, \\
C_{2}\left(\epsilon_{1}, \epsilon_{2}\right) & =\left(1+\varepsilon_{1}\right)\left\lceil 1 / \log \left(1+\varepsilon_{1}\right)\right\rceil\left(\frac{25}{\epsilon_{2}}+24\right) .
\end{aligned}
$$

\subsection{Lower bounds}

To show the tightness of the upper bound given earlier, we also develop the following minimax lower bound for the heteroskedastic Wishart-type concentration.

Theorem 2.8 (Lower bound of heteroskedastic Wishart-type concentration). Suppose $p_{1}, p_{2} \geq 4$. Consider the following set of $p_{1}$-by- $p_{2}$ random matrices,

$$
\mathcal{F}_{p}\left(\sigma_{*}, \sigma_{C}, \sigma_{R}\right)=\left\{Z \in \mathbb{R}^{p_{1} \times p_{2}}: \quad \begin{array}{l}
Z_{i j} \stackrel{i n d}{\sim} N\left(0, \sigma_{i j}^{2}\right), p=p_{1} \wedge p_{2}, \max _{i, j} \sigma_{i j} \leq \sigma_{*}, \\
\max _{i} \sum_{j=1}^{p_{2}} \sigma_{i j}^{2} \leq \sigma_{R}^{2}, \max _{j} \sum_{i=1}^{p_{1}} \sigma_{i j}^{2} \leq \sigma_{C}^{2}
\end{array}\right\} .
$$

For any $\left(\sigma_{*}, \sigma_{R}, \sigma_{C}\right)$ tuple satisfying $\min \left\{\sigma_{C}, \sigma_{R}\right\} \geq \sigma_{*} \geq \max \left\{\sigma_{C} / \sqrt{p_{1}}, \sigma_{R} / \sqrt{p_{2}}\right\}$, there exists a random Gaussian matrix $Z \in \mathcal{F}_{p}\left(\sigma_{*}, \sigma_{R}, \sigma_{C}\right)$ such that

$$
\mathbb{E}\left\|Z Z^{\top}-\mathbb{E} Z Z^{\top}\right\| \gtrsim \sigma_{C}^{2}+\sigma_{C} \sigma_{R}+\sigma_{R} \sigma_{*} \sqrt{\log p}+\sigma_{*}^{2} \log p
$$


The proof of Theorem 2.8 is given in Section 5.1.

Remark 2.9. Theorems 2.1 and 2.8 together establish the minimax optimal rate of $\mathbb{E}\left\|Z Z^{\top}-\mathbb{E} Z Z^{\top}\right\|$ in the class of $\mathcal{F}_{p}\left(\sigma_{*}, \sigma_{C}, \sigma_{R}\right)$. In other words, Theorem 2.8 shows that (2.1) yields the best upper bound for heteroskedastic Wishart-type concentration among all the bounds characterized by $\sigma_{C}, \sigma_{R}, \sigma_{*}$. We shall point out that the upper bound of Theorem 2.1 may not be tight for some specific values of $\left\{\sigma_{i j}^{2}\right\}$. For example, in Sections 3.3 and 3.4, we develop sharper bounds via a more refined analysis when the Wishart matrix has near-homoskedastic rows or columns.

Generally speaking, it remains an open problem to develop a heteroskedastic Wisharttype concentration inequality that is tight for all specific values of $\left\{\sigma_{i j}^{2}\right\}$. We leave this problem as future work.

\section{Extensions}

We consider several extensions of Theorem 2.1 in this section.

\subsection{Wishart-type concentration of non-Gaussian random matrices}

In this section, we generalize the developed concentration inequality for heteroskedastic Wishart matrices with more general entrywise distributions, such as sub-Gaussian, sub-exponential, heavy tailed, and bounded distributions. We first introduce the following lemma as a sub-Gaussian analog of Lemma 2.4.

Lemma 3.1 (Sub-Gaussian comparison). Suppose $Z \in \mathbb{R}^{p_{1} \times p_{2}}$ has independent meanzero symmetric sub-Gaussian entries:

$$
\mathbb{E} Z_{i j}=0, \quad \operatorname{Var}\left(Z_{i j}\right)=\sigma_{i j}^{2}, \quad\left\|Z_{i j} / \sigma_{i j}\right\|_{\psi_{2}} \leq \kappa .
$$

$M \in \mathbb{R}^{m_{1} \times m_{2}}$ has i.i.d. standard Gaussian entries. When $q \geq 1, m_{1}=\left\lceil\sigma_{C}^{2}\right\rceil+q-1$, $m_{2}=\left\lceil\sigma_{R}^{2}\right\rceil+q-1$, we have

$$
\mathbb{E} \operatorname{tr}\left\{\left(Z Z^{\top}-\mathbb{E} Z Z^{\top}\right)^{q}\right\} \leq(C \kappa)^{2 q}\left(\frac{p_{1}}{m_{1}} \wedge \frac{p_{2}}{m_{2}}\right) \mathbb{E} \operatorname{tr}\left\{\left(H H^{\top}-\mathbb{E} H H^{\top}\right)^{q}\right\} .
$$

The proof of Lemma 3.1 is deferred to Section 5.2.

Remark 3.2 (Proof ideas of Lemma 3.1). Compared to the proof of Lemma 2.4, the proof of Lemma 3.1 requires more delicate scheme to bound $\mathbb{E} G_{i j}^{\alpha_{i j}(\mathbf{c})}\left(G_{i j}^{2}-1\right)^{\beta_{i j}(\mathbf{c})}$ for non-standard-Gaussian distributed $G_{i j}:=Z_{i j} / \sigma_{i j}$. To this end, we introduce Lemma 5.2 to bound $\mathbb{E} G_{i j}^{\alpha_{i j}(\mathbf{c})}\left(G_{i j}^{2}-1\right)^{\beta_{i j}(\mathbf{c})}$ by a Gaussian analog:

$$
\mathbb{E} G_{i j}^{\alpha_{i j}(\mathbf{c})}\left(G_{i j}^{2}-1\right)^{\beta_{i j}(\mathbf{c})} \leq(C \kappa)^{2 q} \mathbb{E} G^{\alpha_{i j}(\mathbf{c})}\left(G^{2}-1\right)^{\beta_{i j}(\mathbf{c})}, \quad G \sim N(0,1) .
$$

As a consequence of Lemma 3.1, we have the following Wishart-type Concentration of sub-Gaussian random matrix.

Corollary 3.3 (Wishart-type concentration of sub-Gaussian random matrix). Suppose $Z \in \mathbb{R}^{p_{1} \times p_{2}}$ has independent mean-zero sub-Gaussian entries that satisfy (3.1). Then

$$
\mathbb{E}\left\|Z Z^{\top}-\mathbb{E} Z Z^{\top}\right\| \lesssim \kappa^{2}\left(\sigma_{C} \sigma_{R}+\sigma_{C}^{2}+\sigma_{R} \sigma_{*} \sqrt{\log \left(p_{1} \wedge p_{2}\right)}+\sigma_{*}^{2} \log \left(p_{1} \wedge p_{2}\right)\right) .
$$

Proof of Corollary 3.3. When all $Z_{i j}$ 's are symmetrically distributed, Corollary 3.3 follows from the proof of Theorem 2.1 along with Lemmas 2.6 and 3.1. If $Z_{i j}$ 's are not all symmetric, let $Z^{\prime}$ be an independent copy of $Z$, then each entry of $Z-Z^{\prime}$ has independent 
symmetric sub-Gaussian distribution. By Jensen's inequality, we have

$$
\begin{aligned}
\mathbb{E}\left\|Z Z^{\top}-\mathbb{E} Z Z^{\top}\right\| & =\mathbb{E}\left\|Z Z^{\top}+\mathbb{E}^{\prime} Z^{\prime}\left(Z^{\prime}\right)^{\top}-Z\left(\mathbb{E}^{\prime} Z^{\prime}\right)^{\top}-\left(\mathbb{E}^{\prime} Z^{\prime}\right) Z^{\top}-2 \mathbb{E} Z Z^{\top}\right\| \\
& =\mathbb{E}\left\|\mathbb{E}\left\{Z Z^{\top}+Z^{\prime}\left(Z^{\prime}\right)^{\top}-Z\left(Z^{\prime}\right)^{\top}-\left(Z^{\prime}\right) Z^{\top}-2 \mathbb{E} Z Z^{\top} \mid Z\right\}\right\| \\
& \leq \mathbb{E}\left[\mathbb{E}\left\{\left\|Z Z^{\top}+Z^{\prime}\left(Z^{\prime}\right)^{\top}-Z\left(Z^{\prime}\right)^{\top}-\left(Z^{\prime}\right) Z^{\top}-2 \mathbb{E} Z Z^{\top}\right\| \mid Z\right\}\right] \\
& =\mathbb{E}\left[\mathbb{E}^{\prime}\left\|\left(Z-Z^{\prime}\right)\left(Z-Z^{\prime}\right)^{\top}-\mathbb{E}\left(Z-Z^{\prime}\right)\left(Z-Z^{\prime}\right)^{\top}\right\|\right] \\
& \lesssim \kappa^{2}\left(\sigma_{C} \sigma_{R}+\sigma_{C}^{2}+\sigma_{R} \sigma_{*} \sqrt{\log \left(p_{1} \wedge p_{2}\right)}+\sigma_{*}^{2} \log \left(p_{1} \wedge p_{2}\right)\right) .
\end{aligned}
$$

Next, we turn to the Wishart-type concentration for random matrix $Z$ with heavytailed entries.

Theorem 3.4 (Wishart-type concentration for heavy-tailed random matrix). Suppose $\alpha \leq 1, Z \in \mathbb{R}^{p_{1} \times p_{2}}$ has independent entries, $\operatorname{Var}\left(Z_{i j}\right) \leq \sigma_{i j}^{2}$, and $\left\|Z_{i j} / \sigma_{i j}\right\|_{\psi_{\alpha}} \leq \kappa$ for all $i, j$. Given $\sigma_{C}, \sigma_{R}$, and $\sigma_{*}$ defined in (1.2), we have

$$
\mathbb{E}\left\|Z Z^{\top}-\mathbb{E} Z Z^{\top}\right\| \lesssim\left(\sigma_{C}+\sigma_{R}+\sigma_{*}\left(\log \left(p_{1} \wedge p_{2}\right)\right)^{1 / 2}\left(\log \left(p_{1} \vee p_{2}\right)\right)^{1 / \alpha-1 / 2}\right)^{2}-\sigma_{R}^{2}
$$

In a variety of applications, the observations and random perturbations are naturally bounded (e.g., adjacency matrix in network analysis [24] and single-nucleotide polymorphisms (SNPs) data in genomics [27]). Thus, we provide a Wishart-type concentration for entrywise uniformly bounded random matrices as follows.

Theorem 3.5 (Wishart-type concentration of bounded random Matrix). Suppose $Z \in$ $\mathbb{R}^{p_{1} \times p_{2}}, \mathbb{E} Z_{i j}=0, \operatorname{Var}\left(Z_{i j}\right)=\sigma_{i j}^{2},\left|Z_{i j}\right| \leq B$ almost surely, then

$$
\begin{aligned}
& \mathbb{E}\left\|Z Z^{\top}-\mathbb{E} Z Z^{\top}\right\| \\
\leq & \left(1+\epsilon_{1}\right)\left\{2 \sigma_{C} \sigma_{R}+\left(1+\epsilon_{2}\right) \sigma_{C}^{2}+C_{1}\left(\epsilon_{1}\right) B \sigma_{R} \sqrt{\log \left(p_{1} \wedge p_{2}\right)}+C_{2}\left(\epsilon_{1}, \epsilon_{2}\right) B^{2} \log \left(p_{1} \wedge p_{2}\right)\right\},
\end{aligned}
$$

where $C_{1}\left(\epsilon_{1}\right)$ and $C_{2}\left(\epsilon_{1}, \epsilon_{2}\right)$ are defined as in Theorem 2.1. If we further have $\max _{i, j} \sigma_{i j} \leq$ $\sigma_{*}$ and $B\left(\log \left(p_{1} \wedge p_{2}\right) / p_{1}\right)^{1 / 2} \ll \sigma_{*}$ for some $\sigma_{*}$, then

$$
\mathbb{E}\left\|Z Z^{\top}-\mathbb{E} Z Z^{\top}\right\| \leq(1+\epsilon)\left(2 \sqrt{p_{1} p_{2}}+p_{1}\right) \sigma_{*}^{2} .
$$

An immediate application of the previous theorem is the following Wishart-type concentration for independent Bernoulli random matrices.

Corollary 3.6 (Wishart-type Concentration of Bernoulli Random Matrix). Suppose $Z \in$ $\mathbb{R}^{p_{1} \times p_{2}}, A_{i j} \stackrel{i n d}{\sim} \operatorname{Bernoulli}\left(\theta_{i j}\right), \theta_{i j} \leq \theta_{*}$ and $\theta_{*} \geq C \log \left(p_{1} \wedge p_{2}\right) / p_{1}$. Then,

$$
\mathbb{E}\left\|(A-\Theta)(A-\Theta)^{\top}-\mathbb{E}(A-\Theta)(A-\Theta)^{\top}\right\| \lesssim\left(\sqrt{p_{1} p_{2}}+p_{1}\right) \theta_{*} .
$$

To prove Theorems 3.4 and 3.5, we establish the corresponding comparison lemmas for random matrices with heavy tail/bounded distributions, which is more technically involved from Gaussian/sub-Gaussian distributions due to the essential difference. The proofs of Theorems 3.4 and 3.5 are provided in Section 5.2.

Remark 3.7. It is helpful to summarize the heteroskedastic Wishart-type concentration inequalities with Gaussian, sub-Gaussian, heavy-tail, and bounded entries in a unified form:

$$
\mathbb{E}\left\|Z Z^{\top}-\mathbb{E} Z Z^{\top}\right\| \leq C_{0}\left\{\left(\sigma_{C}+\sigma_{R}+K\right)^{2}-\sigma_{R}^{2}\right\},
$$

where $K=\sigma_{*}\left(\log \left(p_{1} \wedge p_{2}\right)\right)^{1 / 2}$ and $C_{0}>1$ is a constant if the entries of $Z$ are subGaussian; $K=\sigma_{*}\left(\log \left(p_{1} \wedge p_{2}\right)\right)^{1 / 2}\left(\log \left(p_{1} \vee p_{2}\right)\right)^{1 / \alpha-1 / 2}$ and $C_{0}>1$ is a constant if $Z$ has bounded $\psi_{\alpha}$ norm; $K=C \sqrt{\log \left(p_{1} \wedge p_{2}\right)}$ and $C_{0}=1+\varepsilon$ if the entries of $Z$ are bounded; and $K=C \sigma_{*}\left(\log \left(p_{1} \wedge p_{2}\right)\right)^{1 / 2}$ and $C_{0}=(1+\varepsilon)$ if the entries of $Z$ are Gaussian. 


\subsection{Moments and tail bounds}

We study the general $b$-th moment and the tail probability of heteroskedastic Wisharttype matrix in the following theorem.

Theorem 3.8 (High-order moments and tail probability bounds). Suppose the conditions in Theorem 2.1 hold. For any $b>0$, we have

$$
\left\{\mathbb{E}\left\|Z Z^{\top}-\mathbb{E} Z Z^{\top}\right\|^{b}\right\}^{1 / b} \lesssim\left(\sigma_{C}+\sigma_{R}+\sigma_{*} \sqrt{b \vee \log \left(p_{1} \wedge p_{2}\right)}\right)^{2}-\sigma_{C}^{2}
$$

There exists uniform constant $C>0$ such that for any $x>0$,

$$
\mathbb{P}\left\{\left\|Z Z^{\top}-\mathbb{E} Z Z^{\top}\right\| \geq C\left(\left(\sigma_{C}+\sigma_{R}+\sigma_{*} \sqrt{\log \left(p_{1} \wedge p_{2}\right)}+x\right)^{2}-\sigma_{C}^{2}\right)\right\} \leq \exp \left(-x^{2}\right) .
$$

Since neither $\left\|Z Z^{\top}-\mathbb{E} Z Z^{\top}\right\|$ nor $\left\|Z Z^{\top}-\mathbb{E} Z Z^{\top}\right\|^{1 / 2}$ are Lipschitz continuous in $Z$, the classic Talagrand's concentration inequality [10, Theorem 6.10] does not directly apply to give the tail probability bound of $\left\|Z Z^{\top}-\mathbb{E} Z Z^{\top}\right\|$. We instead prove (3.7) via a more direct moment method. The complete proof is given in Section 5.3.

\subsection{Wishart matrix with near-homoskedastic rows}

In this section, we consider a special class of heteroskedastic matrices. Let $Z \in$ $\mathbb{R}^{p_{1} \times p_{2}}$ be a random matrix with independent, sub-Gaussian, and zero-mean entries. Suppose all entries in the same row of $Z$ share similar variance (i.e., there exists $\sigma_{i}^{2}$ such that $\sigma_{i j}$ approximately equals $\sigma_{i}^{2}$ for all $i, j$ ). Then the $p_{2}$ columns of $Z$, i.e., $\{Z \cdot j\}_{j=1}^{p_{2}}$, have approximately equal covariance matrix, $\operatorname{diag}\left(\sigma_{1}^{2}, \ldots, \sigma_{p_{1}}^{2}\right)$. In this case, $\frac{1}{n} Z Z^{\top}=\frac{1}{n} \sum_{j=1}^{n} Z_{\cdot j} Z_{\cdot j}^{\top}$ is the sample covariance matrix. It is of great interest to analyze $\left\|Z Z^{\top}-\mathbb{E} Z Z^{\top}\right\|$, i.e., the concentration of the sample covariance matrix in both probability and statistics $[3,12]$.

Note that Corollary 3.3 directly implies

$$
\mathbb{E}\left\|Z Z^{\top}-\mathbb{E} Z Z^{\top}\right\| \lesssim \sum_{i} \sigma_{i}^{2}+\sqrt{p_{2} \sum_{i} \sigma_{i}^{2}} \cdot \max _{i} \sigma_{i}+\sqrt{p_{2} \log \left(p_{1} \wedge p_{2}\right)} \max _{i} \sigma_{i}^{2} .
$$

With a more careful analysis, we can derive a better concentration inequality than (3.8) without the logarithmic terms.

Theorem 3.9. Suppose $Z$ is a $p_{1}$-by- $p_{2}$ random matrix with independent mean-zero subGaussian entries. If there exist $\sigma_{1}, \ldots, \sigma_{p} \geq 0$ such that $\left\|Z_{i j} / \sigma_{i}\right\|_{\psi_{2}} \leq C_{K}$ for constant $C_{K}>0$, then

$$
\mathbb{E}\left\|Z Z^{\top}-\mathbb{E} Z Z^{\top}\right\| \lesssim \sum_{i} \sigma_{i}^{2}+\sqrt{p_{2} \sum_{i} \sigma_{i}^{2}} \cdot \max _{i} \sigma_{i} .
$$

Remark 3.10. We also note that a similar result of Theorem 3.9 can be derived from Koltchinskii and Lounici [18]. Their result is based on generic chaining argument with the assumption that all columns of $Z$ are i.i.d. Here, we assume independence and an upper bound on the Orlicz- $\psi_{2}$ norm of each entry, while allow the distributions to be non-identical.

The following theorem gives a lower bound on the concentration of Wishart matrix with homoskedastic rows.

Theorem 3.11. If $Z \in \mathbb{R}^{p_{1} \times p_{2}}, Z_{i j} \stackrel{i n d}{\sim} N\left(0, \sigma_{i}^{2}\right)$, we have

$$
\mathbb{E}\left\|Z Z^{\top}-\mathbb{E} Z Z^{\top}\right\| \gtrsim \sum_{i} \sigma_{i}^{2}+\sqrt{p_{2} \sum_{i} \sigma_{i}^{2}} \cdot \max _{i} \sigma_{i} .
$$


The proof of Theorem 3.11 is deferred to Section 5.4. Theorems 3.9 and 3.11 render an exact rate of Wishart-type concentration for random matrices with homoskedastic rows:

$$
\mathbb{E}\left\|Z Z^{\top}-\mathbb{E} Z Z^{\top}\right\| \asymp \sum_{i} \sigma_{i}^{2}+\sqrt{p_{2} \sum_{i} \sigma_{i}^{2}} \max _{i} \sigma_{i}, \quad \text { if } \operatorname{Var}\left(Z_{i j}\right) \stackrel{i n d}{\sim} N\left(0, \sigma_{i}^{2}\right) .
$$

The rest of this section is dedicated to the proof of Theorems 3.9. We only prove for Gaussian Wishart-type random matrices since the sub-Gaussian case follows similarly. We first introduce a key tool to sequentially reduce the number of rows of $Z$. The tool, as summarized in the following lemma, may of independent interest.

Lemma 3.12 (Variance contraction inequality of Gaussian random matrix). Suppose $G \in \mathbb{R}^{p_{1} \times p_{2}}$ and $\tilde{G} \in \mathbb{R}^{\left(p_{1}-1\right) \times p_{2}}$ are two random matrices with independent Gaussian entries satisfying

$$
\mathbb{E} G_{i j}=\mathbb{E} \tilde{G}_{i j}=0, \quad \operatorname{Var}\left(G_{i j}\right)=\sigma_{i j}^{2}, \quad \operatorname{Var}\left(\tilde{G}_{i j}\right)= \begin{cases}\sigma_{i j}^{2}, & 1 \leq i \leq p_{1}-2 \\ \sigma_{p_{1}-1, j}^{2}+\sigma_{p_{1}, j}^{2}, & i=p_{1}-1 .\end{cases}
$$

In other words, $G$ and $\tilde{G}$ are identical distributed in their first $\left(p_{1}-2\right)$ rows; the variance of the last row of $\tilde{G}$ is the sum of last two rows' variances of $G$. Then for any positive integer $q$,

$$
\operatorname{tr}\left(\left(G G^{\top}-\mathbb{E} G G^{\top}\right)^{q}\right) \leq \operatorname{tr}\left(\left(\tilde{G} \tilde{G}^{\top}-\mathbb{E} \tilde{G} \tilde{G}^{\top}\right)^{q}\right) .
$$

The proof of Lemma 3.12 is provided in Section 5.4. Now we are ready to prove Theorem 3.9.

Proof of Theorem 3.9. Denote $\sigma_{C}^{2}=\sum_{i} \sigma_{i}^{2}, \sigma_{*}=\max _{i} \sigma_{i}$. Assume $\sigma_{*}=1$ without loss of generality. Set $q=2\left\lceil\sigma_{C}^{2}\right\rceil$. We use mathematical induction on $p_{1}$ to show the following upper bound: for some uniform constant $C>0$ (which does not dependent on $p_{1}, p_{2}, \sigma_{C}$ ), we have

$$
\left(\mathbb{E} \operatorname{tr}\left\{\left(Z Z^{\top}-\mathbb{E} Z Z^{\top}\right)^{q}\right\}\right)^{1 / q} \leq C\left(\sigma_{C}^{2}+\sqrt{p_{2}} \sigma_{C}\right)
$$

- If $p_{1} \leq 2 q$, Lemma 2.4 yields

$$
\mathbb{E} \operatorname{tr}\left\{\left(Z Z^{\top}-\mathbb{E} Z Z^{\top}\right)^{q}\right\} \leq\left(\frac{p_{1}}{m_{1}} \wedge \frac{p_{2}}{m_{2}}\right) \mathbb{E} \operatorname{tr}\left\{\left(H H^{\top}-\mathbb{E} H H^{\top}\right)^{q}\right\} .
$$

Here, $H$ is a $m_{1}$-by- $m_{2}$ dimensional matrix with i.i.d. standard Gaussian entries and

$$
m_{1}=\left\lceil\sigma_{C}^{2}\right\rceil+q-1=3\left\lceil\sigma_{C}^{2}\right\rceil-1, \quad m_{2}=p_{2}+2\left\lceil\sigma_{C}^{2}\right\rceil-1 .
$$

Additionally, by Lemma 2.6,

$$
\begin{aligned}
\left(\mathbb{E}\left\{\left(Z Z^{\top}-\mathbb{E} Z Z^{\top}\right)^{q}\right\}\right)^{1 / q} & \leq\left(\left(\frac{p_{1}}{m_{1}} \wedge \frac{p_{2}}{m_{2}}\right) \mathbb{E} \operatorname{tr}\left\{\left(H H^{\top}-\mathbb{E} H H^{\top}\right)^{q}\right\}\right)^{1 / q} \\
& \leq\left(\mathbb{E}\left(\frac{p_{1}}{m_{1}} \wedge \frac{p_{2}}{m_{2}}\right) m_{1}\left\|H H^{\top}-\mathbb{E} H H^{\top}\right\|^{q}\right)^{1 / q} \\
& \leq p_{1}^{1 / q}\left(2 \sqrt{m_{1} m_{2}}+m_{1}+4\left(\sqrt{m_{1}}+\sqrt{m_{2}}\right) \sqrt{q}+2 q\right) \\
& (3.11) \\
\leq & (2 q)^{1 / q} \cdot C\left(\sqrt{p_{2}} \sigma_{C}+\sigma_{C}^{2}\right) \\
& \leq C\left(\sqrt{p_{2}} \sigma_{C}+\sigma_{C}^{2}\right)
\end{aligned}
$$

which implies (3.10). 
- Suppose the statement (3.10) holds for $Z \in \mathbb{R}^{\left(p_{1}-1\right) \times p_{2}}$ for some $p_{1}>2 q$, we further consider the case where $Z \in \mathbb{R}^{p_{1} \times p_{2}}$. Note that

$$
1=\sigma_{*}^{2}=\sigma_{1}^{2} \geq \sigma_{2}^{2} \geq \cdots \geq \sigma_{p_{1}}^{2} \geq 0 .
$$

By such the ordering,

$$
\sigma_{p_{1}-1}^{2}+\sigma_{p_{1}}^{2} \leq \frac{2}{p_{1}} \sum_{i=1}^{p_{1}} \sigma_{i}^{2}=\frac{2}{p_{1}} \sigma_{C}^{2} \leq \frac{2 \sigma_{C}^{2}}{2 q} \leq \frac{2 \sigma_{C}^{2}}{4\left\lceil\sigma_{C}^{2}\right\rceil} \leq 1=\sigma_{*}^{2} .
$$

By Lemma 3.12, we have

$$
\operatorname{tr}\left(\left(Z Z^{\top}-\mathbb{E} Z Z^{\top}\right)^{q}\right) \leq \operatorname{tr}\left(\left(\tilde{Z} \tilde{Z}^{\top}-\mathbb{E} \tilde{Z} \tilde{Z}^{\top}\right)^{q}\right) .
$$

where $\tilde{Z}$ is a $\left(p_{1}-1\right)$-by- $p_{2}$ random matrix with independent entries and

$$
\mathbb{E}(\tilde{Z})=0, \quad \operatorname{Var}\left((\tilde{Z})_{i j}\right)= \begin{cases}\sigma_{i}^{2}, & \text { if } 1 \leq i \leq p_{1}-2 \\ \sigma_{p-1}^{2}+\sigma_{p}^{2}, & \text { if } 1 \leq i \leq p_{1}-1 .\end{cases}
$$

By (3.12), we have $\max _{i, j} \operatorname{Var}\left((\tilde{Z})_{i j}\right) \leq \sigma_{*}^{2}$. Meanwhile, $\sum_{i=1}^{p_{1}-1} \operatorname{Var}\left((\tilde{Z})_{i j}\right)=$ $\sum_{i=1}^{p_{1}} \sigma_{i}^{2}=\sigma_{C}^{2}$. Thus, the induction assumption of (3.10) implies

$$
\left(\mathbb{E}\left\{\left(Z Z^{\top}-\mathbb{E} Z Z^{\top}\right)^{q}\right\}\right)^{1 / q} \leq\left(\mathbb{E}\left\{\left(\tilde{Z} \tilde{Z}^{\top}-\mathbb{E} \tilde{Z} \tilde{Z}^{\top}\right)^{q}\right\}\right)^{1 / q} \leq C\left(\sqrt{p_{2}} \sigma_{C}+\sigma_{C}^{2}\right) .
$$

By induction, we have proved that (3.10) holds in general. Therefore,

$$
\mathbb{E}\left\|Z Z^{\top}-\mathbb{E} Z Z^{\top}\right\| \leq\left(\mathbb{E t r}\left\{\left(Z Z^{\top}-\mathbb{E} Z Z^{\top}\right)^{q}\right\}\right)^{1 / q} \lesssim \sqrt{p_{2}} \sigma_{C}+\sigma_{C}^{2} .
$$

\subsection{Wishart matrix with near-homoskedastic columns}

Let $Z \in \mathbb{R}^{p_{1} \times p_{2}}$ be a random matrix with independent entries. We consider another case of interest that all entries in each column of $Z$ have the similar variance (i.e., there exist $\sigma_{j}$ such that $\left.\sigma_{i j} \approx \sigma_{j}^{2}, \forall i, i^{\prime} \in\left[p_{1}\right], \forall j \in\left[p_{2}\right]\right)$. This model has been used to characterize heteroskedastic independent samples in statistical applications [17]. Applying Theorem 2.1, one obtains

$$
\mathbb{E}\left\|Z Z^{\top}-\mathbb{E} Z Z^{\top}\right\| \lesssim \sqrt{p_{1} \sum_{j} \sigma_{j}^{2}} \max _{j} \sigma_{j}+p_{1} \max _{j} \sigma_{j}^{2} .
$$

As the direct upper bound of (3.13) may be sub-optimal, we prove the following upper and lower bounds via a more careful analysis.

Theorem 3.13. Suppose $Z \in \mathbb{R}^{p_{1} \times p_{2}}$ has independent, mean-zero, and sub-Gaussian entries. Assume there exist $\sigma_{1}, \ldots, \sigma_{n} \geq 0$ such that $\left\|Z_{i j} / \sigma_{j}\right\|_{\psi_{2}} \leq C_{K}$ for constant $C_{K}>0$. Then,

$$
\mathbb{E}\left\|Z Z^{\top}-\mathbb{E} Z Z^{\top}\right\| \lesssim \sqrt{p_{1} \sum_{j} \sigma_{j}^{4}}+p_{1} \max _{j} \sigma_{j}^{2}
$$

Theorem 3.14. If $Z \in \mathbb{R}^{p_{1} \times p_{2}}, Z_{i j} \stackrel{\text { ind }}{\sim} N\left(0, \sigma_{j}^{2}\right)$, we have

$$
\mathbb{E}\left\|Z Z^{\top}-\mathbb{E} Z Z^{\top}\right\| \gtrsim \sqrt{p_{1} \sum_{j} \sigma_{j}^{4}}+p_{1} \max _{j} \sigma_{j}^{2} .
$$


The proof of Theorem 3.14 is deferred to Section 5.5. Now we consider the proof of Theorem 3.13. Since the Gaussian comparison lemma (Lemma 2.4) cannot give the desired term $\sum_{j=1}^{p_{2}} \sigma_{j}^{4}$, we turn to study the expansion of $\operatorname{Etr}\left\{\left(\Delta\left(Z Z^{\top}\right)\right)^{q}\right\}$, where $\Delta\left(Z Z^{\top}\right)$ equals to $Z Z^{\top}$ with all diagonal entries set to zero. The expansion of $\operatorname{Etr}\left\{\left(\Delta\left(Z Z^{\top}\right)\right)^{q}\right\}$ can be related to the cycles in a complete graph for which every edge is visited $\{0,4,8,12 \ldots\}$ times. Based on this new idea, we introduce the following lemma.

Lemma 3.15. Suppose $Z \in \mathbb{R}^{p_{1} \times p_{2}}, Z_{i j} \stackrel{i n d}{\sim} N\left(0, \sigma_{i j}^{2}\right)$, and $\sigma_{i j} \leq \sigma_{j}$. For a square matrix $A$, let $\Delta(A)$ be $A$ with all diagonal entries set to zero and $D(A)$ be $A$ with all off-diagonal entries set to zero. For any integer $q \geq 1$, suppose $H \in \mathbb{R}^{p_{1} \times m}$ have i.i.d. standard normal entries and $m=\left\lceil\sum_{j=1}^{p_{2}} \sigma_{j}^{4}\right\rceil+q-1$. Then,

$$
\operatorname{Etr}\left\{\left(\Delta\left(Z Z^{\top}\right)\right)^{q}\right\} \leq \mathbb{E} \operatorname{tr}\left\{\left(\Delta\left(H H^{\top}\right)\right)^{q}\right\}
$$

The proof of Lemma 3.15 is provided in Section 5.5. Next, we prove Theorem 3.13.

Proof of Theorem 3.13. Denote $\sigma_{R}^{2}=\sum_{j} \sigma_{j}^{2}, \sigma_{*}=\max _{i} \sigma_{i}$. Without loss of generality, we assume $\sigma_{*}=1$. Note that $\mathbb{E}\left\|Z Z^{\top}-\mathbb{E} Z Z^{\top}\right\| \leq \mathbb{E}\left\|D\left(Z Z^{\top}\right)-\mathbb{E} Z Z^{\top}\right\|+\mathbb{E}\left\|\Delta\left(Z Z^{\top}\right)\right\|$. It suffices to bound the two terms separately. Since $D\left(Z Z^{\top}\right)-\mathbb{E} Z Z^{\top}$ is a diagonal matrix with independent diagonal entries, we have

$$
\left\|D\left(Z Z^{\top}\right)-\mathbb{E} Z Z^{\top}\right\|=\max _{i \in\left[p_{1}\right]}\left|\sum_{j=1}^{p_{2}} Z_{i j}^{2}-\mathbb{E} \sum_{j=1}^{p_{2}} Z_{i j}^{2}\right| .
$$

With Bernstein inequality and union bound, we have

$$
\mathbb{P}\left(\max _{i \in\left[p_{1}\right]}\left|\sum_{j=1}^{p_{2}} Z_{i j}^{2}-\mathbb{E} \sum_{j=1}^{p_{2}} Z_{i j}^{2}\right|>t\right) \leq 2 \exp \left(\log p_{1}-c\left(\frac{t^{2}}{\sum_{j=1}^{p_{2}} \sigma_{j}^{4}} \wedge \frac{t}{\sigma_{*}^{2}}\right)\right) .
$$

Integration over the tail further yields

$$
\mathbb{E} \max _{i \in\left[p_{1}\right]}\left|\sum_{j=1}^{p_{2}} Z_{i j}^{2}-\mathbb{E} \sum_{j=1}^{p_{2}} Z_{i j}^{2}\right| \lesssim \sqrt{\log p_{1} \sum_{j=1}^{p_{2}} \sigma_{j}^{4}}+\sigma_{*}^{2} \log p_{1} .
$$

Next, we use moment method to bound $\mathbb{E}\left\|\Delta\left(Z Z^{\top}\right)\right\|$. For any even positive integer $q$, by Lemma 3.15,

$$
\mathbb{E}\left\|\Delta\left(Z Z^{\top}\right)\right\| \leq\left(\mathbb{E t r}\left\{\left(\Delta\left(Z Z^{\top}\right)\right)^{q}\right\}\right)^{1 / q} \leq\left(\mathbb{E t r}\left\{\left(\Delta\left(H H^{\top}\right)\right)^{q}\right\}\right)^{1 / q} .
$$

Here $H$ is a $p_{1}$-by- $m$ random matrix with i.i.d. $N(0,1)$ entries and $m=\left\lceil\sum_{j=1}^{p_{2}} \sigma_{j}^{4}\right\rceil+q-1$. Thus it suffices to bound $\left(\mathbb{E} \operatorname{tr}\left\{\left(\Delta\left(H H^{\top}\right)\right)^{q}\right\}\right)^{1 / q}$.

On the one hand, by Lemma 2.6, $\forall q \geq 2$,

$$
\left(\mathbb{E}\left\|H H^{\top}-\mathbb{E} H H^{\top}\right\|^{q}\right)^{1 / q} \leq 2 \sqrt{p_{1} m}+m+4\left(\sqrt{p_{1}}+\sqrt{m}\right) \sqrt{q}+2 q .
$$

On the other hand, note that $\left\|D\left(H H^{\top}\right)-\mathbb{E} H H^{\top}\right\|=\max _{i \in[m]}\left|X_{i}\right|$, where $X_{i}$ are independent centralized $\chi_{m}^{2}$ random variable. By the Chi-square concentration and union bound, we have

$$
\mathbb{P}\left(\max _{i \in\left[p_{1}\right]}\left|X_{i}\right|^{q}>t\right) \leq 2 \exp \left(\log p_{1}-c\left(\frac{t^{2 / q}}{m} \wedge t^{1 / q}\right)\right)
$$


Integration gives

$$
\mathbb{E} \max _{i \in\left[p_{1}\right]}\left|X_{i}\right|^{q} \leq C^{q}\left(\log ^{q} p_{1}+\left(\sqrt{m \log p_{1}}\right)^{q}\right) .
$$

Then it follows that

$$
\begin{gathered}
\left(\mathbb{E} \operatorname{tr}\left\{\left(\Delta\left(H H^{\top}\right)\right)^{q}\right\}\right)^{1 / q} \leq\left(p_{1} \mathbb{E}\left\|\Delta\left(H H^{\top}\right)\right\|^{q}\right)^{1 / q} \\
\leq p_{1}^{1 / q}\left(\mathbb{E}\left\|H H^{\top}-\mathbb{E} H H^{\top}\right\|^{q}\right)^{1 / q}+\left(\mathbb{E}\left\|D\left(H H^{\top}-\mathbb{E} H H^{\top}\right)\right\|^{q}\right)^{1 / q} \\
\stackrel{(3.18)(3.19)}{\lesssim} p_{1}^{1 / q} \cdot\left(\sqrt{p_{1} m}+p_{1}+4\left(\sqrt{p_{1}}+\sqrt{m}\right) \sqrt{q}+2 q\right) .
\end{gathered}
$$

Now we specify $q=2 p_{1}$ and get

$$
\mathbb{E}\left\|\Delta\left(Z Z^{\top}\right)\right\| \stackrel{(3.17)}{\leq}\left(\mathbb{E} \operatorname{tr}\left\{\left(\Delta\left(H H^{\top}\right)\right)^{q}\right\}\right)^{1 / q} \lesssim \sqrt{p_{1} \sum_{j=1}^{n} \sigma_{j}^{4}}+p_{1} .
$$

This together with (3.16) completes the proof of this theorem.

\section{Applications}

The concentration bounds established in the previous sections have a range of applications. In this section, we illustrate the usefulness of the heteroskedastic Wisharttype concentration by applications to low-rank matrix denoising and heteroskedastic clustering.

Consider the following "signal + noise" model:

$$
Y=X+Z
$$

where $X \in \mathbb{R}^{p_{1} \times p_{2}}$ is a (approximately) low-rank matrix of interest, $Z$ is the random noise with independent entries, and $Y$ is the observation. This model has attracted significant attention in probability and statistics [5, 7, 14, 26], and has also been the prototypical setting in various applications, such as bipartite stochastic block model [15], exponential family PCA [22], top- $k$ ranking from pairwise comparison [23]. In these applications, the leading singular values/vectors of $X$ often contain information of interest. A straightforward way to estimate the leading singular values/vectors of $X$ (which are also the square root eigenvalues and the eigenvectors of $\left.X X^{\top}\right)$ is by evaluating the spectrum of $Y$ (or equivalently $Y Y^{\top}$ ). Suppose $\lambda_{i}\left(Y Y^{\top}\right), \lambda_{i}\left(X X^{\top}\right), v_{i}\left(Y Y^{\top}\right), v_{i}\left(Y Y^{\top}\right)$ are the $i$ th eigenvalue and $i$ th eigenvector of $Y Y^{\top}, X X^{\top}$, respectively. The classic perturbation theory (e.g., Weyl [34] and David-Kahan [13]) yield the following sharp bounds,

$$
\begin{gathered}
\left|\lambda_{i}\left(Y Y^{\top}\right)-\lambda_{i}\left(X X^{\top}\right)\right| \leq\left\|Y Y^{\top}-X X^{\top}\right\| \\
\left\|v_{i}\left(Y Y^{\top}\right) \pm v_{i}\left(Y Y^{\top}\right)\right\|_{2} \lesssim \frac{\left\|Y Y^{\top}-X X^{\top}\right\|}{\min _{j=i, i+1}\left\{\lambda_{j-1}\left(X X^{\top}\right)-\lambda_{j}\left(X X^{\top}\right)\right\}} .
\end{gathered}
$$

Then, a tight upper bound for the perturbation $Y Y^{\top}-X X^{\top}$ is critical to quantify the estimation accuracy of $\lambda_{i}\left(Y Y^{\top}\right), v_{i}\left(Y Y^{\top}\right)$ to $\lambda_{i}\left(X X^{\top}\right), v_{i}\left(X X^{\top}\right)$. By expansion, the perturbation of $Y Y^{\top}-X X^{\top}$ can be written as

$$
Y Y^{\top}-X X^{\top}=X Z^{\top}+Z X^{\top}+\mathbb{E} Z Z^{\top}+\left(Z Z^{\top}-\mathbb{E} Z Z^{\top}\right)
$$

Here, $\mathbb{E} Z Z^{\top}$ is a deterministic diagonal matrix; $\left\|X Z^{\top}\right\|=\left\|Z X^{\top}\right\|$ are the spectral norm of a random matrix multiplied by a deterministic matrix, which has been considered in [32]; The term $\left\|Z Z^{\top}-Z Z^{\top}\right\|$ can often be the dominating and most complicated part in 
(4.1) and the heteroskedastic Wishart-type concentration inequality established in the present paper provides a powerful tool for analyzing it.

We further illustrate through a specific application to high-dimensional heteroskedastic clustering. The clustering is an ubiquitous task in statistics and machine learning [16]. Suppose we observe a two-component Gaussian mixture:

$$
Y_{j}=l_{j} \mu+\varepsilon_{j}, \quad \varepsilon_{j}=\left(\varepsilon_{1 j}, \ldots, \varepsilon_{p j}\right)^{\top}, \quad \varepsilon_{i j} \stackrel{i n d}{\sim} N\left(0, \sigma_{i}^{2}\right), \quad j=1, \ldots, n .
$$

Here, $\mu$ is an unknown deterministic vector in $\mathbb{R}^{p}$ and $l_{j} \in\{-1,1\}$ are unknown labels of two classes. While most existing works focus on the homoskedastic setting, we consider a heteroskedastic setting where the noise variance $\sigma_{i}^{2}$ may vary across different coordinates. Then, the sample $\left\{Y_{j}\right\}_{j=1}^{n}$ can be written in a matrix form, $Y=X+Z$, where

$$
Y=\left[Y_{1}^{\top}, Y_{2}^{\top}, \cdots, Y_{n}^{\top}\right]^{\top}, \quad X=\left[l_{1}, l_{2}, \cdots, l_{n}\right]^{\top} \mu, \quad \text { and } \quad Z=\left(\varepsilon_{i j}\right) .
$$

Our goal is to cluster $\left\{Y_{j}\right\}_{j=1}^{n}$ into two groups, or equivalently to estimate the hidden label $\left\{l_{j}\right\}_{j=1}^{n}$. Let $\hat{v}$ be the first eigenvector of $Y Y^{\top}$. As $\hat{v}$ is an estimation of $l$, it is straightforward to cluster as

$$
\hat{l}_{j}=\operatorname{sgn}\left(\hat{v}_{j}\right), \quad j=1, \ldots, n \text {. }
$$

Applying Theorem 3.13 and perturbation bound of $\left\|X Z^{\top}\right\|[36$, Lemma 3] on (4.1), it can be shown that

$$
\mathbb{E}\left\|Y Y^{\top}-\mathbb{E} Z Z^{\top}-X X^{\top}\right\| \lesssim n\|\mu\| \sigma_{*}+n \sigma_{*}^{2}+\sqrt{n \sum_{j} \sigma_{j}^{4}} .
$$

Combining this with the Davis-Kahan Theorem [13], we obtain the following result.

Theorem 4.1. Let $\sigma_{*}=\max _{i} \sigma_{i}$ and $\tilde{\sigma}=\left(\sum_{i} \sigma_{i}^{4}\right)^{1 / 4}$. The estimator in (4.3) satisfies

$$
\operatorname{EM}(l, \hat{l}) \lesssim \frac{n\|\mu\|_{2} \sigma_{*}+n \sigma_{*}^{2}+\sqrt{n} \tilde{\sigma}^{2}}{n\|\mu\|_{2}^{2}} \wedge 1
$$

Here, $\mathcal{M}(l, \hat{l})$ is the misclassification rate defined as

$$
\mathcal{M}(l, \hat{l})=\frac{1}{n} \min \left\{\sum_{i=1}^{n} 1_{\left\{l_{i} \neq \hat{l}_{i}\right\}}, \sum_{i=1}^{n} 1_{\left\{l_{i} \neq-\hat{l}_{i}\right\}}\right\} .
$$

The complete proof of Theorem 4.1 is deferred to Section 5.6. By (4.4), the clustering is consistent (i.e., $\mathbb{E} \mathcal{M}(l, \hat{l})=o(1)$ ) as long as

$$
\|\mu\|_{2} \gg \sigma_{*} \vee\left(\tilde{\sigma} / n^{1 / 4}\right) .
$$

The following lower bound shows that the signal-noise-ration condition (4.6) is necessary to ensure a consistent classification. The proof is provided in Section 5.6.

Theorem 4.2. Suppose $\sigma_{*} \leq \tilde{\sigma} \leq p^{1 / 4} \sigma_{*}$. Consider the following class of distributions on $\mathbb{R}^{n \times p}$ :

$$
\mathcal{P}_{l, \lambda}\left(\sigma_{*}, \tilde{\sigma}\right)=\left\{P_{Y}: Y=X+Z \in \mathbb{R}^{n \times p}: \begin{array}{l}
X=l \mu^{\top}, Z_{i j} \stackrel{i n d}{\sim} N\left(0, \sigma_{j}^{2}\right), \\
\|\mu\| \geq \lambda, \max _{j} \sigma_{j} \leq \sigma_{*}, \sum_{i=1}^{p} \sigma_{i}^{4} \leq \tilde{\sigma}^{4}
\end{array}\right\} .
$$

There exists a universal constant $c>0$, such that if $\lambda<c\left(\sigma_{*} \vee\left(\tilde{\sigma} / n^{1 / 4}\right)\right)$, we have

$$
\inf _{\hat{l}} \sup _{\mathcal{P}_{l, \lambda}\left(\sigma_{*}, \tilde{\sigma}\right)} \mathbb{E} \mathcal{M}(l, \hat{l}) \geq 1 / 4
$$




\section{Additional proofs}

\subsection{Proofs for main results}

In this section, we collect the proofs of upper and lower bound results in Section 2 including Lemma 2.4, Lemma 2.6, Proposition 2.3 and Theorem 4.2.

Proof of Lemma 2.4. This proof shares similarity but shows more distinct aspects, compared with the one of Wigner-type [4, Proposition 2.1]. We assume $\sigma_{*}=1$ throughout the proof without loss of generality. We divide the proof into two steps, which targets on the two sides of the inequalities, respectively.

Step 1. One can check that $\mathbb{E} Z Z^{\top}=\operatorname{diag}\left(\left\{\sum_{j=1}^{p_{2}} \sigma_{i j}^{2}\right\}_{i=1}^{p_{1}}\right)$. Consider the following expansion,

$$
\begin{aligned}
& \mathbb{E} \operatorname{tr}\left\{\left(Z Z^{\top}-\mathbb{E} Z Z^{\top}\right)^{q}\right\}=\sum_{u_{1}, \ldots, u_{q}, u_{q+1} \in\left[p_{1}\right]} \mathbb{E} \prod_{k=1}^{q}\left(Z Z^{\top}-\mathbb{E} Z Z^{\top}\right)_{u_{k}, u_{k+1}} \\
= & \sum_{\substack{u_{1}, \ldots, u_{q}, u_{q+1} \in\left[p_{1}\right] \\
k}} \mathbb{E} \prod_{k=1}^{q}\left\{\sum_{v_{k} \in\left[p_{2}\right]}\left(Z_{u_{k}, v_{k}} Z_{u_{k+1}, v_{k}}-1_{\left\{u_{k}=u_{k+1}\right\}} \mathbb{E} Z_{u_{k}, v_{k}}^{2}\right)\right\} \\
= & \sum_{\substack{u_{1}, \ldots, u_{q}, u_{q+1} \in\left[p_{1}\right] \\
v_{1}, \ldots, v_{q} \in\left[p_{2}\right]}} \mathbb{E} \prod_{k=1}^{q}\left(Z_{u_{k}, v_{k}} Z_{u_{k+1}, v_{k}}-\sigma_{u_{k}, v_{k}}^{2} \cdot 1_{\left\{u_{k}=u_{k+1}\right\}}\right) .
\end{aligned}
$$

Here, the indices are in module $q$, i.e., $u_{1}=u_{q+1}$. Next, we consider the bipartite graph from $\left[p_{1}\right]$ on $\left[p_{2}\right]$ and the cycles of length $2 q$, i.e., $\mathbf{c}:=\left(u_{1} \rightarrow v_{1} \rightarrow u_{2} \rightarrow v_{2} \rightarrow \ldots \rightarrow u_{q} \rightarrow\right.$ $\left.v_{q} \rightarrow u_{q+1}=u_{1}\right)$. For any $(i, j) \in\left[p_{1}\right] \times\left[p_{2}\right]$, let

$$
\begin{aligned}
& \alpha_{i j}(\mathbf{c})=\operatorname{Card}\left\{k:\left(u_{k}=i, v_{k}=j, u_{k+1} \neq i\right) \text { or }\left(u_{k} \neq i, v_{k}=j, u_{k+1}=i\right)\right\} ; \\
& \beta_{i j}(\mathbf{c})=\operatorname{Card}\left\{k: u_{k}=u_{k+1}=i, v_{k}=j\right\} .
\end{aligned}
$$

Then, $\alpha_{i j}(\mathcal{L})$ is the number of times that the edge $(i, j)$ is visited exactly once by sub-path $u_{k} \rightarrow v_{k} \rightarrow u_{k+1} ; \beta_{i j}(\mathbf{c})$ is the number of times that the edge $(i, j)$ is visited twice by sub-path $u_{k} \rightarrow v_{k} \rightarrow u_{k+1}$ (back and forth). Since $Z_{i j} / \sigma_{i j}$ has i.i.d. standard normal distribution, we have

$$
\begin{aligned}
& \mathbb{E} \operatorname{tr}\left\{\left(Z Z^{\top}-\mathbb{E} Z Z^{\top}\right)^{q}\right\}=\sum_{\mathbf{c} \in\left(\left[p_{1}\right] \times\left[p_{2}\right]\right)^{q}} \prod_{(i, j) \in\left[p_{1}\right] \times\left[p_{2}\right]} \mathbb{E} Z_{i j}^{\alpha_{i j}(\mathbf{c})}\left(Z_{i j}^{2}-\sigma_{i j}^{2}\right)^{\beta_{i j}(\mathbf{c})} \\
= & \sum_{\mathbf{c} \in\left(\left[p_{1}\right] \times\left[p_{2}\right]\right)^{q}} \prod_{(i, j) \in\left[p_{1}\right] \times\left[p_{2}\right]} \sigma_{i j}^{\alpha_{i j}(\mathbf{c})+2 \beta_{i j}(\mathbf{c})} \prod_{(i, j) \in\left[p_{1}\right] \times\left[p_{2}\right]} \mathbb{E} G^{\alpha_{i j}(\mathbf{c})}\left(G^{2}-1\right)^{\beta_{i j}(\mathbf{c})} \\
= & \sum_{\mathbf{c} \in\left(\left[p_{1}\right] \times\left[p_{2}\right]\right)^{q}} \prod_{k=1}^{q} \sigma_{u_{k}, v_{k}} \sigma_{u_{k+1}, v_{k}} \prod_{(i, j) \in\left[p_{1}\right] \times\left[p_{2}\right]} \mathbb{E} G^{\alpha_{i j}(\mathbf{c})}\left(G^{2}-1\right)^{\beta_{i j}(\mathbf{c})} .
\end{aligned}
$$

Here $G$ denotes a $N(0,1)$ random variable. Next, let $m_{\alpha, \beta}(\mathbf{c})$ be the number of edges which appear $\alpha$ times in $\left(u_{k} \rightarrow v_{k}\right)$ or $\left(v_{k} \rightarrow u_{k+1}\right)$ with $u_{k} \neq u_{k+1}$, and $\beta$ times in $\left(u_{k} \rightarrow v_{k} \rightarrow u_{k+1}\right)$ with $u_{k}=u_{k+1}$. More rigorously,

$$
\begin{array}{r}
m_{\alpha, \beta}(\mathbf{c}):=\operatorname{Card}\left\{(i, j) \in\left[p_{1}\right] \times\left[p_{2}\right]: \beta=\left|\left\{k: u_{k}=u_{k+1}=i, v_{k}=j\right\}\right|,\right. \\
\alpha=\mid\left\{k: \text { exactly one of } u_{k} \text { or } u_{k+1}=i, v_{k}=j \mid\right\} .
\end{array}
$$

For any cycle $\mathbf{c}$, we define its shape $\mathbf{s}(\mathbf{u})$ by relabeling the vertices in order of appearance. For example, the cycle $2 \rightarrow 4^{\prime} \rightarrow 3 \rightarrow 2^{\prime} \rightarrow 2 \rightarrow 4^{\prime} \rightarrow 5 \rightarrow 1^{\prime} \rightarrow 2$ has shape $1 \rightarrow 1^{\prime} \rightarrow$ 
$2 \rightarrow 2^{\prime} \rightarrow 1 \rightarrow 1^{\prime} \rightarrow 3 \rightarrow 3^{\prime} \rightarrow 1$. Here $i$ denotes the left vertex while $i^{\prime}$ denotes the right vertex. It is easy to see for any two cycles $\mathbf{c}$ and $\mathbf{c}^{\prime}$ with the same shape, we must have $m_{\alpha, \beta}(\mathbf{c})=m_{\alpha, \beta}\left(\mathbf{c}^{\prime}\right)$. Thus we can well define $m_{\alpha, \beta}(\mathbf{s}(\mathbf{c})):=m_{\alpha, \beta}(\mathbf{c})$. Based on previous discussions,

$$
\prod_{(i, j) \in\left[p_{1}\right] \times\left[p_{2}\right]} \mathbb{E} G^{\alpha_{i j}(\mathbf{c})}\left(G^{2}-1\right)^{\beta_{i j}(\mathbf{c})}=\prod_{\alpha, \beta \geq 0}\left\{\mathbb{E} G^{\alpha}\left(G^{2}-1\right)^{\beta}\right\}^{m_{\alpha, \beta}(\mathbf{s}(\mathbf{c}))} .
$$

Then a natural observation is that $\mathbb{E} G^{\alpha}\left(G^{2}-1\right)^{\beta} \geq 0$ for all non-negative $\alpha, \beta$ and $\mathbb{E} G^{\alpha}\left(G^{2}-1\right)^{\beta}=0$ if and only if $\alpha$ is an odd or $\alpha=0, \beta=1$ (see Lemma 5.2 in Appendix A for details). We then define even shape set $\mathcal{S}_{p_{1}, p_{2}}$ as

$$
\mathcal{S}_{p_{1}, p_{2}}=\left\{\mathbf{s}(\mathbf{c}): m_{\alpha, \beta}(\mathbf{s}(\mathbf{c}))=0 \text { for all } \alpha, \beta \text { s.t. } \alpha \text { is an odd or } \alpha=0, \beta=1\right\} \text {. }
$$

Then the right hand side of (5.5) is nonzero only for $\mathbf{s}(\mathbf{c}) \in \mathcal{S}_{p_{1}, p_{2}}$ and the expansion (5.3) can be further rewritten as

$$
\begin{aligned}
& \mathbb{E} \operatorname{tr}\left\{\left(Z Z^{\top}-\mathbb{E} Z Z^{\top}\right)^{q}\right\} \\
= & \sum_{\mathbf{s}_{0} \in \mathcal{S}_{p_{1}, p_{2}}} \sum_{\mathbf{c}: \mathbf{s}(\mathbf{c})=\mathbf{s}_{0}} \prod_{k=1}^{q} \sigma_{u_{k}, v_{k}} \sigma_{u_{k+1}, v_{k}} \prod_{\alpha, \beta \geq 0}\left\{\mathbb{E} G^{\alpha}\left(G^{2}-1\right)^{\beta}\right\}^{m_{\alpha, \beta}\left(\mathbf{s}_{\mathbf{o}}\right)} \\
= & \sum_{\mathbf{s}_{0} \in \mathcal{S}_{p_{1}, p_{2}}} \prod_{\alpha, \beta \geq 0}\left\{\mathbb{E} G^{\alpha}\left(G^{2}-1\right)^{\beta}\right\}^{m_{\alpha, \beta}\left(\mathbf{s}_{0}\right)} \cdot \sum_{\mathbf{c}: \mathbf{s}(\mathbf{c})=\mathbf{s}_{0}} \prod_{k=1}^{q} \sigma_{u_{k}, v_{k}} \sigma_{u_{k+1}, v_{k}} .
\end{aligned}
$$

Now denote $m_{L}\left(\mathbf{s}_{0}\right)$ and $m_{R}\left(\mathbf{s}_{0}\right)$ be the number of distinct left and right nodes that is visited by cycles with shape $\mathbf{s}_{0}$, we have the following lemma:

Lemma 5.1. Suppose $\sigma_{*} \leq 1$. Then for any shape $\mathbf{s}_{0} \in \mathcal{S}_{p_{1}, p_{2}}$,

$$
\sum_{\mathbf{c}: \mathbf{s}(\mathbf{c})=\mathbf{s}_{0}} \prod_{k=1}^{q} \sigma_{u_{k}, v_{k}} \sigma_{u_{k+1}, v_{k}} \leq\left(p_{1} \sigma_{C}^{2 m_{L}\left(\mathbf{s}_{0}\right)-2} \sigma_{R}^{2 m_{R}\left(\mathbf{s}_{0}\right)}\right) \wedge\left(p_{2} \sigma_{C}^{2 m_{L}\left(\mathbf{s}_{0}\right)} \sigma_{R}^{2 m_{R}\left(\mathbf{s}_{0}\right)-2}\right) .
$$

Proof. The proof of Lemma 5.1 is an analogue of [4, Lemma 2.5]. We first show

$$
\sum_{\mathbf{c}: \mathbf{s}(\mathbf{c})=\mathbf{s}_{0}} \prod_{k=1}^{q} \sigma_{u_{k}, v_{k}} \sigma_{u_{k+1}, v_{k}} \leq p_{1} \sigma_{C}^{2 m_{L}\left(\mathbf{s}_{0}\right)-2} \sigma_{R}^{2 m_{R}\left(\mathbf{s}_{0}\right)}
$$

Suppose $\mathbf{s}_{0}=\left(s_{1}, s_{1}^{\prime}, \ldots, s_{q}, s_{q}^{\prime}\right)$, let $l(k)=\min \left\{j: s_{j}=k\right\}$, i.e., the first time in any cycle of shape $\mathbf{s}_{0}$ at which its $k$ th distinct left vertex is visited. Similarly we define $r(k)=\min \left\{j: s_{j}^{\prime}=k\right\}$. Now let $\mathbf{c}=\left(u_{1}, v_{1}, \cdots u_{q}, v_{q}\right)$ be a cycle with shape $\mathbf{s}_{0}$. Then the following $m_{L}\left(\mathbf{s}_{0}\right)$ distinct edges from right vertex to left vertex will appear in order: $v_{l(2)-1} \rightarrow u_{l(2)}, v_{l(3)-1} \rightarrow u_{l(3)}, \cdots, v_{l\left(m_{L}\left(\mathbf{s}_{0}\right)\right)-1} \rightarrow u_{l\left(m_{L}\left(\mathbf{s}_{0}\right)\right)}$. Similarly, we have $m_{R}\left(\mathbf{s}_{0}\right)$ edges from left vertex to right vertex: $u_{r(1)} \rightarrow v_{r(1)}, u_{r(2)} \rightarrow v_{r(2)}, \cdots, u_{r\left(m_{R}\left(\mathbf{s}_{0}\right)\right)} \rightarrow$ $v_{r\left(m_{R}\left(\mathbf{s}_{0}\right)\right)}$. In addition, these $m_{L}+m_{R}-1$ edges are distinct by the definition of $l(k)$ and $r(k)$. We claim each of these $m_{L}+m_{R}-1$ edges appear at least twice. Suppose one of the above edges only appear once, then we must have $m_{1,0}(\mathbf{s}(\mathbf{c})) \geq 1$, which contradicts 
$\mathbf{s}_{0} \in \mathcal{S}_{p_{1}+p_{2}}$. Now for a fixed starting vertex $u_{1}=u \in\left[p_{1}\right]$, we can bound

$$
\begin{aligned}
& \sum_{\substack{\mathbf{c}: u_{1}=u \\
\mathbf{s}(\mathbf{c})=\mathbf{s}_{0}}} \prod_{k=1}^{q} \sigma_{u_{k}, v_{k}} \sigma_{u_{k+1}, v_{k}} \\
& \leq \sum_{\substack{\mathbf{c}: u_{1}=u \\
\mathbf{s}(\mathbf{c})=\mathbf{s}_{0}}}\left(\sigma_{u_{r(1)}, v_{r(1)}}^{2} \cdots \sigma_{u_{r\left(m_{R}\left(\mathbf{s}_{0}\right)\right)}, v_{r\left(m_{R}\left(\mathbf{s}_{0}\right)\right)}}^{2}\right) \cdot\left(\sigma_{u_{l(2)}, v_{l(2)-1}}^{2} \cdots \sigma_{u_{l\left(m_{L}\left(\mathbf{s}_{0}\right)\right)}, u_{l\left(m_{R}\left(\mathbf{s}_{0}\right)\right)-1}}^{2}\right) \\
& =\sum_{\substack{a_{2} \neq \cdots \neq a_{m_{L}\left(\mathrm{~s}_{0}\right)} \in\left[p_{1}\right] \\
b_{1} \neq \cdots \neq b_{m_{R}\left(\mathrm{~s}_{0}\right)} \in\left[p_{2}\right]}}\left(\sigma_{a_{s_{r(1)}}^{2}, b_{1}}^{2} \cdots \sigma_{a_{s_{r\left(m_{R}\left(\mathrm{~s}_{0}\right)\right)}}^{2}, b_{m_{R}\left(\mathrm{~s}_{0}\right)}}^{2}\right) \\
& \cdot\left(\sigma_{a_{2}, b_{s_{l(2)-1}^{\prime}}^{2}}^{2} \cdots \sigma_{a_{m_{L}\left(\mathrm{~s}_{0}\right)}, b_{s_{l\left(m_{R}\left(\mathrm{~s}_{0}\right)\right)-1}^{\prime}}^{2}}^{2}\right) \\
& \leq \sigma_{R}^{2 m_{R}\left(\mathbf{s}_{0}\right)} \sigma_{C}^{2\left(m_{L}\left(\mathbf{s}_{0}\right)-1\right)} .
\end{aligned}
$$

Then (5.8) follows by taking different initial vertices $u \in\left[p_{1}\right]$. Similarly we can show

$$
\sum_{\mathbf{c}: \mathbf{s}(\mathbf{c})=\mathbf{s}_{0}} \prod_{k=1}^{q} \sigma_{u_{k}, v_{k}} \sigma_{u_{k+1}, v_{k}} \leq p_{2} \sigma_{C}^{2 m_{L}\left(\mathbf{s}_{0}\right)} \sigma_{R}^{2 m_{R}\left(\mathbf{s}_{0}\right)-2}
$$

and the proof is complete.

Combining (5.7) and Lemma 5.1, we obtain

$$
\begin{aligned}
& \mathbb{E} \operatorname{tr}\left\{\left(Z Z^{\top}-\mathbb{E} Z Z^{\top}\right)^{q}\right\} \\
\leq & \sum_{\mathbf{s}_{0} \in \mathcal{S}_{p_{1}, p_{2}}} \prod_{\alpha, \beta \geq 0}\left\{\mathbb{E} G^{\alpha}\left(G^{2}-1\right)^{\beta}\right\}^{m_{\alpha, \beta}\left(\mathbf{s}_{0}\right)} \\
& \cdot\left\{p_{1} \sigma_{C}^{2\left(m_{L}\left(\mathbf{s}_{0}\right)-1\right)} \sigma_{R}^{2 m_{R}\left(\mathbf{s}_{0}\right)}\right\} \wedge\left\{p_{2} \sigma_{C}^{2 m_{L}\left(\mathbf{s}_{0}\right)} \sigma_{R}^{2\left(m_{R}\left(\mathbf{s}_{0}\right)-1\right)}\right\} .
\end{aligned}
$$

Step 2. Next, we consider the expansion for $\operatorname{Etr}\left(\left(H H^{\top}\right)^{q}\right)$, where $H \in \mathbb{R}^{m_{1} \times m_{2}}$ is with i.i.d. standard Gaussian entries. We similarly expand as Step 1 to obtain

$$
\begin{aligned}
& \mathbb{E} \operatorname{tr}\left(\left(H H^{\top}-m_{2} I_{m_{1}}\right)^{q}\right) \\
= & \sum_{\mathbf{s}_{0} \in \mathcal{S}_{p_{1}, p_{2}}} \prod_{\alpha, \beta \geq 0}\left\{\mathbb{E} G^{\alpha}\left(G^{2}-1\right)^{\beta}\right\}^{m_{\alpha, \beta}\left(\mathbf{s}_{0}\right)} \cdot\left|\left\{\mathbf{c}: \mathbf{s}(\mathbf{c})=\mathbf{s}_{0}\right\}\right| \\
= & \sum_{\mathbf{s}_{0} \in \mathcal{S}_{p_{1}, p_{2}}} \prod_{\alpha, \beta \geq 0} \mathbb{E}\left\{G^{\alpha}\left(G^{2}-1\right)^{\beta}\right\}^{m_{\alpha, \beta}\left(\mathbf{s}_{0}\right)} \\
& \cdot m_{1}\left(m_{1}-1\right) \cdots\left(m_{1}-m_{L}\left(\mathbf{s}_{0}\right)+1\right) m_{2}\left(m_{2}-1\right) \cdots\left(m_{2}-m_{R}\left(\mathbf{s}_{0}\right)+1\right)
\end{aligned}
$$

Provided that $m_{1}=\left\lceil\sigma_{C}^{2}\right\rceil+q-1$ and $m_{2}=\left\lceil\sigma_{R}^{2}\right\rceil+q-1, m_{L}\left(\mathbf{s}_{0}\right), m_{R}\left(\mathbf{s}_{0}\right) \leq q$, we have

$$
\begin{aligned}
& m_{1}\left(m_{1}-1\right) \cdots\left(m_{1}-m_{L}\left(\mathbf{s}_{0}\right)+1\right) \cdot m_{2}\left(m_{2}-1\right) \cdots\left(m_{2}-m_{R}\left(\mathbf{s}_{0}\right)+1\right) \\
\geq & m_{1} \cdot\left(m_{1}-m_{L}\left(\mathbf{s}_{0}\right)+1\right)^{m_{L}\left(\mathbf{s}_{0}\right)-1} \cdot\left(m_{1}-m_{R}\left(\mathbf{s}_{0}\right)+1\right)^{m_{R}\left(\mathbf{s}_{0}\right)} \\
\geq & m_{1} \sigma_{C}^{2 m_{L}\left(\mathbf{s}_{0}\right)-2} \cdot \sigma_{R}^{2 m_{R}\left(\mathbf{s}_{0}\right)} .
\end{aligned}
$$

Similarly,

$$
\begin{aligned}
& m_{1}\left(m_{1}-1\right) \cdots\left(m_{1}-m_{L}\left(\mathbf{s}_{0}\right)+1\right) \cdot m_{2}\left(m_{2}-1\right) \cdots\left(m_{2}-m_{R}\left(\mathbf{s}_{0}\right)+1\right) \\
\geq & \sigma_{C}^{2 m_{L}\left(\mathbf{s}_{0}\right)} \cdot m_{2} \sigma_{R}^{2 m_{R}\left(\mathbf{s}_{0}\right)-2} .
\end{aligned}
$$


These all together imply

$$
\begin{aligned}
\mathbb{E} \operatorname{tr}\left(\left(H H^{\top}-m_{2} I_{m_{1}}\right)^{q}\right) \geq & \sum_{\mathbf{s}_{0} \in \mathcal{S}_{p_{1}+p_{2}}} \prod_{\alpha, \beta \geq 0} \mathbb{E}\left\{G^{\alpha}\left(G^{2}-1\right)^{\beta}\right\}^{m_{\alpha, \beta}\left(\mathbf{s}_{0}\right)} \\
& \cdot\left\{m_{1} \sigma_{C}^{2 m_{L}\left(\mathbf{s}_{0}\right)-2} \cdot \sigma_{R}^{2 m_{R}\left(\mathbf{s}_{0}\right)}\right\} \vee\left\{m_{2} \sigma_{C}^{2 m_{L}\left(\mathbf{s}_{0}\right)} \cdot \sigma_{R}^{2 m_{R}\left(\mathbf{s}_{0}\right)-2}\right\} .
\end{aligned}
$$

By comparing (5.9) and (5.10), we have finally proved that

$$
\operatorname{Etr}\left\{\left(Z Z^{\top}-\mathbb{E} Z Z^{\top}\right)^{q}\right\} \leq\left(\frac{p_{1}}{m_{1}} \wedge \frac{p_{2}}{m_{2}}\right) \operatorname{Etr}\left\{\left(H H^{\top}-\mathbb{E} H H^{\top}\right)^{q}\right\} .
$$

Proof of Lemma 2.6. Let $W=\max \left\{\sigma_{\max }(H)-\sqrt{m_{2}}-\sqrt{m_{1}}, \sqrt{m_{2}}-\sqrt{m_{1}}-\sigma_{\min }(M), 0\right\}$, by the tail bound of i.i.d. Gaussian matrix (c.f., [31, Corollary 5.35]), $\mathbb{P}(W \geq t) \leq$ $2 \exp \left(-t^{2} / 2\right)$ for all $t \geq 0$. Thus for any $q \geq 1$,

$$
\mathbb{E} W^{q}=q \int_{0}^{\infty} t^{q-1} \mathbb{P}(W \geq t) d t \leq 2 q \int_{0}^{\infty} t^{q-1} \exp \left(-t^{2} / 2\right) d t=2^{\frac{q}{2}} q \Gamma(q / 2) .
$$

Since

$$
\begin{aligned}
\left\|H H^{\top}-\mathbb{E} H H^{\top}\right\| & =\left\|H H^{\top}-m_{2} I_{m_{1}}\right\|=\max \left\{\sigma_{\max }^{2}(H)-m_{2}, m_{2}-\sigma_{\min }^{2}(H)\right\} \\
& \leq\left(W+\sqrt{m_{1}}+\sqrt{m_{2}}\right)^{2}-m_{2} \\
& =2 \sqrt{m_{1} m_{2}}+m_{1}+W^{2}+2\left(\sqrt{m_{1}}+\sqrt{m_{2}}\right) W,
\end{aligned}
$$

we have

$$
\begin{aligned}
& \left(\mathbb{E}\left\|H H^{\top}-\mathbb{E} H H^{\top}\right\|^{q}\right)^{1 / q} \\
\leq & 2 \sqrt{m_{1} m_{2}}+m_{1}+\left(\mathbb{E} W^{2 q}\right)^{1 / q}+2\left(\sqrt{m_{1}}+\sqrt{m_{2}}\right)\left(\mathbb{E} W^{q}\right)^{1 / q} \\
\leq & 2 \sqrt{m_{1} m_{2}}+m_{1}+\left(2^{q+1} q \Gamma(q)\right)^{1 / q}+2\left(\sqrt{m_{1}}+\sqrt{m_{2}}\right)\left(2^{\frac{q}{2}} q \Gamma(q / 2)\right)^{1 / q} .
\end{aligned}
$$

Next we claim

$$
\left(2^{q+1} q \Gamma(q)\right)^{1 / q} \leq 2 q, \quad\left(2^{\frac{q}{2}} q \Gamma(q / 2)\right)^{1 / q} \leq 2 q^{1 / 2} .
$$

One can verify (5.12) for $2 \leq q \leq 10$ by calculation. When $q \geq 11$, (5.12) can be verified by the Gamma function upper bound in [6]. In summary, we have

$$
\left(\mathbb{E}\left\|H H^{\top}-\mathbb{E} H H^{\top}\right\|^{q}\right)^{1 / q} \leq 2 \sqrt{m_{1} m_{2}}+m_{1}+4\left(\sqrt{m_{1}}+\sqrt{m_{2}}\right) \sqrt{q}+2 q .
$$

which has finished the proof of the first part of this lemma.

For the second part, when $m_{1} \leq m_{2}$, since $H H^{\top}-\mathbb{E} H H^{\top}$ is an $m_{1}$-by- $m_{1}$ matrix, we know $\operatorname{tr}\left(\left(H H^{\top}-\mathbb{E} H H^{\top}\right)^{q}\right)$ is the sum of $m_{1}$ eigenvalues of $\left(H H^{\top}-\mathbb{E} H H^{\top}\right)^{q}$, while each of these eigenvalues are no more than $\left\|H H^{\top}-\mathbb{E} H H^{\top}\right\|^{q}$. Thus,

$$
\begin{gathered}
\operatorname{Etr}\left\{\left(H H^{\top}-\mathbb{E} H H^{\top}\right)^{q}\right\} \leq \mathbb{E} m_{1}\left\|H H^{\top}-\mathbb{E} H H^{\top}\right\|^{q} \\
\leq\left(m_{1} \wedge m_{2}\right) \cdot\left(2 \sqrt{m_{1} m_{2}}+m_{1}+4\left(\sqrt{m_{1}}+\sqrt{m_{2}}\right) \sqrt{q}+2 q\right)^{q} .
\end{gathered}
$$

When $m_{1}>m_{2}$, we shall note that $\operatorname{rank}\left(H H^{\top}\right) \leq m_{2}$ and $\mathbb{E} H H^{\top}=m_{2} I_{m_{1}}$. Then,

$$
\left(H H^{\top}-\mathbb{E} H H^{\top}\right)^{q}-(-1)^{q} m_{2}^{q} I_{m_{1}}=\sum_{k=1}^{q}\left(-m_{2}\right)^{q-k}\left(\begin{array}{c}
q \\
m_{1}
\end{array}\right)\left(H H^{\top}\right)^{k},
$$


which shares the eigenspace of $H H^{\top}$ and has rank no more than $m_{2}$. Thus,

$$
\begin{aligned}
& \mathbb{E} \operatorname{tr}\left\{\left(H H^{\top}-\mathbb{E} H H^{\top}\right)^{q}\right\}=\mathbb{E} \operatorname{tr}\left\{\left(H H^{\top}-\mathbb{E} H H^{\top}\right)^{q}-(-1)^{q} m_{2}^{q} I_{m_{1}}\right\}+\operatorname{tr}\left((-1)^{q} m_{2}^{q} I_{m_{1}}\right) \\
\leq & m_{2} \mathbb{E}\left\|\left(H H^{\top}-\mathbb{E} H H^{\top}\right)^{q}-(-1)^{q} m_{2}^{q} I_{m_{1}}\right\|+m_{1} m_{2}^{q} \\
\leq & m_{2}\left\{\left(2 \sqrt{m_{1} m_{2}}+m_{1}+4\left(\sqrt{m_{1}}+\sqrt{m_{2}}\right) \sqrt{q}+2 q\right)^{q}+m_{2}^{q}\right\}+m_{1} m_{2}^{q} \\
\leq & 2 m_{2}\left(2 \sqrt{m_{1} m_{2}}+m_{1}+4\left(\sqrt{m_{1}}+\sqrt{m_{2}}\right) \sqrt{q}+2 q\right)^{q} \\
= & 2\left(m_{1} \wedge m_{2}\right)\left(2 \sqrt{m_{1} m_{2}}+m_{1}+4\left(\sqrt{m_{1}}+\sqrt{m_{2}}\right) \sqrt{q}+2 q\right)^{q} .
\end{aligned}
$$

where the last inequality is due to $m_{1}>m_{2}$.

Proof of Proposition 2.3. Since $Z_{i j} \stackrel{i i d}{\sim} N(0,1)$, we have $\mathbb{E} Z Z^{\top}=p_{2} I_{p_{1}}$ and

$$
\mathbb{E}\left\|Z Z^{\top}-\mathbb{E} Z Z^{\top}\right\|=\mathbb{E}\left\|Z Z^{\top}-p_{2} I_{p_{1}}\right\| \geq \mathbb{E}\left(\left\|Z Z^{\top}\right\|-p_{2}\right)=\mathbb{E}\|Z\|^{2}-p_{2} .
$$

Since $\|Z\| /\left(\sqrt{p_{1}}+\sqrt{p_{2}}\right) \rightarrow 1$ as $p_{1}, p_{2}$ tend to infinity [31, Theorem 5.31],

$$
\liminf _{p_{1}, p_{2} \rightarrow \infty} \frac{\mathbb{E}\left\|Z Z^{\top}-\mathbb{E} Z Z^{\top}\right\|}{2 \sigma_{C} \sigma_{R}+\sigma_{C}^{2}} \geq \liminf _{p_{1}, p_{2} \rightarrow \infty} \frac{\mathbb{E}\|Z\|^{2}-p_{2}}{2 \sqrt{p_{1} p_{2}}+p_{1}} \geq 1 .
$$

Proof of Theorem 2.8. It suffices to prove the following separate lower bounds to prove this theorem.

$$
\begin{gathered}
\sup _{Z \in \mathcal{F}_{p}\left(\sigma_{*}, \sigma_{C}, \sigma_{R}\right)} \mathbb{E}\left\|Z Z^{\top}-\mathbb{E} Z Z^{\top}\right\| \gtrsim \sigma_{C}^{2} ; \\
\sup _{Z \in \mathcal{F}_{p}\left(\sigma_{*}, \sigma_{C}, \sigma_{R}\right)} \mathbb{E}\left\|Z Z^{\top}-\mathbb{E} Z Z^{\top}\right\| \gtrsim \sigma_{C} \sigma_{R} ; \\
\sup _{Z \in \mathcal{F}_{p}\left(\sigma_{*}, \sigma_{C}, \sigma_{R}\right)} \mathbb{E}\left\|Z Z^{\top}-\mathbb{E} Z Z^{\top}\right\| \gtrsim \sigma_{R} \sigma_{*} \sqrt{\log p}+\sigma_{*}^{2} \log p .
\end{gathered}
$$

1. We first set $\sigma_{i 1}=\sigma_{C} / \sqrt{p_{1}} ; \sigma_{i j}=0, j \geq 2$. If $Z_{i j} \sim N\left(0, \sigma_{i j}^{2}\right)$ independently, it is easy to check that $Z \in \mathcal{F}_{p_{1}, p_{2}}\left(\sigma_{*}, \sigma_{R}, \sigma_{C}\right)$. Then $Z$ is zero except the first column. Suppose the first column of $Z$ is $z$, then $Z Z^{\top}-\mathbb{E} Z Z^{\top}=z z^{\top}-\frac{\sigma_{C}^{2}}{p_{1}} I_{p_{1}}$,

$$
\begin{aligned}
\mathbb{E}\left\|Z Z^{\top}-\mathbb{E} Z Z^{\top}\right\| & =\mathbb{E}\left\|z z^{\top}-\sigma_{C}^{2} / p_{1}\right\| \geq \mathbb{E}\left\|z z^{\top}\right\|-\sigma_{C}^{2} / p_{1}=\mathbb{E}\|z\|_{2}^{2}-\sigma_{C}^{2} / p_{1} \\
& \geq \sigma_{C}^{2}\left(1-1 / p_{1}\right) \geq c \sigma_{C}^{2},
\end{aligned}
$$

which has shown (5.13).

2. Let $k_{1}=\left\lfloor\sigma_{C}^{2} / \sigma_{*}^{2}\right\rfloor, k_{2}=\left\lfloor\sigma_{R}^{2} / \sigma_{*}^{2}\right\rfloor$. Construct

$$
\sigma_{i j}= \begin{cases}\sigma_{*}, & 1 \leq i \leq k_{1}, 1 \leq j \leq k_{2} \\ 0, & \text { otherwise }\end{cases}
$$

By such a construction, $Z_{i j} \sim N\left(0, \sigma_{*}^{2}\right)$ for $1 \leq i \leq k_{1}, 1 \leq j \leq k_{2} ; Z_{i j}=0$ otherwise. Thus,

$$
\begin{aligned}
& \mathbb{E}\left(Z_{\cdot j} Z_{\cdot j}^{\top}-\mathbb{E} Z_{\cdot j} Z_{\cdot j}^{\top}\right)^{2}=\mathbb{E} Z_{\cdot j} Z_{\cdot j}^{\top} Z_{\cdot j} Z_{\cdot j}^{\top}-\left(\mathbb{E} Z_{\cdot j} Z_{\cdot j}^{\top}\right)^{2} \\
= & \mathbb{E}\left\|Z_{\cdot j}\right\|_{2}^{2} Z \cdot j Z_{\cdot j}^{\top}-\sigma_{*}^{4} I_{k_{1}}=\left(k_{1}+1\right) \sigma_{*}^{4} .
\end{aligned}
$$

Here, the last equality is due to

$$
\left(\mathbb{E}\left\|Z_{\cdot j}\right\|_{2}^{2} Z_{\cdot j} Z_{\cdot j}^{\top}\right)_{i, i^{\prime}}=\mathbb{E}\left\|Z_{\cdot j}\right\|_{2}^{2} Z_{i, j} Z_{i^{\prime}, j}= \begin{cases}\left(k_{1}-1+3\right) \sigma_{*}^{4}, & 1 \leq i=i^{\prime} \leq k_{1} \\ 0, & 1 \leq i \neq i^{\prime} \leq k_{1} .\end{cases}
$$


Thus,

$$
\left\|\sum_{j=1}^{k_{2}} \mathbb{E}\left\{Z_{\cdot j} Z_{\cdot j}^{\top}-\mathbb{E} Z_{\cdot j} Z_{\cdot j}^{\top}\right\}^{2}\right\|=\left\|\left(k_{1}+1\right) k_{2} \sigma_{*}^{4} I\right\|=\left(k_{1}+1\right) k_{2} \sigma_{*}^{4} .
$$

Note that $Z Z^{\top}-\mathbb{E} Z Z^{\top}$ can be decomposed as the sum of independent random matrices,

$$
Z Z^{\top}-\mathbb{E} Z Z^{\top}=\sum_{j=1}^{k_{2}}\left\{Z_{\cdot j} Z_{\cdot j}^{\top}-\mathbb{E} Z_{\cdot j} Z_{\cdot j}^{\top}\right\} .
$$

We apply the bound for expected norm of random matrices sum [29] and obtain

$$
\begin{aligned}
\mathbb{E}\left\|Z Z^{\top}-\mathbb{E} Z Z^{\top}\right\| & \gtrsim \sqrt{\left(k_{1}+1\right) k_{2} \sigma_{*}^{4}}=\sqrt{\left(\left\lfloor\sigma_{C}^{2} / \sigma_{*}^{2}\right\rfloor+1\right) \cdot\left\lfloor\sigma_{R}^{2} / \sigma_{*}^{2}\right\rfloor \cdot \sigma_{*}^{4}} \\
& \geq \sqrt{\left(\sigma_{C}^{2} / \sigma_{*}^{2}\right) \cdot \sigma_{R}^{2} /\left(2 \sigma_{*}^{2}\right) \cdot \sigma_{*}^{4}} \quad\left(\text { since } \sigma_{R} \geq \sigma_{*}\right) \\
& \gtrsim \sigma_{R} \sigma_{C} .
\end{aligned}
$$

We thus have shown (5.14).

3. Set $k_{1}=\left\lfloor\sigma_{C}^{2} / \sigma_{*}^{2}\right\rfloor, k_{2}=\left\lfloor\sigma_{R}^{2} / \sigma_{*}^{2}\right\rfloor, m=\left\lfloor\left(p_{1} / k_{1}\right) \wedge\left(p_{2} / k_{2}\right)\right\rfloor$. If $k_{2} \geq(\log p)^{2}$, then $\sigma_{R} \geq \sigma_{*} \log p$ and (5.15) can be implied by (5.14). So we assume $k_{2} \leq(\log p)^{2}$, thus

$$
k_{1} m \geq k_{1}\left(\frac{p_{1}}{2 k_{1}} \wedge \frac{p_{2}}{2 k_{2}}\right) \geq \frac{p_{1}}{2} \wedge \frac{p_{2}}{2(\log p)^{2}} \geq \frac{1}{2} \frac{p}{(\log p)^{2}}
$$

and $\log \left(k_{1} m\right) \geq c \log p$. Let

$$
\left(\sigma_{i j}\right)=\left[\begin{array}{ccc}
B & & \\
& B & \\
& & \ddots
\end{array}\right]=\operatorname{diag}(\overbrace{B, B, \ldots, B, B}^{m}, 0) \in \mathbb{R}^{p_{1} \times p_{2}}, \quad B=\sigma_{*} 1_{k_{1}} 1_{k_{2}}^{\top} .
$$

Then we can rewrite down $Z$ in rowwise form as

$$
Z=\left[\begin{array}{ccc}
\beta_{1}^{\top} & 0 & 0 \\
\vdots & \vdots & \vdots \\
\beta_{k_{1}}^{\top} & 0 & 0 \\
0 & \beta_{k_{1}+1}^{\top} & 0 \\
\vdots & \vdots & \vdots \\
0 & \beta_{2 k_{1}}^{\top} & 0 \\
0 & 0 & \ddots
\end{array}\right] \in \mathbb{R}^{p_{1} \times p_{2}}, \beta_{1}, \ldots, \beta_{k_{1} m} \in \mathbb{R}^{k_{2}}, \beta_{1}, \ldots, \beta_{k_{1} m} \stackrel{i \text { id }}{\sim} N\left(0, \sigma_{*}^{2} I_{k_{2}}\right) .
$$

By taking a look at the expression of $\left\|Z Z^{\top}-\mathbb{E} Z Z^{\top}\right\|$, we know

$$
\left\|Z Z^{\top}-\mathbb{E} Z Z^{\top}\right\| \geq \max _{1 \leq j \leq k_{1} m}\left|\beta_{j}^{\top} \beta_{j}-k_{2} \sigma_{*}^{2}\right| .
$$

Note that $\beta_{j}^{\top} \beta_{j} / \sigma_{*}^{2} \sim \chi_{k_{2}}^{2}$. By the lower bound of right-tail of Chi-square distribution (Corollary 3 in [37]), we have $\mathbb{P}\left(\beta_{j}^{\top} \beta_{j}-k_{2} \sigma_{*}^{2} \geq \sigma_{*}^{2} x\right) \geq c \exp \left(-C\left(x \wedge \frac{x^{2}}{k_{2}}\right)\right)$. Since

$$
\begin{aligned}
\mathbb{P}\left(\max _{j} \beta_{j}^{\top} \beta_{j}-k_{2} \sigma_{*}^{2}>\sigma_{*}^{2} x\right) & =1-\mathbb{P}\left(\max _{j} \beta_{j}^{\top} \beta_{j}-k_{2} \sigma_{*}^{2} \leq \sigma_{*}^{2} x\right) \\
& =1-\prod_{j=1}^{k_{1} m}\left(1-\mathbb{P}\left(\beta_{j}^{\top} \beta_{j}-k_{2} \sigma_{*}^{2} \geq \sigma_{*}^{2} x\right)\right) \\
& \geq 1-\left(1-c \exp \left(-C\left(x \wedge \frac{x^{2}}{k_{2}}\right)\right)\right)^{k_{1} m}
\end{aligned}
$$


Taking $x=c_{1}\left(\sqrt{k_{2} \log \left(k_{1} m\right)} \vee \log \left(k_{1} m\right)\right)$ for some $c_{1}$ such that $-C\left(x \wedge \frac{x^{2}}{k_{2}}\right) \geq$ $-\log \left(k_{1} m\right)$, we get

$$
\left(1-c \exp \left(-C\left(x \wedge \frac{x^{2}}{k_{2}}\right)\right)\right)^{k_{1} m} \leq\left(1-\frac{c^{\prime}}{\left(k_{1} m\right)}\right)^{k_{1} m} \leq e^{-c^{\prime}} .
$$

Thus,

$$
\begin{aligned}
& \mathbb{E}\left\|Z Z^{\top}-\mathbb{E} Z Z^{\top}\right\| \geq \mathbb{E} \max _{1 \leq j \leq k_{1} m} \beta_{j}^{\top} \beta_{j}-k_{2} \sigma_{*}^{2} \\
\geq & \sup _{x>0} x \sigma_{*}^{2} \cdot \mathbb{P}\left(\max _{j} \beta_{j}^{\top} \beta_{j}-k_{2} \sigma_{*}^{2}>x \sigma_{*}^{2}\right) \\
\geq & c_{1}\left(1-e^{-c^{\prime}}\right)\left(\sqrt{k_{2} \log \left(k_{1} m\right)} \vee \log \left(k_{1} m\right)\right) \\
\gtrsim & c \sigma_{*}^{2}\left(\sqrt{k_{2} \log \left(k_{1} m\right)}+\log \left(k_{1} m\right)\right) \gtrsim c \sigma_{*} \sigma_{R} \sqrt{\log p}+c \sigma_{*}^{2} \log p .
\end{aligned}
$$

\subsection{Proofs for non-Gaussian distributions}

In this section, we collect the proofs of concentration for the non-Gaussian Wisharttype matrix (Lemma 3.1, Theorem 3.4 and Theorem 3.5) in Section 3.1.

Proof of Lemma 3.1. Following the notations and proof idea of Lemma 2.4, we have the same expansion of $\operatorname{Etr}\left\{\left(Z Z^{\top}-\mathbb{E} Z Z^{\top}\right)^{q}\right\}$ as (5.3):

$$
\begin{aligned}
& \operatorname{Etr}\left\{\left(Z Z^{\top}-\mathbb{E} Z Z^{\top}\right)^{q}\right\}=\sum_{\mathbf{c} \in\left(\left[p_{1}\right] \times\left[p_{2}\right]\right)^{q}} \prod_{(i, j) \in\left[p_{1}\right] \times\left[p_{2}\right]} \mathbb{E} Z_{i j}^{\alpha_{i j}(\mathbf{c})}\left(Z_{i j}^{2}-\sigma_{i j}^{2}\right)^{\beta_{i j}(\mathbf{c})} \\
= & \sum_{\mathbf{c} \in\left(\left[p_{1}\right] \times\left[p_{2}\right]\right)^{q}} \prod_{k=1}^{q} \sigma_{u_{k}, v_{k}} \sigma_{u_{k+1}, v_{k}} \prod_{(i, j) \in\left[p_{1}\right] \times\left[p_{2}\right]} \mathbb{E} G_{i j}^{\alpha_{i j}(\mathbf{c})}\left(G_{i j}^{2}-1\right)^{\beta_{i j}(\mathbf{c})},
\end{aligned}
$$

where $G_{i j}:=Z_{i j} / \sigma_{i j}$. Different from (5.3), $E_{i j}$ in (5.16) may not have $N(0,1)$ distribution. To overcome this difficulty, we introduce the following lemma to bound $\mathbb{E} E_{i j}^{\alpha}\left(E_{i j}^{2}-1\right)^{\beta}$ via a Gaussian analogue.

Lemma 5.2 (Gaussian moments). Suppose $G \sim N(0,1), \alpha, \beta$ are non-negative integers, then

$$
\begin{cases}(\alpha+2 \beta-1) ! ! \geq \mathbb{E} G^{\alpha}\left(G^{2}-1\right)^{\beta} \geq(\alpha+2 \beta-3) ! ! \cdot(\alpha+\beta-1), & \text { if } \alpha \text { is even } \\ \mathbb{E} G^{\alpha}\left(G^{2}-1\right)^{\beta}=0, & \text { if } \alpha \text { is odd } .\end{cases}
$$

Here for odd $k, k ! !=k(k-2) \cdots 1$. Especially, $(-1) ! !=1,(-3) ! !=-1$. More generally, if $Z$ has symmetric distribution and satisfies

$$
\operatorname{Var}(Z)=1, \quad\|Z\|_{\psi_{2}}=\sup _{q \geq 1} q^{-1 / 2}\left(\mathbb{E}|Z|^{q}\right)^{1 / q} \leq \kappa
$$

Then for any integers $\alpha, \beta \geq 0$,

$$
\left|\mathbb{E} Z^{\alpha}\left(Z^{2}-1\right)^{\beta}\right| \leq(C \kappa)^{\alpha+2 \beta} \mathbb{E} G^{\alpha}\left(G^{2}-1\right)^{\beta}
$$

for some uniform constant $C>0$.

Proof of Lemma 5.2. See Appendix. 
Now, Combining (5.16) and (5.19), we have $\operatorname{Etr}\left\{\left(Z Z^{\top}-\mathbb{E} Z Z^{\top}\right)^{q}\right\}$

$$
\begin{aligned}
& \leq \sum_{\mathbf{c} \in\left(\left[p_{1}\right] \times\left[p_{2}\right]\right)^{q}} \prod_{k=1}^{q} \sigma_{u_{k}, v_{k}} \sigma_{u_{k+1}, v_{k}} \prod_{(i, j) \in\left[p_{1}\right] \times\left[p_{2}\right]}(C \kappa)^{\alpha_{i j}(\mathbf{c})+2 \beta_{i j}(\mathbf{c})} \mathbb{E} E_{i j}^{\alpha_{i j}(\mathbf{c})}\left(E_{i j}^{2}-1\right)^{\beta_{i j}(\mathbf{c})} \\
& =(C \kappa)^{2 q} \sum_{\mathbf{c} \in\left(\left[p_{1}\right] \times\left[p_{2}\right]\right)^{q}} \prod_{k=1}^{q} \sigma_{u_{k}, v_{k}} \sigma_{u_{k+1}, v_{k}} \prod_{(i, j) \in\left[p_{1}\right] \times\left[p_{2}\right]} \mathbb{E} G^{\alpha_{i j}(\mathbf{c})}\left(G^{2}-1\right)^{\beta_{i j}(\mathbf{c})} .
\end{aligned}
$$

The rest of the proof can similarly proceed as we did in proving Lemma 2.4.

Proof of Theorem 3.4. Let $b:=2 / \alpha \geq 2$ and $E_{i j}:=Z_{i j} / \sigma_{i j}$. By definition, we have $\sup _{q} q^{-\frac{b}{2}}\left(\mathbb{E}\left|E_{i j}\right|^{q}\right)^{1 / q} \leq \kappa$. Thus for any $\alpha, \beta \geq 0$,

$$
\begin{aligned}
\left|\mathbb{E} E_{i j}^{\alpha}\left(E_{i j}^{2}-1\right)^{\beta}\right| & \leq\left|\mathbb{E} E_{i j}^{\alpha}\left(E_{i j}^{2}-1\right) 1_{\left\{\left|E_{i j}\right| \leq 1\right\}}+\mathbb{E} E_{i j}^{\alpha}\left(E_{i j}^{2}-1\right)^{\beta} 1_{\left\{\left|E_{i j}\right|>1\right\}}\right| \\
& \leq 1+\mathbb{E}\left|E_{i j}\right|^{\alpha+2 \beta} \leq(C \kappa)^{\alpha+2 \beta}(\alpha+2 \beta)^{\frac{b(\alpha+2 \beta)}{2}} .
\end{aligned}
$$

We introduce the following technical lemma.

Lemma 5.3. Let $G, \tilde{G}$ be independent $N(0,1)$ and let $F_{i j}$ be i.i.d. copy of $G|\tilde{G}|^{b-1}$. Then,

$$
\mathbb{E} E_{i j}^{\alpha}\left(E_{i j}^{2}-1\right)^{\beta} \leq\left(C_{b} \kappa\right)^{\alpha+2 \beta} \mathbb{E} F_{i j}^{\alpha}\left(F_{i j}^{2}-1\right)^{\beta} .
$$

Here $C_{b}$ is some constant which only depend on $b$.

Proof of Lemma 5.3. See Appendix.

Now let $G_{i j}, \tilde{G}_{i j}$ be i.i.d. $N(0,1)$ and define $F_{i j}=G_{i j}\left|\tilde{G}_{i j}\right|^{b-1}$. Let $\tilde{Z}$ be a random matrix with entries $\tilde{Z}_{i j}=\sigma_{i j} F_{i j}$. Then, by Lemma 5.3 and the similar proof in Lemma 3.1, we have

$$
\mathbb{E} \operatorname{tr}\left\{\left(Z Z^{\top}-\mathbb{E} Z Z^{\top}\right)^{q}\right\} \leq\left(C_{b} \kappa\right)^{2 q} \mathbb{E} \operatorname{tr}\left\{\left(\tilde{Z} \tilde{Z}-\mathbb{E} \tilde{Z} \tilde{Z}^{\top}\right)^{q}\right\}
$$

Thus,

$$
\begin{aligned}
\mathbb{E}\left\|Z Z^{\top}-\mathbb{E} Z Z^{\top}\right\| & \leq\left(\mathbb{E} \operatorname{tr}\left\{\left(Z Z^{\top}-\mathbb{E} Z Z^{\top}\right)^{2 q}\right\}\right)^{1 / 2 q} \\
& \leq\left(C_{b} \kappa\right)^{2}\left(\mathbb{E} \operatorname{tr}\left\{\left(\tilde{Z} \tilde{Z}^{\top}-\mathbb{E} \tilde{Z} \tilde{Z}^{\top}\right)^{2 q}\right\}\right)^{1 / 2 q}
\end{aligned}
$$

Let $q=\left\lceil\log \left(p_{1} \wedge p_{2}\right)\right\rceil$, now it suffices to upper bound $\left(\mathbb{E}\left\|\tilde{Z} \tilde{Z}^{\top}-\mathbb{E} Z \tilde{Z}^{\top}\right\|^{2 q}\right)^{1 / 2 q}$. We define $\tilde{\sigma}_{C}^{2}=\max _{j} \sum_{i=1}^{p_{1}} \sigma_{i j}^{2}\left|\tilde{G}_{i j}\right|^{2 b-2}, \tilde{\sigma}_{R}^{2}=\max _{i} \sum_{j=1}^{p_{2}} \sigma_{i j}^{2}\left|\tilde{G}_{i j}\right|^{2 b-2}$ and $\tilde{\sigma}_{*}=\max _{i} \sigma_{i j}\left|\tilde{G}_{i j}\right|^{b-1}$ and apply Theorem 2.1 conditionally on $\tilde{G}$ :

$$
\begin{aligned}
& \mathbb{E}\left[\operatorname{tr}\left\{\left(\tilde{Z} \tilde{Z}^{\top}-\mathbb{E} \tilde{Z}^{\top} \tilde{Z}^{\top}\right)^{2 q}\right\} \mid \tilde{G}\right] \\
& \quad \leq C^{2 q}\left(\tilde{\sigma}_{C}^{2}+\tilde{\sigma}_{C} \tilde{\sigma}_{R}+\sigma_{C} \sigma_{*} \sqrt{\log \left(p_{1} \wedge p_{2}\right)}+\sigma_{*}^{2} \log \left(p_{1} \wedge p_{2}\right)\right)^{2 q} .
\end{aligned}
$$

Then,

$$
\begin{aligned}
& \left(\mathbb{E} \operatorname{tr}\left\{\left(\tilde{Z} \tilde{Z}^{\top}-\mathbb{E} \tilde{Z} \tilde{Z}^{\top}\right)^{2 q}\right\}\right)^{1 / 2 q} \\
& \quad \leq C\left(\left\|\tilde{\sigma}_{C}^{2}\right\|_{2 q}+\left\|\tilde{\sigma}_{C} \sigma_{R}\right\|_{2 q}+\left\|\tilde{\sigma}_{R} \tilde{\sigma}_{*}\right\|_{2 q} \sqrt{\log \left(p_{1} \wedge p_{2}\right)}+\left\|\tilde{\sigma}_{*}^{2}\right\|_{2 q} \log \left(p_{1} \wedge p_{2}\right)\right) .
\end{aligned}
$$

Here $\|X\|_{2 q}:=\left(\mathbb{E}|X|^{2 q}\right)^{1 / 2 q}$ is the $\ell_{2 q}$-norm of random variable $X$. Now we bound $\left\|\tilde{\sigma}_{*}^{2}\right\|_{2 q^{\prime}}$ $\left\|\tilde{\sigma}_{R}^{2}\right\|_{2 q}$ and $\left\|\tilde{\sigma}_{C}^{2}\right\|_{2 q}$ separately. 
- $\left\|\tilde{\sigma}_{*}^{2}\right\|_{2 q}$. For any $a>0$, since

$$
\mathbb{P}\left(\max _{i, j}\left|\tilde{G}_{i j}\right|>t\right) \leq 2 \exp \left(-\frac{t^{2}}{2}+\log \left(p_{1} p_{2}\right)\right) \leq 2 \exp \left(-\frac{t^{2}}{4}\right), \quad \forall t>2 \sqrt{\log \left(p_{1} p_{2}\right)},
$$

integration yields

$$
\begin{aligned}
\mathbb{E} \max _{i, j}\left|\tilde{G}_{i j}\right|^{a} & =\int_{0}^{\infty} \mathbb{P}\left(\max _{i, j}\left|\tilde{G}_{i j}\right|>t^{1 / a}\right) d t \leq\left(2 \sqrt{\log \left(p_{1} p_{2}\right)}\right)^{a}+\int_{0}^{\infty} 2 e^{-\frac{t^{2 / a}}{4}} d t \\
& =\left(4 \log \left(p_{1} p_{2}\right)\right)^{a / 2}+4 a \Gamma\left(\frac{a}{2}\right) .
\end{aligned}
$$

Then it follows that

$$
\begin{aligned}
\left\|\tilde{\sigma}_{*}^{2}\right\|_{2 q} & \leq \sigma_{*}^{2}\left(\mathbb{E} \max _{i, j}\left|\tilde{G}_{i j}\right|^{4(b-1) q}\right)^{1 / 2 q} \\
& \leq \sigma_{*}^{2}\left(\left(4 \log \left(p_{1} p_{2}\right)\right)^{2(b-1) q}+16(b-1) q \Gamma(2(b-1) q)\right)^{1 / 2 q} \\
& \lesssim \sigma_{*}^{2}\left(\log \left(p_{1} p_{2}\right)^{b-1}+q^{b-1}\right) \lesssim \sigma_{*}^{2} \log ^{b-1}\left(p_{1} \vee p_{2}\right) .
\end{aligned}
$$

- $\left\|\tilde{\sigma}_{C}^{2}\right\|_{2 q}$ and $\left\|\tilde{\sigma}_{R}^{2}\right\|$. By the moment bound of supremum of empirical process [9, Theorem 11],

$$
\begin{aligned}
\left\|\tilde{\sigma}_{C}^{2}\right\|_{2 q} & =\left\|\max _{j} \sum_{i=1}^{p_{1}} \sigma_{i j}^{2}\left|\tilde{G}_{i j}\right|^{2 b-2}\right\|_{2 q} \\
& \lesssim \mathbb{E} \max _{1 \leq j \leq p_{2}} \sum_{i=1}^{p_{1}} \sigma_{i j}^{2}\left|\tilde{G}_{i j}\right|^{2 b-2}+q\left\|\tilde{\sigma}_{*}^{2}\right\|_{2 q} \\
& \leq \mathbb{E} \max _{j} \sum_{i=1}^{p_{1}}\left(\sigma_{i j}^{2}\left|\tilde{G}_{i j}\right|^{2 b-2}-\mathbb{E} \sigma_{i j}^{2}\left|\tilde{G}_{i j}\right|^{2 b-2}\right)+\sigma_{C}^{2}+q\left\|\tilde{\sigma}_{*}^{2}\right\|_{2 q} .
\end{aligned}
$$

Denote $Y_{j}=\sum_{i=1}^{p_{1}}\left(\sigma_{i j}^{2}\left|\tilde{G}_{i j}\right|^{2 b-2}-\mathbb{E} \sigma_{i j}^{2}\left|\tilde{G}_{i j}\right|^{2 b-2}\right)$, it suffices to bound $\mathbb{E} \max _{j} Y_{j}$. To this end, we introduce the following Generalized Bernstein-Orlicz norm defined in [19]. For a random variable $X$, let

$$
\|X\|_{\Psi_{\alpha, L}}:=\inf \left\{\eta>0: \mathbb{E}\left[\Psi_{\alpha, L}(|X| / \eta)\right] \leq 1\right\}
$$

be the $\Psi_{\alpha, L}$-norm where $\Psi_{\alpha, L}$ is defined via its inverse function

$$
\Psi_{\alpha, L}^{-1}(t):=\sqrt{\log (1+t)}+L(\log (1+t))^{1 / \alpha}, \quad \forall t \geq 0 .
$$

Now fix $j \in\left[p_{2}\right]$ and let $\alpha=1 /(b-1)$ and $L=\frac{4^{b-1} \sigma_{*}^{2}}{\sqrt{2} \sqrt{\sum_{i=1}^{p} \sigma_{i j}^{4}}}$. By [19, Theorem 3.1],

$$
\begin{gathered}
\left\|Y_{j}\right\|_{\Psi_{\alpha, L}} \leq C \sqrt{\sum_{i=1}^{p_{1}} \sigma_{i j}^{4}} \\
\mathbb{P}\left(\left|Y_{j}\right| \geq C \sqrt{\left.\sum_{i=1}^{p_{1}} \sigma_{i j}^{4}\left\{\sqrt{t}+L t^{1 / \alpha}\right\}\right) \leq 2 \exp (-t), \quad t \geq 0 .} .\right.
\end{gathered}
$$

This yields

$$
\mathbb{P}\left(\left|Y_{j}\right| \geq C\left\{\sigma_{C} \sigma_{*} \sqrt{t}+\sigma_{*}^{2} t^{b-1}\right\}\right) \leq 2 \exp (-t), \quad t \geq 0,
$$


which can be rewritten as

$$
\mathbb{P}\left(\left|Y_{j}\right| \geq t\right) \leq 2 \exp \left(-c\left(\frac{t^{2}}{\sigma_{C}^{2} \sigma_{*}^{2}} \wedge\left(\frac{t}{\sigma_{*}^{2}}\right)^{1 /(b-1)}\right)\right), \quad t \geq 0 .
$$

Applying union bound, we get

$$
\mathbb{P}\left(\max _{j}\left|Y_{j}\right| \geq t\right) \leq 2 \exp \left(\log p_{2}-c\left(\frac{t^{2}}{\sigma_{C}^{2} \sigma_{*}^{2}} \wedge\left(\frac{t}{\sigma_{*}^{2}}\right)^{1 /(b-1)}\right)\right), \quad t \geq 0 .
$$

Now it follows that

$$
\begin{aligned}
& \mathbb{E} \max _{j} Y_{j} \leq \mathbb{E} \max _{j}\left|Y_{j}\right|=\int_{0}^{\infty} \mathbb{P}\left(\max _{j}\left|Y_{j}\right|>t\right) d t \\
& \leq C\left(\sigma_{C} \sigma_{*} \sqrt{\log p_{2}}+\sigma_{*}^{2} \log ^{b-1}\left(p_{2}\right)\right) \\
& +\int_{C\left(\sigma_{C} \sigma_{*} \sqrt{\log p_{2}}+\sigma_{*}^{2} \log ^{b-1}\left(p_{2}\right)\right)}^{\infty} \mathbb{P}\left(\max _{j}\left|Y_{j}\right|>t\right) d t \\
& \leq C\left(\sigma_{C} \sigma_{*} \sqrt{\log p_{2}}+\sigma_{*}^{2} \log ^{b-1}\left(p_{2}\right)\right) \\
& +\int_{0}^{\infty}\left(\exp \left(-c \frac{t^{2}}{\sigma_{C} \sigma_{*}}\right)+\exp \left(-c \frac{t^{1 /(b-1)}}{\sigma_{*}^{2 /(b-1)}}\right)\right) d t \\
& \lesssim \sigma_{C} \sigma_{*} \sqrt{\log p_{2}}+\sigma_{*}^{2} \log ^{b-1}\left(p_{2}\right) \\
& \lesssim \sigma_{C}^{2}+\sigma_{*}^{2} \log ^{b-1}\left(p_{2}\right) \text {. }
\end{aligned}
$$

Combining with (5.25), we obtained

$$
\left\|\tilde{\sigma}_{C}^{2}\right\|_{2 q} \lesssim \sigma_{C}^{2}+\sigma_{*}^{2} \log ^{b-1}\left(p_{1} \vee p_{2}\right) \log \left(p_{1} \wedge p_{2}\right)
$$

Similarly we can obtain

$$
\left\|\tilde{\sigma}_{R}\right\|_{2 q} \lesssim \sigma_{R}^{2}+\sigma_{*}^{2} \log ^{b-1}\left(p_{1} \vee p_{2}\right) \log \left(p_{1} \wedge p_{2}\right)
$$

Combining (5.23), (5.24), (5.26), (5.27) and applying Cauchy-Schwarz inequality, we obtain

$$
\begin{gathered}
\mathbb{E}\left\|Z Z^{\top}-\mathbb{E} Z Z^{\top}\right\| \lesssim \sigma_{C}^{2}+\sigma_{R} \sigma_{C}+\sigma_{R} \sigma_{*} \log ^{(b-1) / 2}\left(p_{1} \vee p_{2}\right) \sqrt{\log \left(p_{1} \wedge p_{2}\right)} \\
+\sigma_{*}^{2} \log ^{b-1}\left(p_{1} \vee p_{2}\right) \log \left(p_{1} \wedge p_{2}\right)
\end{gathered}
$$

This completes the proof.

Proof of Theorem 3.5. We first prove the following comparison Lemma.

Lemma 5.4. Suppose $Z$ is a $p_{1}$-by- $p_{2}$ random matrix with independent entries satisfying $\mathbb{E} Z_{i j}=0, \operatorname{Var}\left(Z_{i j}\right)=\sigma_{i j}^{2},|Z| \leq 1$. $H$ is an $m_{1}$-by- $m_{2}$ dimensional matrix with i.i.d. standard Gaussian entries. When $q \geq 1, m_{1}=\left\lceil\sigma_{C}^{2}\right\rceil+q-1, m_{2}=\left\lceil\sigma_{R}^{2}\right\rceil+q-1$, we have

$$
\operatorname{Etr}\left\{\left(Z Z^{\top}-\mathbb{E} Z Z^{\top}\right)^{q}\right\} \leq\left(\frac{p_{1}}{m_{1}} \wedge \frac{p_{2}}{m_{2}}\right) \mathbb{E} \operatorname{tr}\left\{\left(H H^{\top}-\mathbb{E} H H^{\top}\right)^{q}\right\} .
$$

Proof. Recall $Z \in \mathbb{R}^{p_{1} \times p_{2}},|Z| \leq 1$ almost surely, $\mathbb{E} Z_{i j}=0, \operatorname{Var}\left(Z_{i j}\right)=\sigma_{i j}^{2}$. Similarly as the proof of Lemma 2.4, let $\mathbf{c}=\left(u_{1}, v_{1}, \ldots, u_{q}, v_{q}\right) \in\left(\left[p_{1} \times\left[p_{2}\right]\right]\right)^{q}$ be the cycle of length 


\section{Heteroskedastic Wishart-type concentration}

$2 q$ on bipartite graph $\left[p_{1}\right] \rightarrow\left[p_{2}\right], \alpha_{i j}(\mathbf{c})$ and $\beta_{i j}(\mathbf{c})$ be defined as (5.2). We similarly have the following expansion,

$$
\begin{aligned}
& \mathbb{E}\left\{\left(Z Z^{\top}-\mathbb{E} Z Z^{\top}\right)^{q}\right\}=\sum_{u_{1}, \ldots, u_{q} \in\left[p_{1}\right]} \mathbb{E} \prod_{j=1}^{q}\left(Z Z^{\top}-\mathbb{E} Z Z^{\top}\right)_{u_{j}, u_{j+1}} \\
= & \mathbb{E} \sum_{u_{1}, \ldots, u_{q} \in\left[p_{1}\right]} \prod_{j=1}^{q}\left\{\sum_{v_{j} \in\left[p_{2}\right]} Z_{u_{j}, v_{j}}\left(Z^{\top}\right)_{v_{j}, u_{j+1}}-1_{\left\{u_{j}=u_{j+1}\right\}} \sum_{v_{j} \in\left[p_{2}\right]} \mathbb{E} Z_{u_{j}, v_{j}}^{2}\right\} \\
= & \sum_{\substack{u_{1}, \ldots, u_{q} \in\left[p_{1}\right] \\
v_{1}, \ldots, v_{q} \in\left[p_{2}\right]}} \mathbb{E} \prod_{j=1}^{q}\left(Z_{u_{j}, v_{j}} Z_{u_{j+1}, v_{j}}-\sigma_{u_{j}, v_{j}}^{2} 1_{\left\{u_{j}=u_{j+1}\right\}}\right) \\
= & \sum_{\mathbf{c} \in\left(\left[p_{1}\right] \times\left[p_{2}\right]\right)^{q}} \prod_{(i, j) \in\left[p_{1}\right] \times\left[p_{2}\right]} \mathbb{E} Z_{i j}^{\alpha_{i j}(\mathbf{c})}\left(Z_{i j}^{2}-\sigma_{i j}^{2}\right)^{\beta_{i j}(\mathbf{c})} .
\end{aligned}
$$

Since $Z_{i j}$ is symmetric distributed and $\mathbb{E} Z_{i j}^{2}=\sigma_{i j}^{2}$, we have

$$
\mathbb{E} Z_{i j}^{\alpha}\left(Z_{i j}^{2}-\sigma_{i j}^{2}\right)^{\beta}=0, \quad \text { if } \alpha \text { is odd or }\{\alpha=0, \beta=1\} .
$$

For any $(i, j) \in\left[p_{1}\right] \times\left[p_{2}\right]$, we shall note that $0 \leq Z_{i j}^{2} \leq 1$ and $\left|Z_{i j}^{2}-\sigma_{i j}^{2}\right| \leq 1$. If $\alpha \geq 2$ and $\alpha$ is even,

$$
\mathbb{E}\left|Z_{i j}^{\alpha_{i j}(\mathbf{c})}\left(Z_{i j}^{2}-\sigma_{i j}^{2}\right)^{\beta_{i j}(\mathbf{c})}\right|=\mathbb{E}\left|Z_{i j}^{2}\right| \cdot\left|Z_{i j}^{\alpha_{i j}(\mathbf{c})-2}\left(Z_{i j}^{2}-\sigma_{i j}^{2}\right)^{\beta_{i j}(\mathbf{c})}\right| \leq \mathbb{E} Z_{i j}^{2}=\sigma_{i j}^{2} ;
$$

if $\alpha \geq 0, \beta \geq 2$, one has

$$
\begin{aligned}
& \mathbb{E}\left|Z_{i j}^{\alpha_{i j}(\mathbf{c})}\left(Z_{i j}^{2}-\sigma_{i j}^{2}\right)^{\beta_{i j}(\mathbf{c})}\right| \leq \mathbb{E}\left(Z_{i j}^{2}-\sigma_{i j}^{2}\right)^{2} \cdot\left|Z_{i j}^{\alpha_{i j}(\mathbf{c})}\left(Z_{i j}^{2}-\sigma_{i j}^{2}\right)^{\beta_{i j}(\mathbf{c})-2}\right| \\
\leq & \mathbb{E} Z_{i j}^{4}-\sigma_{i j}^{4} \leq \mathbb{E} Z_{i j}^{2} \cdot\left\|Z_{i j}\right\|_{\infty}^{2}-\sigma_{i j}^{4}=\sigma_{i j}^{2}-\sigma_{i j}^{4} \leq \sigma_{i j}^{2} .
\end{aligned}
$$

Therefore, for any $\alpha, \beta \geq 0$, we have

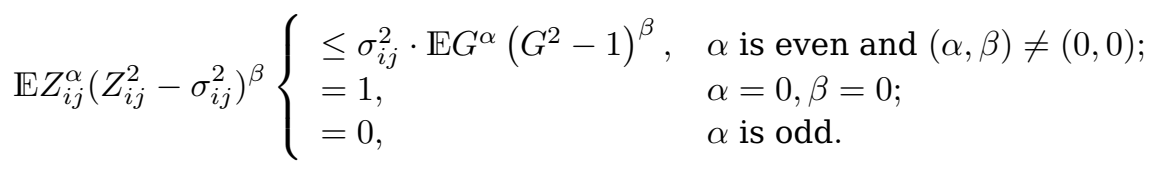

Here, $G \sim N(0,1)$. Thus,

$$
\leq \sum_{\mathbf{c} \in\left(\left[p_{1}\right] \times\left[p_{2}\right]\right)^{q}} \prod_{(i, j) \in\left[p_{1}\right] \times\left[p_{2}\right]} \sigma_{i j}^{2} 1_{\left\{\left(\alpha_{i j}(\mathbf{c}), \beta_{i j}(\mathbf{c})\right) \neq(0,0)\right\}} \mathbb{E} G^{\alpha_{i j}(\mathbf{c})}\left(G^{2}-1\right)^{\beta_{i j}(\mathbf{c})} .
$$

Let $\mathbf{s}$ be the shape of any loop $\mathbf{c} \in\left(\left[p_{1}\right] \times\left[p_{2}\right]\right)^{q}, m_{L}(\mathbf{s})$ and $m_{R}(\mathbf{s})$ be the number of distinct left and right nodes respectively visited by any $\mathbf{c}$ with shape $\mathbf{s} ; m_{\alpha, \beta}(\mathbf{s})=m_{\alpha, \beta}(\mathbf{c})$ is defined as (5.4)). Then,

$$
\begin{aligned}
& \mathbb{E}\left\{\left(Z Z^{\top}-\mathbb{E} Z Z^{\top}\right)^{q}\right\} \\
& \leq \sum_{\mathbf{c} \in\left(\left[p_{1}\right] \times\left[p_{2}\right]\right)^{q}} \prod_{(i, j) \in\left[p_{1}\right] \times\left[p_{2}\right]} \sigma_{i j}^{2} 1_{\left\{\left(\alpha_{i j}(\mathbf{c}), \beta_{i j}(\mathbf{c})\right) \neq(0,0)\right\}} \mathbb{E} G^{\alpha_{i j}(\mathbf{c})}\left(G^{2}-1\right)^{\beta_{i j}(\mathbf{c})} \\
& =\sum_{\mathbf{s}} \sum_{\substack{\mathbf{c}: \\
\mathbf{c} \text { has shape s }}} \prod_{\substack{(i, j) \in\left[p_{1}\right] \times\left[p_{2}\right] \\
\mathbf{c} \text { pass }(i, j) \text { for positive even times }}} \sigma_{i j}^{2} \cdot \prod_{\substack{\alpha, \beta \geq 0 \\
\alpha \text { is even }}}\left\{G^{\alpha}\left(G^{2}-1\right)^{\beta}\right\}^{m_{\alpha, \beta}(\mathbf{s})} \\
& \leq \sum_{\mathbf{s}}\left(p_{1} \sigma_{C}^{2 m_{L}(\mathbf{s})-2} \sigma_{R}^{2 m_{R}(\mathbf{s})} \wedge p_{2} \sigma_{C}^{2 m_{L}(\mathbf{s})} \sigma_{R}^{2 m_{R}(\mathbf{s})-2}\right) \cdot \prod_{\substack{\alpha, \beta \geq 0 \\
\alpha \text { is even }}}\left\{G^{\alpha}\left(G^{2}-1\right)^{\beta}\right\}^{m_{\alpha, \beta}(\mathbf{s})} \text {. }
\end{aligned}
$$


On the other hand, we have

$$
\begin{aligned}
& \mathbb{E} \operatorname{tr}\left(\left(H H^{\top}-\mathbb{E} H H^{\top}\right)^{q}\right)=\mathbb{E} \operatorname{tr}\left(\left(H H^{\top}-m_{2} I_{m_{1}}\right)^{q}\right) \\
= & \sum_{\mathbf{s}} m_{1} \cdots\left(m_{1}-m_{L}(\mathbf{s})+1\right) \cdot m_{2} \cdots\left(m_{2}-m_{R}(\mathbf{s})+1\right) \cdot \prod_{\alpha, \beta \geq 0} \mathbb{E}\left\{G^{\alpha}\left(G^{2}-1\right)^{\beta}\right\}^{m_{\alpha, \beta}(\mathbf{s})} .
\end{aligned}
$$

Provided that $m_{1}=\left\lceil\sigma_{C}^{2} \vee 1\right\rceil+q-1$ and $m_{2}=\left\lceil\sigma_{R}^{2} \vee 1\right\rceil+q-1$, we have

$\sigma_{C}^{2 m_{L}(\mathbf{s})-2} \leq \frac{m_{1}\left(m_{1}-1\right) \cdots\left(m_{1}-m_{L}(\mathbf{s})+1\right)}{m_{1}}, \quad \sigma_{R}^{2 m_{R}(\mathbf{s})} \leq m_{2}\left(m_{1}-2\right) \cdots\left(m_{2}-m_{L}(\mathbf{s})+1\right) ;$

$\sigma_{C}^{2 m_{L}(\mathbf{s})} \leq m_{1}\left(m_{1}-1\right) \cdots\left(m_{1}-m_{L}(\mathbf{s})+1\right), \quad \sigma_{R}^{2 m_{R}(\mathbf{s})-2} \leq \frac{m_{2}\left(m_{1}-2\right) \cdots\left(m_{2}-m_{L}(\mathbf{s})+1\right)}{m_{2}}$.

Thus

$$
\mathbb{E} \operatorname{tr}\left\{\left(Z Z^{\top}-\mathbb{E} Z Z^{\top}\right)^{q}\right\} \leq\left(\frac{p_{1}}{m_{1}} \wedge \frac{p_{2}}{m_{2}}\right) \cdot \mathbb{E} \operatorname{tr}\left\{\left(H H^{\top}-\mathbb{E} H H^{\top}\right)^{q}\right\},
$$

which has finished the proof of this lemma.

Assume $B=1$ without loss of generality. With Lemma 5.4 and Lemma 2.6, the proof of Theorem refth:heter-wishart-bounded is the same as Theorem 2.1.

\subsection{Proof for tail bounds}

Proof of Theorem 3.8. Without loss of generality, we assume $\sigma_{*}=1$. Let $q \geq 2$ be an even integer. Let

$$
m_{1}=\left\lceil\sigma_{C}^{2}\right\rceil+q b-1, \quad m_{2}=\left\lceil\sigma_{R}^{2}\right\rceil+q b-1 .
$$

By Lemmas 2.4 and 2.6,

$$
\begin{aligned}
& \operatorname{Etr}\left\{\left(Z Z^{\top}-\mathbb{E} Z Z^{\top}\right)^{q b}\right\} \leq\left(\frac{p_{1}}{m_{1}} \wedge \frac{p_{2}}{m_{2}}\right) \operatorname{Etr}\left(\left(H H^{\top}-\mathbb{E} H H^{\top}\right)^{q b}\right) \\
\leq & \left(\frac{p_{1}}{m_{1}} \wedge \frac{p_{2}}{m_{2}}\right)\left(m_{1} \wedge m_{2}\right)\left(2 \sqrt{m_{1} m_{2}}+m_{1}+4\left(\sqrt{m_{1}}+\sqrt{m_{2}}\right) \sqrt{q b}+2 q b\right)^{q b} \\
\leq & \left(p_{1} \wedge p_{2}\right)\left(2 \sqrt{m_{1} m_{2}}+m_{1}+4\left(\sqrt{m_{1}}+\sqrt{m_{2}}\right) \sqrt{q b}+2 q b\right)^{q b} .
\end{aligned}
$$

Thus,

$$
\begin{aligned}
& \mathbb{E}\left\|Z Z^{\top}-\mathbb{E} Z Z^{\top}\right\|^{b} \leq\left(\mathbb{E} \operatorname{tr}\left(Z Z^{\top}-\mathbb{E} Z Z^{\top}\right)^{q b}\right)^{1 / q} \\
\leq & \left(p_{1} \wedge p_{2}\right)^{1 / q}\left(2 \sqrt{m_{1} m_{2}}+m_{1}+4\left(\sqrt{m_{1}}+\sqrt{m_{2}}\right) \sqrt{q b}+2 q b\right)^{b} \\
= & \left\{\left(p_{1} \wedge p_{2}\right)^{1 /(q b)}\left(2 \sqrt{m_{1} m_{2}}+m_{1}+4\left(\sqrt{m_{1}}+\sqrt{m_{2}}\right) \sqrt{q b}+2 q b\right)\right\}^{b} \\
\leq & \left\{C\left(p_{1} \wedge p_{2}\right)^{1 /(q b)}\left(\sigma_{R} \sigma_{C}+\sigma_{C}^{2}+\left(\sigma_{R}+\sigma_{C}\right) \sqrt{q b}+q b\right)\right\}^{b} .
\end{aligned}
$$

We set $q=2\left\lceil\log \left(p_{1} \wedge p_{2}\right) / b\right\rceil$ and consider the following two cases:

1. If $b \geq \log \left(p_{1} \wedge p_{2}\right)$, we have $q=2$ and

$$
\begin{aligned}
\mathbb{E}\left\|Z Z^{\top}-\mathbb{E} Z Z^{\top}\right\|^{b} & \leq\left\{C\left(p_{1} \wedge p_{2}\right)^{1 /(2 b)}\left(\sigma_{R} \sigma_{C}+\sigma_{C}^{2}+\left(\sigma_{R}+\sigma_{C}\right) \sqrt{b}+b\right)\right\}^{b} \\
& \leq\left\{C\left(\left(\sigma_{C}+\sigma_{R}+\sqrt{b}\right)^{2}-\sigma_{R}^{2}\right)\right\}^{b}
\end{aligned}
$$


2. If $b<\log \left(p_{1} \wedge p_{2}\right)$, we have

$$
2 \log \left(p_{1} \wedge p_{2}\right) / b \leq q=2\left\lceil\log \left(p_{1} \wedge p_{2}\right) / b\right\rceil \leq 2\left(\log \left(p_{1} \wedge p_{2}\right) / b+1\right) \leq 4 \log \left(p_{1} \wedge p_{2}\right) / b
$$

Then,

$$
\begin{aligned}
& \mathbb{E}\left\|Z Z^{\top}-\mathbb{E} Z Z^{\top}\right\|^{b} \\
\leq & \left\{C\left(p_{1} \wedge p_{2}\right)^{1 /\left(2 \log \left(p_{1} \wedge p_{2}\right)\right)}\right. \\
& \left.\left(\sigma_{R} \sigma_{C}+\sigma_{C}^{2}+\left(\sigma_{R}+\sigma_{C}\right) \sqrt{4 \log \left(p_{1} \wedge p_{2}\right)}+4 \log \left(p_{1} \wedge p_{2}\right)\right)\right\}^{b} \\
\leq & \left\{C\left(\left(\sigma_{C}+\sigma_{R}+\sqrt{\log \left(p_{1} \wedge p_{2}\right)}\right)^{2}-\sigma_{C}^{2}\right)\right\}^{b} .
\end{aligned}
$$

In summary, there exists a uniform constant $C_{0}>0$ such that

$$
\mathbb{E}\left\|Z Z^{\top}-\mathbb{E} Z Z^{\top}\right\|^{b} \leq\left\{C_{0}\left(\left(\sigma_{C}+\sigma_{R}+\sqrt{b \vee \log \left(p_{1} \wedge p_{2}\right)}\right)^{2}-\sigma_{C}^{2}\right)\right\}^{b} .
$$

In fact, the statement holds for all $b>0$ including non-integers.

Next we consider the tail bound inequality for $\left\|Z Z^{\top}-\mathbb{E} Z Z^{\top}\right\|$. Let $C_{1}$ be a to-bespecified constant. By Markov inequality,

$$
\begin{aligned}
& \mathbb{P}\left(\left\|Z Z^{\top}-\mathbb{E} Z Z^{\top}\right\| \geq C_{1}\left(\left(\sigma_{C}+\sigma_{R}+\sqrt{\log \left(p_{1} \wedge p_{2}\right)}+x\right)^{2}-\sigma_{C}^{2}\right)\right) \\
\leq & \frac{\mathbb{E}\left\|Z Z^{\top}-\mathbb{E} Z Z^{\top}\right\|^{b}}{\left\{C_{1}\left(\left(\sigma_{C}+\sigma_{R}+\sqrt{\log \left(p_{1} \wedge p_{2}\right)}+x\right)^{2}-\sigma_{C}^{2}\right)\right\}^{b}} \\
\leq & \left\{\frac{C_{0}\left(\left(\sigma_{C}+\sigma_{R}+\sqrt{b \vee \log \left(p_{1} \wedge p_{2}\right)}\right)^{2}-\sigma_{C}^{2}\right)}{C_{1}\left(\left(\sigma_{C}+\sigma_{R}+\sqrt{\log \left(p_{1} \wedge p_{2}\right)}+x\right)^{2}-\sigma_{C}^{2}\right)}\right\}^{b} .
\end{aligned}
$$

We set $b=x^{2}, C_{1}=e C_{0}$, we have

$$
\begin{aligned}
& \mathbb{P}\left(\left\|Z Z^{\top}-\mathbb{E} Z Z^{\top}\right\| \geq C_{1}\left(\left(\sigma_{C}+\sigma_{R}+\sqrt{\log \left(p_{1} \wedge p_{2}\right)}+x\right)^{2}-\sigma_{C}^{2}\right)\right) \\
\leq & \left\{\frac{C_{0}\left(\left(\sigma_{C}+\sigma_{R}+\sqrt{\log \left(p_{1} \wedge p_{2}\right)}+\sqrt{b}\right)^{2}-\sigma_{C}^{2}\right)}{C_{1}\left(\left(\sigma_{C}+\sigma_{R}+\sqrt{\log \left(p_{1} \wedge p_{2}\right)}+x\right)^{2}-\sigma_{C}^{2}\right)}\right\}^{b}=\exp \left(-x^{2}\right) .
\end{aligned}
$$

Therefore, we have finished the proof of this theorem.

\subsection{Proofs for Section 3.3}

Proof of Lemma 3.12. The proof of this lemma relies on a more careful counting scheme for each cycle. For convenience, we define

$$
\begin{gathered}
\tilde{\sigma}_{i j}^{2}=\operatorname{Var}\left(\tilde{G}_{i j}\right)= \begin{cases}\sigma_{i j}^{2}, & 1 \leq i \leq p_{1}-2,1 \leq j \leq p_{2} ; \\
\sigma_{p_{1}-1, j}^{2}+\sigma_{p_{1}, j}^{2}, & i=p-1,1 \leq j \leq p_{2} .\end{cases} \\
\left(G_{0}\right)_{i j}=G_{i j} / \sigma_{i j}, 1 \leq i \leq p_{2}, 1 \leq j \leq p_{1} ; \quad\left(\tilde{G}_{0}\right)_{i j}=\tilde{G}_{i j} / \tilde{\sigma}_{i j}, 1 \leq i \leq p_{1}-1,1 \leq j \leq p_{2}
\end{gathered}
$$

as the variances and standardizations of each entry of $G$ and $\tilde{G}$. Since the proof is lengthy, we divide into steps for a better presentation. 
Step 1. In this step, we consider the expansions for both $\operatorname{Etr}\left(G G^{\top}-\mathbb{E} G G^{\top}\right)^{q}$ and $\operatorname{Etr}\left(\tilde{G} \tilde{G}^{\top}-\mathbb{E} \tilde{G} \tilde{G}^{\top}\right)^{q}$,

$$
\begin{aligned}
& \mathbb{E} \operatorname{tr}\left\{\left(G G^{\top}-\mathbb{E} G G^{\top}\right)^{q}\right\}=\mathbb{E} \sum_{u_{1}, \ldots, u_{q} \in\left[p_{1}\right]} \prod_{k=1}^{q}\left(G G^{\top}-\mathbb{E} G G^{\top}\right)_{u_{k}, u_{k+1}} \\
= & \sum_{u_{1}, \ldots, u_{q} \in\left[p_{1}\right]} \mathbb{E} \prod_{k=1}^{q} \sigma_{u_{k}, v_{k}} \sigma_{u_{k+1}, v_{k}}\left(\left(G_{0}\right)_{u_{k}, v_{k}}\left(G_{0}\right)_{u_{k+1}, v_{k}}-1_{\left\{u_{k}=u_{k+1}\right\}}\right) \\
= & \sum_{\Omega \subseteq[q]} \sum_{u_{\Omega^{c}} \in\left[p_{1}-2\right]} \sum_{v_{1}, \ldots, v_{q} \in\left[p_{2}\right]} \\
& \left\{\sum_{u_{\Omega} \in\left\{p_{1}-1, p_{1}\right\}} \mathbb{E} \prod_{k=1}^{q} \sigma_{u_{k}, v_{k}} \sigma_{u_{k+1}, v_{k}}\left(\left(G_{0}\right)_{u_{k}, v_{k}}\left(G_{0}\right)_{u_{k+1}, v_{k}}-1_{\left\{u_{k}=u_{k+1}\right\}}\right)\right\} .
\end{aligned}
$$

Here $u_{q+1}:=u_{1}$. Similarly,

$$
\begin{aligned}
& \mathbb{E} \operatorname{tr}\left\{\left(\tilde{G} \tilde{G}^{\top}-\mathbb{E} \tilde{G} \tilde{G}^{\top}\right)^{q}\right\}=\mathbb{E} \sum_{u_{1}, \ldots, u_{q} \in\left[p_{1}-1\right]} \prod_{k=1}^{q}\left(\tilde{G} \tilde{G}^{\top}-\mathbb{E} \tilde{G} \tilde{G}^{\top}\right)_{u_{k}, u_{k+1}} \\
= & \sum_{\Omega \subseteq[q]} \sum_{u_{\Omega}} \in\left[p_{1}-2\right] \\
& \cdot\left\{\sum_{v_{1}, \ldots, v_{q} \in\left[p_{2}\right]} \mathbb{E} \prod_{k=1}^{q} \tilde{\sigma}_{u_{k}, v_{k}} \tilde{\sigma}_{u_{k+1}, v_{k}}\left(\left(\tilde{G}_{0}\right)_{u_{k}, v_{k}}\left(\tilde{G}_{0}\right)_{u_{k+1}, v_{k}}-1_{\left\{u_{k}=u_{k+1}\right\}}\right)\right\} .
\end{aligned}
$$

Thus, in order prove this lemma, we only need show for any fixed $v_{1}, \ldots, v_{q} \in\left[p_{2}\right]$, $\Omega \subseteq[q], u_{\Omega^{c}} \in\left[p_{1}-2\right]$, one has

$$
\begin{aligned}
& \sum_{u_{\Omega} \in\left\{p_{1}-1, p_{2}\right\}} \mathbb{E} \prod_{k=1}^{q} \sigma_{u_{k}, v_{k}} \sigma_{u_{k+1}, v_{k}}\left(\left(G_{0}\right)_{u_{k}, v_{k}}\left(G_{0}\right)_{u_{k+1}, v_{k}}-1_{\left\{u_{k}=u_{k+1}\right\}}\right) \\
\leq & \mathbb{E} \prod_{k=1}^{q} \tilde{\sigma}_{\tilde{u}_{k}, v_{k}} \tilde{\sigma}_{\tilde{u}_{k+1}, v_{k}}\left(\left(\tilde{G}_{0}\right)_{\tilde{u}_{k}, v_{k}}\left(\tilde{G}_{0}\right)_{\tilde{u}_{k+1}, v_{k}}-1_{\left\{\tilde{u}_{k}=\tilde{u}_{k+1}\right\}}\right) .
\end{aligned}
$$

Here,

$$
\tilde{u}_{k}=u_{k} \in\left[p_{1}-2\right], \text { if } k \in \Omega^{c} ; \quad \tilde{u}_{k}=p_{1}-1, \text { if } k \in \Omega .
$$

Step 2. To prove (5.29), we shall first recall that the definition of $u_{1}, \ldots, u_{q}, v_{1}, \ldots, v_{q}$ are cyclic, i.e., $u_{1}=u_{q+1}$, we also denote $v_{0}=v_{q}$. Thus,

$$
\begin{aligned}
& \sum_{u_{\Omega} \in\left\{p_{1}-1, p_{1}\right\}} \prod_{k=1}^{q} \sigma_{u_{k}, v_{k}} \sigma_{u_{k+1}, v_{k}}=\sum_{u_{\Omega} \in\left\{p_{1}-1, p_{1}\right\}} \prod_{k=1}^{q} \sigma_{u_{k}, v_{k}} \sigma_{u_{k}, v_{k-1}} \\
& =\prod_{k \in \Omega^{c}} \sigma_{u_{k}, v_{k}} \sigma_{u_{k}, v_{k-1}} \cdot\left(\prod_{k \in \Omega} \sigma_{p_{1}-1, v_{k}} \sigma_{p_{1}-1, v_{k-1}}+\prod_{k \in \Omega} \sigma_{p_{1}, v_{k}} \sigma_{p_{1}, v_{k-1}}\right) \\
& \leq \prod_{k \in \Omega^{c}} \sigma_{u_{k}, v_{k}} \sigma_{u_{k}, v_{k-1}} \cdot \prod_{k \in \Omega}\left(\sigma_{p_{1}-1, v_{k}} \sigma_{p_{1}-1, v_{k-1}}+\sigma_{p_{1}, v_{k}} \sigma_{p_{1}, v_{k-1}}\right) \\
& \stackrel{\text { Cauchy-Schwarz }}{\leq} \prod_{k \in \Omega^{c}} \tilde{\sigma}_{u_{k}, v_{k}} \tilde{\sigma}_{u_{k}, v_{k-1}} \cdot \prod_{k \in \Omega}\left(\left(\sigma_{p_{1}-1, v_{k}}^{2}+\sigma_{p_{1}, v_{k}}^{2}\right) \cdot\left(\sigma_{p_{1}-1, v_{k-1}}^{2}+\sigma_{p_{1}, v_{k-1}}^{2}\right)\right)^{1 / 2} \\
& =\prod_{k \in \Omega^{c}} \tilde{\sigma}_{u_{k}, v_{k}} \tilde{\sigma}_{u_{k}, v_{k-1}} \cdot \prod_{k \in \Omega} \tilde{\sigma}_{p_{1}-1, v_{k}} \tilde{\sigma}_{p_{1}-1, v_{k-1}}
\end{aligned}
$$




$$
=\sum_{u_{\Omega}=p_{1}-1} \prod_{k=1}^{q} \tilde{\sigma}_{u_{k}, v_{k}} \tilde{\sigma}_{u_{k}, v_{k-1}}=\sum_{u_{\Omega}=p_{1}-1} \prod_{k=1}^{q} \tilde{\sigma}_{u_{k}, v_{k}} \tilde{\sigma}_{u_{k+1}, v_{k}} .
$$

Step 3. For any fixed $\Omega=\left\{k: u_{k} \in\left[p_{1}-2\right]\right\}$ and a cycle $\mathbf{c}=\left(u_{1} \rightarrow v_{1} \rightarrow u_{2} \rightarrow v_{2} \rightarrow \ldots \rightarrow\right.$ $\left.u_{q} \rightarrow v_{q} \rightarrow u_{1}\right)$ such that $u_{\Omega} \in\left\{p_{1}-1, p\right\}$ and $u_{\Omega} \in\left[p_{1}-2\right]$, recall $\tilde{u}_{k}$ is defined as (5.30). We aim to show in this step that

$$
\mathbb{E} \prod_{k=1}^{q}\left(\left(G_{0}\right)_{u_{k}, v_{k}}\left(G_{0}\right)_{u_{k+1}, v_{k}}-1_{\left\{u_{k}=u_{k+1}\right\}}\right) \leq \mathbb{E} \prod_{k=1}^{q}\left(\left(\tilde{G}_{0}\right)_{\tilde{u}_{k}, v_{k}}\left(\tilde{G}_{0}\right)_{\tilde{u}_{k+1}, v_{k}}-1_{\left\{\tilde{u}_{k}=\tilde{u}_{k+1}\right\}}\right) .
$$

We can rearrange the left hand side and the right hand side of (5.32) to

$$
\mathbb{E} \prod_{i=1}^{p_{1}} \prod_{j=1}^{p_{2}}\left(G_{0}\right)_{i j}^{\alpha_{i j}}\left(\left(G_{0}\right)_{i j}^{2}-1\right)^{\beta_{i j}}, \quad \text { and } \mathbb{E} \prod_{i=1}^{p_{1}-1} \prod_{j=1}^{p_{2}}\left(\tilde{G}_{0}\right)_{i j}^{\tilde{\alpha}_{i j}}\left(\left(\tilde{G}_{0}\right)_{i j}^{2}-1\right)^{\tilde{\beta}_{i j}} .
$$

Here, $\alpha_{i j}, \beta_{i j}, \tilde{\alpha}_{i j}$, and $\tilde{\beta}_{i j}$ are defined as

$$
\begin{aligned}
\alpha_{i j} & =\mid\left\{k:\left(u_{k}=i, v_{k}=j, u_{k+1} \neq i\right) \text { or }\left(u_{k} \neq i, v_{k}=j, u_{k+1}=i\right)\right\} \mid, \\
\beta_{i j} & =\left|\left\{k: u_{k}=u_{k+1}=i, v_{k}=j\right\}\right|, \\
\tilde{\alpha}_{i j} & =\mid\left\{k:\left(\tilde{u}_{k}=i, v_{k}=j, \tilde{u}_{k+1} \neq i\right) \text { or }\left(\tilde{u}_{k} \neq i, v_{k}=j, \tilde{u}_{k+1}=i\right)\right\} \mid \\
\tilde{\beta}_{i j} & =\left|\left\{k: \tilde{u}_{k}=\tilde{u}_{k+1}=i, v_{k}=j\right\}\right| .
\end{aligned}
$$

Then, $\alpha_{i j}$ (or $\tilde{\alpha}_{i j}$ ) is the number of times that the edge $(i, j)$ is visited exactly once by sub-path $u_{k} \rightarrow v_{k} \rightarrow u_{k+1}$ (or $\tilde{u}_{k} \rightarrow v_{k} \rightarrow \tilde{u}_{k+1}$ ); $\beta_{i j}$ (or $\tilde{\beta}_{i j}$ ) is the number of times that the edge $(i, j)$ is visited twice (back and forth) by sub-path $u_{k} \rightarrow v_{k} \rightarrow u_{k+1}$ (or $\left.\tilde{u}_{k} \rightarrow v_{k} \rightarrow \tilde{u}_{k+1}\right)$.

Here, by comparing the order of $\left(\tilde{G}_{0}\right)_{i j}$ and $\left(G_{0}\right)_{i j}$ in these two monomials (5.32), $\tilde{\alpha}_{i j}, \tilde{\beta}_{i j}, \alpha_{i j}, \beta_{i j}$ are related as

$$
\tilde{\alpha}_{i j}=\alpha_{i j}, \quad \tilde{\beta}_{i j}=\beta_{i j}, \quad \text { if } 1 \leq i \leq p_{1}-2,1 \leq j \leq n,
$$

The relationship among $\tilde{\alpha}_{p_{1}-1, j}, \tilde{\beta}_{p_{1}-1, j}, \alpha_{p_{1}-1, j}, \alpha_{p_{1}, j}, \beta_{p_{1}-1, j}, \beta_{p_{1}, j}$ is more involved. To analyze them, for any fixed $1 \leq j \leq p_{2}$ we define

$$
\begin{aligned}
& x_{1}^{(j)}=\mid\left\{k:\left(u_{k} \rightarrow v_{k} \rightarrow u_{k+1}\right)=\left(\left(p_{1}-1\right) \rightarrow j \rightarrow\left\{p_{1}-1, p_{1}\right\}^{c}\right)\right. \\
& \left.\quad \text { or }\left(\left\{p_{1}-1, p_{1}\right\}^{c} \rightarrow j \rightarrow\left(p_{1}-1\right)\right)\right\} \mid, \\
& x_{2}^{(j)}=\mid\left\{k:\left(u_{k} \rightarrow v_{k} \rightarrow u_{k+1}\right)=\left(p_{1} \rightarrow j \rightarrow\left\{p_{1}-1, p_{1}\right\}^{c}\right) \text { or }\left(\left\{p_{1}-1, p_{1}\right\}^{c} \rightarrow j \rightarrow p_{1}\right)\right\} \mid, \\
& x_{3}^{(j)}=\left|\left\{k:\left(u_{k} \rightarrow v_{k} \rightarrow u_{k+1}\right)=\left(\left(p_{1}-1\right) \rightarrow j \rightarrow\left(p_{1}-1\right)\right)\right\}\right|, \\
& x_{4}^{(j)}=\left|\left\{k:\left(u_{k} \rightarrow v_{k} \rightarrow u_{k+1}\right)=\left(p_{1} \rightarrow j \rightarrow p_{1}\right)\right\}\right|, \\
& x_{5}^{(j)}=\mid\left\{k:\left(u_{k} \rightarrow v_{k} \rightarrow u_{k+1}\right)=\left(\left(p_{1}-1\right) \rightarrow j \rightarrow p_{1}\right) \text { or }\left(p_{1} \rightarrow j \rightarrow\left(p_{1}-1\right)\right)\right\} \mid .
\end{aligned}
$$

Then by definitions, we have

$$
\begin{aligned}
& \alpha_{p_{1}-1, j}=x_{1}^{(j)}+x_{5}^{(j)}, \quad \alpha_{p_{1}, j}=x_{2}^{(j)}+x_{5}^{(j)}, \quad \tilde{\alpha}_{p_{1}-1, j}=x_{1}^{(j)}+x_{2}^{(j)} ; \\
& \beta_{p_{1}-1, j}=x_{3}^{(j)}, \quad \beta_{p_{1}, j}=x_{4}^{(j)}, \quad \tilde{\beta}_{p_{1}-1, j}=x_{3}^{(j)}+x_{4}^{(j)}+x_{5}^{(j)} .
\end{aligned}
$$

We introduce the following Lemma before we proceed.

Lemma 5.5. Suppose $Z_{1}, Z_{2}$ are independent and symmetric distributed random variables. $\operatorname{Var}\left(Z_{1}\right)=\operatorname{Var}\left(Z_{2}\right)=1,\left\|Z_{1}\right\|_{\psi_{2}},\left\|Z_{2}\right\|_{\psi_{2}} \leq \kappa$. $G$ is standard Gaussian distributed. For any non-negative integers $x_{1}, \ldots, x_{5}$, we have

$$
\begin{aligned}
& \left|\mathbb{E} Z_{1}^{x_{1}+x_{5}} Z_{2}^{x_{2}+x_{5}}\left(Z_{1}^{2}-1\right)^{x_{3}}\left(Z_{2}^{2}-1\right)^{x_{4}}\right| \\
& \leq(C \kappa)^{x_{1}+x_{2}+2\left(x_{3}+x_{4}+x_{5}\right)} \mathbb{E} G^{x_{1}+x_{2}}\left(G^{2}-1\right)^{x_{3}+x_{4}+x_{5}} \\
& \quad \leq
\end{aligned}
$$


Especially when $Z_{1}, Z_{2}, G$ are all standard Gaussian,

$$
\left|\mathbb{E} Z_{1}^{x_{1}+x_{5}} Z_{2}^{x_{2}+x_{5}}\left(Z_{1}^{2}-1\right)^{x_{3}}\left(Z_{2}^{2}-1\right)^{x_{4}}\right| \leq \mathbb{E} G^{x_{1}+x_{2}}\left(G^{2}-1\right)^{x_{3}+x_{4}+x_{5}} .
$$

Proof. See Appendix.

By Lemma 5.5,

$$
\begin{aligned}
& \left|\mathbb{E}\left(G_{0}\right)_{p_{1}-1, j}^{\alpha_{p_{1}-1, j}}\left(\left(G_{0}\right)_{p_{1}-1, j}^{2}-1\right)^{\beta_{p_{1}-1, j}}\right| \cdot\left|\mathbb{E}\left(G_{0}\right)_{p_{1}, j}^{\alpha_{p_{1}, j}}\left(\left(G_{0}\right)_{p_{1}, j}^{2}-1\right)^{\beta_{p_{1}, j}}\right| \\
= & \left|\mathbb{E}\left(G_{0}\right)_{p_{1}-1, j}^{x_{1}^{(j)}+x_{5}^{(j)}}\left(\left(G_{0}\right)_{p_{1}-1, j}^{2}-1\right)^{x_{3}^{(j)}}\right| \cdot\left|\mathbb{E}\left(G_{0}\right)_{p_{1}, j}^{x_{2}^{(j)}+x_{5}^{(j)}}\left(\left(G_{0}\right)_{p_{1}, j}^{2}-1\right)^{x_{4}^{(j)}}\right| \\
\leq & \mathbb{E}\left(\tilde{G}_{0}\right)_{p_{1}-1, j}^{x_{1}^{(j)}+x_{2}^{(j)}}\left(\left(\tilde{G}_{0}\right)_{p_{1}-1, j}^{2}-1\right)^{x_{3}^{(j)}+x_{4}^{(j)}+x_{5}^{(j)}}=\mathbb{E}\left(\tilde{G}_{0}\right)_{p_{1}-1, j}^{\tilde{\alpha}_{p_{1}-1, j}}\left(\left(\tilde{G}_{0}\right)_{p_{1}-1, j}^{2}-1\right)^{\tilde{\beta}_{p_{1}-1, j}} .
\end{aligned}
$$

Thus,

$$
\mathbb{E} \prod_{i=1}^{p_{1}} \prod_{j=1}^{p_{2}}\left(G_{0}\right)_{i j}^{\alpha_{i j}}\left(\left(G_{0}\right)_{i j}^{2}-1\right)^{\beta_{i j}} \leq \mathbb{E} \prod_{i=1}^{p_{1}-1} \prod_{j=1}^{p_{2}}\left(\tilde{G}_{0}\right)_{i j}^{\tilde{\alpha}_{i j}}\left(\left(\tilde{G}_{0}\right)_{i j}^{2}-1\right)^{\tilde{\beta}_{i j}} .
$$

This gives (5.32).

Step 4. Combining (5.31) and (5.32), we finally have

$$
\begin{aligned}
& \sum_{u_{\Omega} \in\left\{p_{1}-1, p_{1}\right\}} \mathbb{E} \prod_{k=1}^{q} \sigma_{u_{k}, v_{k}} \sigma_{u_{k+1}, v_{k}}\left(\left(G_{0}\right)_{u_{k}, v_{k}}\left(G_{0}\right)_{u_{k+1}, v_{k}}-1_{\left\{u_{k}=u_{k+1}\right\}}\right) \\
&= \sum_{u_{\Omega} \in\left\{p_{1}-1, p_{1}\right\}} \prod_{k=1}^{q} \sigma_{u_{k}, v_{k}} \sigma_{u_{k+1}, v_{k}} \cdot \mathbb{E} \prod_{k=1}^{q}\left(\left(G_{0}\right)_{u_{k}, v_{k}}\left(G_{0}\right)_{u_{k+1}, v_{k}}-1_{\left\{u_{k}=u_{k+1}\right\}}\right) \\
& \stackrel{(5.32)}{\leq} \sum_{u_{\Omega} \in\left\{p_{1}-1, p_{1}\right\}} \prod_{k=1}^{q} \sigma_{u_{k}, v_{k}} \sigma_{u_{k+1}, v_{k}} \cdot \mathbb{E} \prod_{k=1}^{q}\left(\left(\tilde{G}_{0}\right)_{\tilde{u}_{k}, v_{k}}\left(\tilde{G}_{0}\right)_{\tilde{u}_{k+1}, v_{k}}-1_{\left\{\tilde{u}_{k}=\tilde{u}_{k+1}\right\}}\right) \\
& \stackrel{(5.31)}{\leq} \mathbb{E} \prod_{k=1}^{q} \tilde{\sigma}_{\tilde{u}_{k}, v_{k}} \tilde{\sigma}_{\tilde{u}_{k+1}, v_{k}}\left(\left(\tilde{G}_{0}\right)_{\tilde{u}_{k}, v_{k}}\left(\tilde{G}_{0}\right)_{\tilde{u}_{k+1}, v_{k}}-1_{\left\{\tilde{u}_{k}=\tilde{u}_{k+1}\right\}}\right),
\end{aligned}
$$

which yields (5.29) and additionally finishes the proof of this lemma.

Proof of Theorem 3.11. Denote $\sigma_{C}^{2}=\sum_{i} \sigma_{i}^{2}, \sigma_{*}=\max _{i} \sigma_{i}, Z=\left[Z_{1}, \ldots, Z_{p_{2}}\right]$, and $S_{k}=$ $Z_{k} Z_{k}^{\top}-\mathbb{E} Z_{k} Z_{k}^{\top}$. Then

$$
\mathbb{E}\left\|Z Z^{\top}-\mathbb{E} Z Z^{\top}\right\|=\mathbb{E}\left\|\sum_{k=1}^{p_{2}} S_{k}\right\| .
$$

By the lower bound for expected norm of independent random matrices sum [29, Theorem I and Section 1.3],

$$
\mathbb{E}\left\|Z Z^{\top}-\mathbb{E} Z Z^{\top}\right\| \gtrsim\left(\left\|\mathbb{E} \sum_{k=1}^{p_{2}} S_{k} S_{k}^{\top}\right\|\right)^{1 / 2}+\mathbb{E} \max _{k}\left\|S_{k}\right\| .
$$

If $Z_{i j} \sim N\left(0, \sigma_{i}^{2}\right)$ for any $i \in\left[p_{1}\right], j \in\left[p_{2}\right]$. Note that

$$
\begin{aligned}
& \left(\mathbb{E} Z_{k} Z_{k}^{\top} Z_{k} Z_{k}^{\top}\right)_{i j}=\mathbb{E} Z_{i k} \sum_{l=1}^{p_{1}} Z_{l k}^{2} Z_{j k} \\
& =\left\{\begin{array}{ll}
3 \sigma_{i}^{4}+\sigma_{i}^{2}\left(\sum_{l \neq i} \sigma_{l}^{2}\right), & i=j ; \\
0, & i \neq j,
\end{array}=\operatorname{diag}\left(\left\{2 \sigma_{i}^{4}+\sigma_{i}^{2} \sigma_{C}^{2}\right\}_{i=1}^{p_{1}}\right)\right. \\
& \left(\mathbb{E} Z_{k} Z_{k}^{\top}\right)^{2}=\operatorname{diag}\left(\sigma_{1}^{4}, \ldots, \sigma_{p_{1}}^{4}\right) \text {. }
\end{aligned}
$$


Thus,

$$
\begin{aligned}
\left\|\mathbb{E} \sum_{k=1}^{p_{2}} S_{k} S_{k}^{\top}\right\| & =\left\|\sum_{k=1}^{p_{2}} \mathbb{E}\left(Z_{k} Z_{k}^{\top}-\mathbb{E} Z_{k} Z_{k}^{\top}\right)\left(Z_{k} Z_{k}^{\top}-\mathbb{E} Z_{k} Z_{k}^{\top}\right)\right\| \\
& =\left\|\sum_{k=1}^{p_{2}} \mathbb{E} Z_{k} Z_{k}^{\top} Z_{k} Z_{k}^{\top}-\left(\mathbb{E} Z_{k} Z_{k}^{\top}\right)^{2}\right\| \\
& =\left\|\operatorname{diag}\left(\left\{\sigma_{i}^{2} \sigma_{C}^{2}+\sigma_{i}^{4}\right\}_{i=1}^{p_{1}}\right)\right\|=\sigma_{*}^{4}+\sigma_{*}^{2} \sigma_{C}^{2} .
\end{aligned}
$$

Meanwhile, let $i^{*} \in[p]$ such that suppose $\sigma_{*}=\sigma_{i^{*}}$, then

$$
\begin{gathered}
\mathbb{E}\left\|S_{k}\right\|=\mathbb{E}\left\|Z_{k} Z_{k}^{\top}-\mathbb{E} Z_{k} Z_{k}^{\top}\right\| \geq \mathbb{E}\left\|Z_{k} Z_{k}^{\top}\right\|-\left\|\mathbb{E} Z_{k} Z_{k}^{\top}\right\|=\sigma_{C}^{2}-\sigma_{*}^{2} ; \\
\mathbb{E}\left\|S_{k}\right\| \geq \mathbb{E}\left\|\left(S_{k}\right)_{i^{*} i^{*}}\right\|=\mathbb{E}\left|Z_{i^{*} k}^{2}-\mathbb{E} Z_{i^{*} k}^{2}\right| \geq c \sigma_{*}^{2} .
\end{gathered}
$$

Combining the previous two inequalities, we have $\mathbb{E}\left\|S_{k}\right\| \geq c \sigma_{C}^{2}$. Consequently,

$$
\mathbb{E}\left\|Z Z^{\top}-\mathbb{E} Z Z^{\top}\right\| \stackrel{(5.36)}{\gtrsim} \sigma_{C}^{2}+\sqrt{p_{2}} \sigma_{*} \sigma_{C} .
$$

\subsection{Proofs for Section 3.4}

Proof of Lemma 3.15. Since the diagonal of $\Delta\left(Z Z^{\top}\right)$ is zero, we have the following expansion,

$$
\begin{aligned}
& \mathbb{E} \operatorname{tr}\left\{\left(\Delta\left(Z Z^{\top}\right)\right)^{q}\right\}=\sum_{u_{1}, \ldots, u_{1} \in\left[p_{1}\right]} \mathbb{E} \prod_{k=1}^{q}\left(\Delta\left(Z Z^{\top}\right)\right)_{u_{k}, u_{k+1}} \\
= & \sum_{\substack{u_{1}, \ldots, u_{1} \in\left[p_{1}\right] \\
v_{1}, \ldots v_{q} \in\left[p_{2}\right]}} \mathbb{E} \prod_{k=1}^{q}\left(1_{\left\{u_{k} \neq u_{k+1}\right\}} Z_{u_{k}, v_{k}} Z_{u_{k+1}, v_{k}}\right) .
\end{aligned}
$$

Again, the indices on $u$ are in module $q$, i.e., $u_{1}=u_{q+1}$. For a cycle $\mathbf{c}:=\left(u_{1} \rightarrow v_{1} \rightarrow u_{2} \rightarrow\right.$ $\left.v_{2} \rightarrow \ldots \rightarrow u_{q} \rightarrow v_{q} \rightarrow u_{1}\right)$, recall the definition of $\alpha_{i j}(\mathbf{c})$ :

$$
\alpha_{i j}(\mathbf{c})=\operatorname{Card}\left\{k:\left(u_{k}=i, v_{k}=j, u_{k+1} \neq i\right) \text { or }\left(u_{k} \neq i, v_{k}=j, u_{k+1}=i\right)\right\}
$$

for any $i \in\left[p_{1}\right]$ and $j \in\left[p_{2}\right]$, which counts how many times edge $i \rightarrow j$ or $j \rightarrow i$ are visited. Now the expansion in (5.37) can be further written as

$$
\begin{aligned}
& \mathbb{E} \operatorname{tr}\left\{\left(\Delta\left(Z Z^{\top}\right)\right)^{q}\right\}=\sum_{\mathbf{c} \in\left[\left[p_{1}\right] \times\left[p_{2}\right]\right]^{q}}\left(\prod_{k=1}^{q} 1_{\left\{u_{k} \neq u_{k+1}\right\}}\right) \cdot\left(\prod_{(i, j) \in\left[p_{1}\right] \times\left[p_{2}\right]} \mathbb{E} Z_{i j}^{\alpha_{i j}(\mathbf{c})}\right) \\
= & \sum_{\mathbf{c} \in\left(\left[p_{1}\right] \times\left[p_{2}\right]\right)^{q}}\left(\prod_{k=1}^{q} 1_{\left\{u_{k} \neq u_{k+1}\right\}} \sigma_{u_{k}, v_{k}} \sigma_{u_{k+1}, v_{k}}\right) \cdot\left(\prod_{(i, j) \in\left[p_{1}\right] \times\left[p_{2}\right]} \mathbb{E} G^{\alpha_{i j}(\mathbf{c})}\right) \\
= & \sum_{\mathbf{c} \in\left(\left[p_{1}\right] \times\left[p_{2}\right]\right)^{q}}\left(\prod_{k=1}^{q} 1_{\left\{u_{k} \neq u_{k+1}\right\}} \sigma_{v_{k}}^{2}\right) \cdot\left(\prod_{(i, j) \in\left[p_{1}\right] \times\left[p_{2}\right]} \mathbb{E} G^{\alpha_{i j}(\mathbf{c})}\right) .
\end{aligned}
$$

We define $m_{\alpha}(\mathbf{c})$ be the number of edges which appear $\alpha$ times in the cycle c:

$$
m_{\alpha}(\mathbf{c})=\operatorname{Card}\left\{(i, j) \in\left[p_{1}\right] \times\left[p_{2}\right]: \mid\left\{k: u_{k} \text { or } u_{k+1}=i, v_{k}=j,\right\} \mid=\alpha\right\}
$$

Let $\mathbf{s}(\mathbf{c})$ be the shape of $\mathbf{c}$, we have

$$
\prod_{(i, j) \in\left[p_{1}\right] \times\left[p_{2}\right]} \mathbb{E} G^{\alpha_{i j}(\mathbf{c})}=\prod_{\alpha \geq 0} \mathbb{E} G^{m_{\alpha}(\mathbf{s}(\mathbf{c}))},
$$


where $G \sim N(0,1)$. Next we define the following shape family:

$$
\mathcal{S}_{p_{1}, p_{2}}:=\left\{\mathbf{s}(\mathbf{c}): m_{\alpha}^{\prime}(\mathbf{c})=0 \text { for all odd } \alpha \text {; and } u_{k} \neq u_{k+1} \text { for all } k=1, \ldots, q\right\} \text {. }
$$

Based on the notations above, one can check the expansion in (5.38) can be further simplified to

$$
\begin{aligned}
\operatorname{Etr}\left\{\left(\Delta\left(Z Z^{\top}\right)\right)^{q}\right\} & =\sum_{\mathbf{s}_{0} \in \mathcal{S}_{p_{1}, p_{2}}} \sum_{\mathbf{c}: \mathbf{s}(\mathbf{c})=\mathbf{s}_{0}}\left(\prod_{k=1}^{q} \sigma_{v_{k}}^{2}\right) \prod_{\alpha \geq 0} \mathbb{E} G^{m_{\alpha}\left(\mathbf{s}_{0}\right)} \\
& =\sum_{\mathbf{s}_{0} \in \mathcal{S}_{p_{1}, p_{2}}} \prod_{\alpha \geq 0} \mathbb{E} G^{m_{\alpha}\left(\mathbf{s}_{0}\right)} \sum_{\mathbf{c}: \mathbf{s}(\mathbf{c})=\mathbf{s}_{0}}\left(\prod_{k=1}^{q} \sigma_{v_{k}}^{2}\right)
\end{aligned}
$$

For a fixed shape $\mathbf{s}_{0} \in \mathcal{S}_{p_{1}, p_{2}}$, let $m_{L}\left(\mathbf{s}_{0}\right)\left(m_{R}\left(\mathbf{s}_{0}\right)\right)$ be the number of distinct left (right) vertexes visited by cycles with shape $\mathbf{s}_{0}$. Now we bound $\sum_{\mathbf{c}: \mathbf{s}(\mathbf{c})=\mathbf{s}_{0}}\left(\prod_{k=1}^{q} \sigma_{v_{k}}^{2}\right)$ via $m_{L}\left(\mathbf{s}_{0}\right)$ and $m_{R}\left(\mathbf{s}_{0}\right)$. To this end, we first present three facts for any cycles with shape $\mathbf{s}_{0}$ :

- Each visited edges must appear at least twice in the cycles;

- For each right vertex in the cycle, its predecessor and successor in left vertex set must be different;

- The cycle is uniquely defined by specifying $m_{L}\left(\mathbf{s}_{0}\right)$ left vertexes and $m_{R}\left(\mathbf{s}_{0}\right)$ right vertexes; moreover, the summation term is free of the index of the left visited vertexes.

These three observations, together with the assumption $\sigma_{*}=1$, yield the following bound:

$$
\sum_{\mathbf{c}: \mathbf{s}(\mathbf{c})=\mathbf{s}_{0}}\left(\prod_{k=1}^{q} \sigma_{v_{k}}^{2}\right) \leq p_{1}\left(p_{1}-1\right) \cdots\left(p_{1}-m_{L}\left(\mathbf{s}_{0}\right)+1\right)\left(\sum_{j=1}^{n} \sigma_{j}^{4}\right)^{m_{R}\left(\mathbf{s}_{0}\right)} .
$$

Next we make comparison between $\operatorname{Etr}\left\{\left(\Delta\left(Z Z^{\top}\right)\right)^{q}\right\}$ and $\operatorname{Etr}\left\{\left(\Delta\left(H H^{\top}\right)\right)^{q}\right\}$, where $H$ is a $p_{1}$-by- $m$ random matrix with i.i.d. standard Gaussian entries. Similar, as above, we have

$$
\operatorname{Etr}\left\{\left(\Delta\left(H H^{\top}\right)\right)^{q}\right\}=\sum_{\mathbf{s}_{0} \in \mathcal{S}_{p_{1}, p_{2}}} \prod_{\alpha \geq 0} \mathbb{E} G^{m_{\alpha}\left(\mathbf{s}_{0}\right)} \sum_{\mathbf{c}: \mathbf{s}(\mathbf{c})=\mathbf{s}_{0}}\left|\left\{\mathbf{c}: \mathbf{s}(\mathbf{c})=\mathbf{s}_{0}\right\}\right|
$$

Setting $m=\left\lceil\sum_{j=1}^{p_{2}} \sigma_{j}^{4}\right\rceil+q-1$, we have

$$
\begin{aligned}
\left|\left\{\mathbf{c}: \mathbf{s}(\mathbf{c})=\mathbf{s}_{0}\right\}\right| & =p_{1}\left(p_{1}-1\right) \cdots\left(p_{1}-m_{L}\left(\mathbf{s}_{0}\right)+1\right) m(m-1) \cdots\left(m-m_{R}\left(\mathbf{s}_{0}\right)+1\right) \\
& \geq p_{1}\left(p_{1}-1\right) \cdots\left(p_{1}-m_{L}\left(\mathbf{s}_{0}\right)+1\right)\left(m-m_{R}\left(\mathbf{s}_{0}\right)+1\right)^{m_{R}\left(\mathbf{s}_{0}\right)} \\
& \geq p_{1}\left(p_{1}-1\right) \cdots\left(p_{1}-m_{L}\left(\mathbf{s}_{0}\right)+1\right)\left(\sum_{j=1}^{n} \sigma_{j}^{4}\right)^{m_{R}\left(\mathbf{s}_{0}\right)}
\end{aligned}
$$

Combining (5.40) and (5.41), we finish the proof.

Proof of Theorem 3.14. Denote $\sigma_{R}^{2}=\sum_{j} \sigma_{j}^{2}, \sigma_{*}=\max _{i} \sigma_{i}$. We use the general lower bound for expected norm of independent random matrices sum [29, Theorem I and Section 1.3] as we did in the proof of Theorem 3.11. Since $Z_{i j} \sim N\left(0, \sigma_{j}^{2}\right)$, for any 
$k \in\left[p_{2}\right]$,

$$
\begin{aligned}
& \left(\mathbb{E} Z_{k} Z_{k}^{\top} Z_{k} Z_{k}^{\top}\right)_{i j}=\mathbb{E} Z_{i k} \sum_{l=1}^{p_{1}} Z_{l k}^{2} Z_{j k} \\
& =\left\{\begin{array}{ll}
3 \sigma_{k}^{4}+\left(p_{1}-1\right) \sigma_{k}^{4}, & i=j ; \\
0, & i \neq j,
\end{array}=\operatorname{diag}\left(\left\{\left(p_{1}+2\right) \sigma_{k}^{2}\right\}_{i=1}^{p_{1}}\right)\right. \\
& \left(\mathbb{E} Z_{k} Z_{k}^{\top}\right)^{2}=\sigma_{k}^{4} I_{p_{1}}
\end{aligned}
$$

Thus,

$$
\left\|\mathbb{E} \sum_{k=1}^{p_{2}} S_{k} S_{k}^{\top}\right\|=\left\|\sum_{k=1}^{p_{2}} \mathbb{E} Z_{k} Z_{k}^{\top} Z_{k} Z_{k}^{\top}-\left(\mathbb{E} Z_{k} Z_{k}^{\top}\right)^{2}\right\|=\left\|\left(p_{1}+1\right)\left(\sum_{k=1}^{p_{2}} \sigma_{k}^{4}\right) I_{p_{1}}\right\| \geq p_{1} \sum_{k=1}^{p_{2}} \sigma_{k}^{4} .
$$

On the other hand,

$$
\mathbb{E} \max _{k}\left\|S_{k}\right\| \geq \max _{k} \mathbb{E}\left\|S_{k}\right\| \geq \max _{k}\left\{\mathbb{E}\left\|Z_{k} Z_{k}^{\top}\right\|-\left\|\mathbb{E} Z_{k} Z_{k}^{\top}\right\|\right\}=\left(p_{1}-1\right) \sigma_{*}^{2} .
$$

Combining the previous two inequalities and (5.36) in the proof of Theorem 3.11, we obtain

$$
\mathbb{E}\left\|Z Z^{\top}-\mathbb{E} Z Z^{\top}\right\| \stackrel{(5.36)}{\gtrsim} \sqrt{p_{1} \sum_{k=1}^{p_{2}} \sigma_{k}^{2}}+p_{1} \sigma_{*}^{2} .
$$

\subsection{Proofs for heteroskedastic clustering}

Proof of Theorem 4.1. We first introduce following three lemmas.

Lemma 5.6. For any $x \in\{-1,+1\}^{n}$ and $z \in \mathbb{R}$ with $\|z\|_{2}=1$ we have

$$
d(x, \operatorname{sgn}(z)) \leq n\left\|\frac{x}{\sqrt{n}}-z\right\|_{2}^{2} .
$$

Here $d$ represents the Hamming distance: $d(x, z)=\sum_{i=1}^{n} 1_{\left\{x_{i} \neq y_{i}\right\}}$.

Proof. See [21].

Lemma 5.7. Assume that $Z \in \mathbb{R}^{p_{1} \times p_{2}}$ has independent sub-Gaussian entries, $\operatorname{Var}\left(Z_{i j}\right)=$ $\sigma_{i j}^{2}, \sigma_{C}^{2}=\max _{j} \sum_{i} \sigma_{i j}^{2}, \sigma_{R}^{2}=\max _{i} \sum_{j} \sigma_{i j}^{2}, \sigma_{*}^{2}=\max _{i, j} \sigma_{i j}^{2}$. Assume that $\left\|Z_{i j} / \sigma_{i j}\right\|_{\psi_{2}} \leq \kappa$. Let $V \in \mathbb{O}_{p_{2}, r}$ be a fixed orthogonal matrix. Then,

$$
\begin{gathered}
\mathbb{P}\left(\|E V\| \geq 2\left(\sigma_{C}+x\right)\right) \leq 2 \exp \left(5 r-\min \left\{\frac{x^{4}}{\kappa^{4} \sigma_{*}^{2} \sigma_{C}^{2}}, \frac{x^{2}}{\kappa^{2} \sigma_{*}^{2}}\right\}\right), \\
\mathbb{E}\|E V\| \lesssim \sigma_{C}+\kappa r^{1 / 4}\left(\sigma_{*} \sigma_{C}\right)^{1 / 2}+\kappa r^{1 / 2} \sigma_{*} .
\end{gathered}
$$

Proof. See [36, Lemma 3].

Lemma 5.8 (Davis-Kahan). Let $A$ be an $n$-by-n symmetric matrix with eigenvalues $\left|\lambda_{1}\right| \geq\left|\lambda_{2}\right| \geq \cdots$, with $\left|\lambda_{k}\right|-\left|\lambda_{k+1}\right| \geq 2 \delta$. Let $B$ be a symmetric matrix such that $\|B\|<\delta$. Let $A_{k}$ and $(A+B)_{k}$ be the spaces spanned by the top $k$ eigenvectors of the respective matrices. Then

$$
\left\|I_{k}-A_{k}^{\top}(A+B)_{k}\right\| \leq \frac{\|B\|}{\delta} .
$$

Proof. See [13]. 
Now we are ready for the proof. Recall that $Y=X+Z$, we can write

$$
\begin{aligned}
Y Y^{\top} & =X X^{\top}+X Z^{\top}+X^{\top}+Z Z^{\top} \\
& =X X^{\top}+X Z^{\top}+Z X^{\top}+\left(Z Z^{\top}-\mathbb{E} Z Z^{\top}\right)+\mathbb{E} Z Z^{\top} .
\end{aligned}
$$

Since $\mathbb{E} Z Z^{\top}=\left(\sum_{j=1}^{p} \sigma_{j}^{2}\right) I$, the leading eigenvector of $Y^{\top} Y$ (i.e., $\hat{v}$ ) is the same as that of

$$
X X^{\top}+X Z^{\top}+Z X^{\top}+\left(Z Z^{\top}-\mathbb{E} Z Z^{\top}\right) .
$$

Since $\frac{1}{\sqrt{n}} l$ is the leading eigenvector of $X^{\top} X$, it follows that

$$
\begin{aligned}
& \mathbb{E} \mathcal{M}(l, \hat{l}) \stackrel{\text { Lemma }}{\leq}{ }^{5.6} \mathbb{E} \min _{ \pm}\left\|\frac{1}{\sqrt{n}} l \pm \hat{l}\right\|_{2}^{2} \leq \frac{\mathbb{E}\left\|X Z^{\top}+Z X^{\top}+Z Z^{\top}-\mathbb{E} Z Z^{\top}\right\|}{n\|\mu\|_{2}^{2}} \\
& \leq \frac{2 \mathbb{E}\left\|Z X^{\top}\right\|+\mathbb{E}\left\|Z^{\top} Z-\mathbb{E} Z^{\top} Z\right\|}{n\|\mu\|_{2}^{2}} \\
& \stackrel{\text { Lemma }}{\lesssim} .7 \frac{n\|\mu\|_{2} \sigma_{*}+\mathbb{E}\left\|Z^{\top} Z-\mathbb{E} Z^{\top} Z\right\|}{n\|\mu\|_{2}^{2}} \\
& \stackrel{\text { Theorem } 3.13}{\lesssim} \frac{n\|\mu\| \sigma_{*}+\sqrt{n \sum_{i=1}^{p} \sigma_{i}^{4}}+n \sigma_{*}^{2}}{n\|\mu\|_{2}^{2}} .
\end{aligned}
$$

Proof of Theorem 4.2. We only need to prove the lower bound under the following two situations:

- when $\lambda \leq c_{1} \sigma_{*}$, there exists $\left\{\sigma_{i}\right\}_{i=1}^{p}$ such that $\max _{i} \sigma_{i} \leq \sigma_{*}, \sum_{i} \sigma_{i}^{4} \leq \tilde{\sigma}^{4}$ and the lower bound holds;

- when $\lambda \leq c_{2} \tilde{\sigma} / n^{1 / 4}$, there exists $\left\{\sigma_{i}\right\}_{i=1}^{p}$ such that $\max _{i} \sigma_{i} \leq \sigma_{*}, \sum_{i} \sigma_{i}^{4} \leq \tilde{\sigma}^{4}$ and the lower bound holds.

We start with the first case. We specify $\sigma_{1}=\sigma_{*}$ and take $\sigma_{2}, \ldots, \sigma_{p}$ to be arbitrary values that satisfy the constraint of $\mathcal{P}_{\lambda, l}\left(\sigma_{*}, \tilde{\sigma}\right)$. Consider the metric space $\{-1,1\}^{n}$ with the metric

$$
\mathcal{M}\left(l^{(1)}, l^{(2)}\right)=\frac{1}{n} \min \left\{\left|i: l_{i}^{(1)} \neq l_{i}^{(2)}\right|,\left|i: l_{i}^{(i)} \neq-l_{i}^{(2)}\right|\right\}
$$

By [35, Lemma 4], when $n \geq 6$, we can find some constant $c_{0}$, such that there exists a subset $\left\{l^{(1)}, \ldots, l^{(N)}\right\} \subset\{-1,1\}^{n}$ satisfying

$$
\mathcal{M}\left(l^{\left(i_{1}\right)}, l^{\left(i_{2}\right)}\right) \geq 1 / 3, \quad \forall 1 \leq i_{1}<i_{2} \leq N
$$

and $N \geq \exp \left(c_{0} n\right)$. Let $Y^{(i)}=\mu\left(l^{(i)}\right)^{\top}+Z \in \mathbb{R}^{p \times n}$, where $Z_{i j} \stackrel{i n d}{\sim} N\left(0, \sigma_{i}^{2}\right)$. Let $\mu=$ $[\lambda, 0, \cdots, 0]^{\top}$, then the KL-divergence between $Y^{\left(i_{i}\right)}$ and $Y^{\left(i_{2}\right)}$ for $i_{1} \neq i_{2}$ is

$$
D_{K L}\left(Y^{\left(i_{1}\right)} \mid Y^{\left(i_{2}\right)}\right)=\frac{1}{2} \sum_{j=1}^{p} \sigma_{j}^{-2} \mu_{j}^{2}\left\|l^{\left(i_{1}\right)}-l^{\left(i_{2}\right)}\right\|_{2}^{2} \leq 4 n \Sigma_{j=1}^{p} \sigma_{j}^{-2} \mu_{j}^{2}=4 n \sigma_{1}^{-2} \lambda^{2}=4 n \lambda^{2} / \sigma_{*}^{2} .
$$

By the generalized Fano's lemma, we have

$$
\inf _{\hat{l}} \sup _{\mathcal{P}_{l, \lambda}\left(\sigma_{*}, \tilde{\sigma}\right)} \mathbb{E} \mathcal{M}(l, \hat{l}) \geq \frac{1}{3}\left(1-\frac{4 n \lambda^{2} / \sigma_{*}^{2}+\log 2}{c_{0} n}\right) \geq \frac{1}{4}
$$

In the last inequality we use the assumption that $\lambda \leq c_{1} \sigma_{*}^{2}$ for some sufficiently small constant $c_{1}$.

Now we consider the second situation. We specify $\sigma_{1}^{4}=\sigma_{2}^{4}=\ldots=\sigma_{p}^{4}=\frac{\tilde{\sigma}^{4}}{p}$. When the variance structure reduces to a homoskedastic structure, we have the following lower bound result which is already established. 
Lemma 5.9. Suppose $\sigma_{1}^{2}=\cdots=\sigma_{p}^{2}=1$, there exists $c_{2}, C$ such that if $n \geq C$,

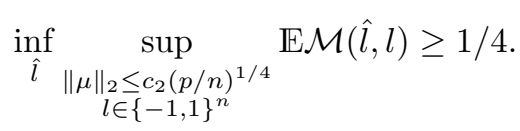

Proof. See [11, Theorem 6].

Based on Lemma 5.9 and homoskedasticity of $\mu$ and $\sigma$, if we set $\lambda<\frac{c_{2} \tilde{\sigma}}{p^{1 / 4}} \cdot\left(\frac{p}{n}\right)^{1 / 4}=$ $c_{2} \tilde{\sigma} / n^{1 / 4}$ in our setting, we obtain

$$
\inf _{\hat{l}} \sup _{\mathcal{P}_{l, \lambda}\left(\sigma_{*}, \tilde{\sigma}\right)} \mathbb{E} \mathcal{M}(l, \hat{l}) \geq 1 / 4
$$

This finishes the proof.

\section{References}

[1] Oskari H Ajanki, László Erdős, and Torben Krüger, Universality for general wigner-type matrices, Probability Theory and Related Fields 169 (2017), no. 3-4, 667-727. MR3719056

[2] Greg W Anderson, Alice Guionnet, and Ofer Zeitouni, An introduction to random matrices, vol. 118, Cambridge university press, 2010. MR2760897

[3] Zhidong D Bai, Convergence rate of expected spectral distributions of large random matrices. part ii. sample covariance matrices, The Annals of Probability 21 (1993), no. 2, 649-672. MR1217560

[4] Afonso S Bandeira and Ramon van Handel, Sharp nonasymptotic bounds on the norm of random matrices with independent entries, The Annals of Probability 44 (2016), no. 4, 2479-2506. MR3531673

[5] Zhigang Bao, Xiucai Ding, and Ke Wang, Singular vector and singular subspace distribution for the matrix denoising model, The Annals of Statistics to appear (2020). MR4206682

[6] Necdet Batır, Bounds for the gamma function, Results in Mathematics 72 (2017), no. 1-2, 865-874. MR3684463

[7] Florent Benaych-Georges and Raj Rao Nadakuditi, The singular values and vectors of low rank perturbations of large rectangular random matrices, Journal of Multivariate Analysis 111 (2012), 120-135. MR2944410

[8] Adrian N Bishop, Pierre Del Moral, and Angèle Niclas, An introduction to wishart matrix moments, Foundations and Trends in Machine Learning 11 (2018), no. 2.

[9] Stéphane Boucheron, Olivier Bousquet, Gábor Lugosi, Pascal Massart, et al., Moment inequalities for functions of independent random variables, The Annals of Probability 33 (2005), no. 2, 514-560. MR2123200

[10] Stéphane Boucheron, Gábor Lugosi, and Pascal Massart, Concentration inequalities: A nonasymptotic theory of independence, Oxford university press, 2013. MR3185193

[11] T Tony Cai and Anru Zhang, Rate-optimal perturbation bounds for singular subspaces with applications to high-dimensional statistics, The Annals of Statistics 46 (2018), no. 1, 60-89. MR3766946

[12] T Tony Cai, Cun-Hui Zhang, and Harrison H Zhou, Optimal rates of convergence for covariance matrix estimation, The Annals of Statistics 38 (2010), no. 4, 2118-2144. MR2676885

[13] Chandler Davis and William Morton Kahan, The rotation of eigenvectors by a perturbation. iii, SIAM Journal on Numerical Analysis 7 (1970), no. 1, 1-46. MR0264450

[14] David Donoho and Matan Gavish, Minimax risk of matrix denoising by singular value thresholding, The Annals of Statistics 42 (2014), no. 6, 2413-2440. MR3269984

[15] Laura Florescu and Will Perkins, Spectral thresholds in the bipartite stochastic block model, Conference on Learning Theory, 2016, pp. 943-959. 
[16] Trevor Hastie, Robert Tibshirani, and Jerome Friedman, The elements of statistical learning: data mining, inference, and prediction, Springer Science \& Business Media, 2009. MR2722294

[17] David Hong, Laura Balzano, and Jeffrey A Fessler, Asymptotic performance of pca for highdimensional heteroscedastic data, Journal of multivariate analysis 167 (2018), 435-452. MR3830656

[18] Vladimir Koltchinskii and Karim Lounici, Concentration inequalities and moment bounds for sample covariance operators, Bernoulli 23 (2017), no. 1, 110-133. MR3556768

[19] Arun Kumar Kuchibhotla and Abhishek Chakrabortty, Moving beyond sub-gaussianity in high-dimensional statistics: Applications in covariance estimation and linear regression, arXiv preprint arXiv:1804.02605 (2018).

[20] Rafał Latała, Ramon van Handel, and Pierre Youssef, The dimension-free structure of nonhomogeneous random matrices, arXiv preprint arXiv:1711.00807 (2017). MR3878726

[21] Marc Lelarge, Laurent Massoulié, and Jiaming Xu, Reconstruction in the labelled stochastic block model, IEEE Transactions on Network Science and Engineering 2 (2015), no. 4, 152163. MR3453283

[22] Lydia T Liu, Edgar Dobriban, and Amit Singer, e PCA: High dimensional exponential family PCA, arXiv preprint arXiv:1611.05550 (2016). MR3875695

[23] Sahand Negahban, Sewoong Oh, and Devavrat Shah, Rank centrality: Ranking from pairwise comparisons, Operations Research 65 (2017), no. 1, 266-287. MR3613103

[24] Mark EJ Newman, Spectral methods for community detection and graph partitioning, Physical Review E 88 (2013), no. 4, 042822.

[25] Joseph Salmon, Zachary Harmany, Charles-Alban Deledalle, and Rebecca Willett, Poisson noise reduction with non-local PCA, Journal of mathematical imaging and vision 48 (2014), no. 2, 279-294. MR3152105

[26] Andrey A Shabalin and Andrew B Nobel, Reconstruction of a low-rank matrix in the presence of gaussian noise, Journal of Multivariate Analysis 118 (2013), 67-76. MR3054091

[27] Ann-Christine Syvänen, Accessing genetic variation: genotyping single nucleotide polymorphisms, Nature Reviews Genetics 2 (2001), no. 12, 930-942.

[28] Terence Tao, Topics in random matrix theory, vol. 132, American Mathematical Soc., 2012. MR2906465

[29] Joel A Tropp, The expected norm of a sum of independent random matrices: An elementary approach, High Dimensional Probability VII, Springer, 2016, pp. 173-202. MR3565264

[30] Ramon van Handel, On the spectral norm of gaussian random matrices, Transactions of the American Mathematical Society 369 (2017), no. 11, 8161-8178. MR3695857

[31] Roman Vershynin, Introduction to the non-asymptotic analysis of random matrices, Compressed Sensing: Theory and Applications (2009), 210-268. MR2963170

[32] Roman Vershynin, Spectral norm of products of random and deterministic matrices, Probability theory and related fields 150 (2011), no. 3-4, 471-509. MR2824864

[33] Mariia Vladimirova, Stéphane Girard, Hien Nguyen, and Julyan Arbel, Sub-weibull distributions: generalizing sub-gaussian and sub-exponential properties to heavier-tailed distributions, arXiv preprint arXiv:1905.04955 (2019). MR4193421

[34] Hermann Weyl, Das asymptotische verteilungsgesetz der eigenwerte linearer partieller differentialgleichungen (mit einer anwendung auf die theorie der hohlraumstrahlung), Mathematische Annalen 71 (1912), no. 4, 441-479. MR1511670

[35] Bin Yu, Assouad, Fano, and Le Cam, Festschrift for Lucien Le Cam, Springer, 1997, pp. 423435. MR1462963

[36] Anru Zhang, T Tony Cai, and Yihong Wu, Heteroskedastic PCA: Algorithm, optimality, and applications, arXiv preprint arXiv:1810.08316 (2018). MR4382008

[37] Anru Zhang and Yuchen Zhou, On the non-asymptotic and sharp lower tail bounds of random variables, arXiv preprint arXiv:1810.09006 (2018). MR4193419 


\section{A Proofs of technical lemmas}

We collect the proofs of Lemma 5.2, 5.3, and 5.5 in this section.

Proof of Lemma 5.2. We first consider the proof of (5.17). Note that if $G \sim N(0,1)$,

$$
\mathbb{E} G^{d}= \begin{cases}(d-1) ! !, & d \geq 0, \text { and } d \text { is even; } \\ 0, & d \geq 0, \text { and } d \text { is odd. }\end{cases}
$$

In addition, $(-1) ! !=1,(-3) ! !=-1$. When $\alpha$ is odd, only odd moments of $G$ appear in the expansion of $G^{\alpha}\left(G^{2}-1\right)^{\beta}$, then clearly $\mathbb{E} G^{\alpha}\left(G^{2}-1\right)^{\beta}=0$. When $\alpha$ is even and $\alpha+2 \beta \geq 4$,

$$
\begin{aligned}
\mathbb{E} G^{\alpha}\left(G^{2}-1\right)^{\beta} & =\sum_{j=0}^{\beta} \mathbb{E} G^{\alpha+2 \beta-2 j}(-1)^{j}\left(\begin{array}{l}
\beta \\
j
\end{array}\right)=\sum_{j=0}^{\beta}(-1)^{j}(\alpha+2 \beta-2 j-1) ! ! \cdot \frac{\beta !}{(\beta-j) ! j !} \\
& \geq \sum_{\substack{0 \leq j \leq \beta \\
j \text { is even }}}\left\{\frac{(\alpha+2 \beta-2 j-1) ! ! \beta !}{(\beta-j) ! j !}-\frac{(\alpha+2 \beta-2(j+1)-1) ! ! \beta !}{(\beta-j-1) !(j+1) !}\right\} \\
& =\sum_{\substack{0 \leq j \leq \beta \\
j \text { is even }}} \frac{(\alpha+2 \beta-2 j-3) ! ! \beta !}{(\beta-j) !(j+1) !} \cdot\{(\alpha+2 \beta-2 j-1)(j+1)-(\beta-j)\}
\end{aligned}
$$

- If $j=\beta$,

$$
\begin{aligned}
& \frac{(\alpha+2 \beta-2 j-3) ! ! \beta !}{(\beta-j) !(j+1) !} \cdot\{(\alpha+2 \beta-2 j-1)(j+1)-(\beta-j)\} \\
= & \frac{(\alpha+2 \beta-2 j-3) ! ! \beta !}{(\beta-j) !(j+1) !} \cdot(\alpha+2 \beta-2 j-1)(j+1) \geq 0
\end{aligned}
$$

- If $\beta-1 \geq j \geq \frac{\beta-1}{2}$,

$$
(\alpha+2 \beta-2 j-1)(j+1) \geq(\alpha+2 \beta-2(\beta-1)-1)\left(\frac{\beta-1}{2}+1\right) \geq \frac{\beta+1}{2} \geq \beta-j
$$

- if $0 \leq j<\frac{\beta-1}{2}$,

$$
(\alpha+2 \beta-2 j-1)(j+1) \geq \alpha+2 \beta-(\beta-1)-1 \geq \beta-j .
$$

Thus, we always have

$$
\frac{(\alpha+2 \beta-2 j-3) ! ! \beta !}{(\beta-j) !(j+1) !} \cdot\{(\alpha+2 \beta-2 j-1)(j+1)-(\beta-j)\} \geq 0, \quad \forall 0 \leq j \leq \beta, j \text { is even, }
$$

and

$$
\begin{aligned}
\mathbb{E} G^{\alpha}\left(G^{2}-1\right)^{\beta} & \geq \sum_{j=0}^{0} \frac{(\alpha+2 \beta-2 j-3) ! ! \beta !}{(\beta-j) !(j+1) !} \cdot\{(\alpha+2 \beta-2 j-1)(j+1)-(\beta-j)\} \\
& =(\alpha+2 \beta-3) ! ! \cdot(\alpha+\beta-1),
\end{aligned}
$$

which has finished the proof of (5.17).

Next we consider the upper bound of $\mathbb{E} G^{\alpha}\left(G^{2}-1\right)^{\beta}$.

$$
\begin{aligned}
& \mathbb{E} G^{\alpha}\left(G^{2}-1\right)^{\beta}=\sum_{j=0}^{\beta} \mathbb{E} G^{\alpha+2 \beta-2 j}(-1)^{j}\left(\begin{array}{c}
\beta \\
j
\end{array}\right)=\sum_{j=0}^{\beta}(-1)^{j}(\alpha+2 \beta-2 j-1) ! ! \cdot \frac{\beta !}{(\beta-j) ! j !} \\
\leq & (\alpha+2 \beta-1) ! !-\sum_{\substack{0 \leq j \leq \beta \\
j \text { is odd }}}\left\{\frac{(\alpha+2 \beta-2 j-1) ! ! \beta !}{(\beta-j) ! j !}-\frac{(\alpha+2 \beta-2(j+1)-1) ! ! \beta !}{(\beta-j-1) !(j+1) !}\right\} \\
= & (\alpha+2 \beta-1) ! !-\sum_{\substack{0 \leq j \leq \beta \\
j \text { is odd }}} \frac{(\alpha+2 \beta-2 j-3) ! ! \beta !}{(\beta-j) !(j+1) !} \cdot\{(\alpha+2 \beta-2 j-1)(j+1)-(\beta-j)\}
\end{aligned}
$$




\section{Heteroskedastic Wishart-type concentration}

Similarly as the previous argument, we can show for any odd $1 \leq j \leq \beta$,

$$
\frac{(\alpha+2 \beta-2 j-3) ! ! \beta !}{(\beta-j) !(j+1) !} \cdot\{(\alpha+2 \beta-2 j-1)(j+1)-(\beta-j)\} \geq 0,
$$

thus,

$$
\mathbb{E} G^{\alpha}\left(G^{2}-1\right)^{\beta} \leq(\alpha+2 \beta-1) ! !
$$

Then we consider the proof of sub-Gaussian case (5.19). When $\alpha$ is odd, the statement clearly holds as $Z^{\alpha}\left(Z^{2}-1\right)^{\beta}$ has symmetric distribution then $\mathbb{E} Z^{\alpha}\left(Z^{2}-1\right)^{\beta}=0$. When $\alpha$ is even, since $Z^{2} \geq 0$, we must have $\left|Z^{2}-1\right|=\max \left\{Z^{2}-1,1-Z^{2}\right\} \leq Z^{2} \vee 1$, thus

$$
\begin{aligned}
\left|\mathbb{E} Z^{\alpha}\left(Z^{2}-1\right)^{\beta}\right| & \leq\left|\mathbb{E} Z^{\alpha}\left(Z^{2}-1\right)^{\beta} 1_{\{|Z| \leq 1\}}+\mathbb{E} Z^{\alpha}\left(Z^{2}-1\right)^{\beta} 1_{\{|Z|>1\}}\right| \\
& \leq 1+\mathbb{E} Z^{\alpha}\left|Z^{2}\right|^{\beta}=\mathbb{E}|Z|^{\alpha+2 \beta}+1 .
\end{aligned}
$$

Since $\mathbb{E} Z^{2}=1$, we have $\kappa \geq 1 / \sqrt{2}$. Thus,

$$
\mathbb{E}|Z|^{\alpha+2 \beta}+1 \leq(\kappa+1)^{\alpha+2 \beta}(\alpha+2 \beta)^{(\alpha+2 \beta) / 2} \leq(3 \kappa)^{\alpha+2 \beta}(\alpha+2 \beta)^{(\alpha+2 \beta) / 2} .
$$

It is easy to see (5.19) holds when $\alpha+2 \beta \leq 2$.

When $\alpha+2 \beta \geq 4$, by the relationship between double factorial and Gamma function ${ }^{1}$ and the lower bound of Gamma function [6], we have

$$
\begin{aligned}
& (\alpha+2 \beta-3) ! !(\alpha+\beta-1)=\frac{2^{\alpha / 2+\beta-1}}{\sqrt{\pi}} \Gamma\left(\frac{\alpha}{2}+\beta-1+\frac{1}{2}\right)(\alpha+\beta-1) \\
\geq & \frac{2^{\alpha / 2+\beta-1}(\alpha+\beta-1)}{\sqrt{\pi}} \cdot \sqrt{2 \pi} x^{x} e^{-x}\left(x^{2}+x / 3+0.04\right)^{1 / 4} \\
\geq & C^{-(\alpha+2 \beta)} \cdot(\alpha+2 \beta)^{(\alpha+2 \beta) / 2}=(C \kappa)^{\alpha+2 \beta}(\alpha+2 \beta)^{(\alpha+2 \beta) / 2} \geq \mathbb{E} Z^{\alpha}\left(Z^{2}-1\right)^{\beta} .
\end{aligned}
$$

Here, $x=\frac{\alpha+2 \beta-3}{2}$.

Proof of Lemma 5.3. Firstly we have

$$
\begin{aligned}
& \mathbb{E} F_{i j}^{\alpha}\left(F_{i j}^{2}-1\right)^{\beta}=\sum_{j=0}^{\beta} \mathbb{E} F_{i j}^{\alpha+2 \beta-2 j}(-1)^{j}\left(\begin{array}{c}
\beta \\
j
\end{array}\right) \\
& \quad=\frac{1}{\sqrt{\pi}} \sum_{j=0}^{\beta}(-1)^{j} \frac{\beta !}{(\beta-j) ! j !}\left(x_{j}-1\right) ! ! \cdot 2^{\frac{(b-1) x_{j}}{2}} \Gamma\left(\frac{(b-1) x_{j}+1}{2}\right) \\
& \geq \frac{1}{\sqrt{\pi}} \sum_{j \leq \beta}\left\{\frac{\beta !}{(\beta-j) ! j !}\left(x_{j}-1\right) ! ! \cdot 2^{\frac{(b-1) x_{j}}{2}} \Gamma\left(\frac{(b-1) x_{j}+1}{2}\right)\right. \\
& \geq \frac{1}{\sqrt{\pi}} \sum_{\substack{j \leq \beta \\
\text { is even }}} \frac{-\beta !}{(\beta-j) !(j+1) !}\left(x_{j}-3\right) ! ! 2^{\frac{(b-1)\left(x_{j}-2\right)}{2}} \Gamma\left(\frac{(b-1)\left(x_{j}-2\right)+1}{2}\right) \\
& \left.\cdot(j-1)\left(x_{j}-1\right)-(\beta-j)\right],
\end{aligned}
$$

where $x_{j}:=\alpha+2 \beta-2 j$ and the last inequality comes from the strictly increasing property of Gamma function. By the proof of Lemma 5.2, we know

$$
\frac{\beta !}{(\beta-j) !(j+1) !}\left(x_{j}-3\right) ! ! \cdot\left((j+1)\left(x_{j}-1\right)-(\beta-j)\right) \geq 0 .
$$

\footnotetext{
${ }^{1}$ See https://en.wikipedia.org/wiki/Double_factorial
} 
Thus,

$$
\begin{aligned}
\mathbb{E} F_{i j}^{\alpha}\left(F_{i j}^{2}-1\right)^{\beta} & \geq \frac{\alpha+\beta-1}{\sqrt{\pi}}(\alpha+2 \beta-3) ! ! \cdot 2^{\frac{(b-1)(\alpha+2 \beta-2)}{2}} \Gamma\left(\frac{(b-1)(\alpha+2 \beta-2)+1}{2}\right) \\
& =\frac{1}{\pi} 2^{\frac{b(\alpha+2 \beta-2)}{2}} \Gamma\left(\frac{\alpha+2 \beta-1}{2}\right) \Gamma\left(\frac{(b-1)(\alpha+2 \beta-2)+1}{2}\right) .
\end{aligned}
$$

When $\alpha+2 \beta \geq\left(2+\frac{3}{b-1}\right) \vee 5$, by the lower bound of Gamma function [6], we further have

$$
\begin{aligned}
\mathbb{E} F_{i j}^{\alpha}\left(F_{i j}^{2}-1\right)^{\beta} & \geq 2 \cdot 2^{\frac{b(\alpha+2 \beta-2)}{2}} x^{x} e^{-x}\left(x^{2}+\frac{x}{3}+0.04\right)^{1 / 4} y^{y} e^{-y}\left(y^{2}+\frac{y}{3}+0.04\right)^{1 / 4} \\
& \geq\left(c_{b}\right)^{\alpha+2 \beta} \cdot(\alpha+2 \beta)^{(\alpha+2 \beta) / 2} \cdot((b-1)(\alpha+2 \beta))^{(b-1)(\alpha+2 \beta) / 2} \\
& \geq\left(c_{b}^{\prime}\right)^{\alpha+2 \beta} \cdot(\alpha+2 \beta)^{b(\alpha+2 \beta) / 2}
\end{aligned}
$$

where $x=\frac{\alpha+2 \beta-3}{2}, y=\frac{(b-1)(\alpha+2 \beta-2)-1}{2}$ and $c_{b}>0$ is some constant that only depends on $b$.

When $2 \leq \alpha+2 \beta<\left(2+\frac{3}{b-1}\right) \vee 5$, we can find another universal constant $c_{b}^{\prime \prime}$ such that

$$
\frac{1}{\pi} 2^{\frac{b(\alpha+2 \beta-2)}{2}} \Gamma\left(\frac{\alpha+2 \beta-1}{2}\right) \Gamma\left(\frac{(b-1)(\alpha+2 \beta-2)+1}{2}\right) \geq\left(c_{b}^{\prime \prime}\right)^{\alpha+2 \beta} \cdot(\alpha+2 \beta)^{b(\alpha+2 \beta) / 2} .
$$

In conclusion, we proved that

$$
\mathbb{E} F_{i j}^{\alpha}\left(F_{i j}^{2}-1\right)^{\beta} \geq\left(C_{b} \kappa\right)^{\alpha+2 \beta} \cdot \mathbb{E} E_{i j}^{\alpha}\left(E_{i j}^{2}-1\right)^{\beta}
$$

for any $\alpha, \beta \geq 0$. Thus (5.21) is proved.

Proof of Lemma 5.5. If either $\left(x_{1}, x_{3}, x_{5}\right)=(0,0,0)$ or $\left(x_{2}, x_{4}, x_{5}\right)=(0,0,0)$, the statement (5.33) immediately follows from the proof of Lemma 5.2 and the statement of (5.34) becomes identity; if either $x_{1}+x_{5}$ or $x_{2}+x_{5}$ is odd, the left hand side of (5.33) (5.34) are zero since $Z_{1}$ and $Z_{2}$ are symmetric distributed and independent. Meanwhile, the right hand side of (5.33) is non-negative (Lemma 5.2), thus (5.33) holds if either $x_{1}+x_{5}$ or $x_{2}+x_{5}$ is odd. When $\left(x_{1}, x_{3}, x_{5}\right)=(0,1,0)$ (or $\left.\left(x_{2}, x_{4}, x_{5}\right)=(0,1,0)\right)$, by similar arguments one can show (5.33) holds.

Thus we only need to prove the inequality when both $x_{1}+x_{5}$ and $x_{2}+x_{5}$ are even, and

$$
x_{1}+x_{5}+x_{3} \geq 2, \text { and } x_{2}+x_{5}+x_{4} \geq 2 .
$$

By Lemma 5.2, we have

$$
\begin{aligned}
& \left|\mathbb{E} Z_{1}^{x_{1}+x_{5}} Z_{2}^{x_{2}+x_{5}}\left(Z_{1}^{2}-1\right)^{x_{3}}\left(Z_{2}^{2}-1\right)^{x_{4}}\right| \\
= & \left|\left(\mathbb{E} Z_{1}^{x_{1}+x_{5}}\left(Z_{1}^{2}-1\right)^{x_{3}}\right) \cdot\left(\mathbb{E} Z_{2}^{x_{2}+x_{5}}\left(Z_{2}^{2}-1\right)^{x_{4}}\right)\right| \\
\leq & (C \kappa)^{x_{1}+x_{2}+2\left(x_{3}+x_{4}+x_{5}\right)} \cdot\left|\left(\mathbb{E} G^{x_{1}+x_{5}}\left(G^{2}-1\right)^{x_{3}}\right) \cdot\left(\mathbb{E} G^{x_{2}+x_{5}}\left(G^{2}-1\right)^{x_{4}}\right)\right| \\
\leq & (C \kappa)^{x_{1}+x_{2}+2\left(x_{3}+x_{4}+x_{5}\right)} \cdot\left(x_{1}+x_{5}+2 x_{3}-1\right) ! ! \cdot\left(x_{2}+x_{5}+2 x_{4}-1\right) ! ! .
\end{aligned}
$$

Since for any odd positive integers $x, y, x ! ! \cdot y ! ! \leq(x+y-1) ! !$, we have

$$
\left(x_{1}+x_{5}+2 x_{3}-1\right) ! ! \cdot\left(x_{2}+x_{5}+2 x_{4}-1\right) ! ! \leq\left(x_{1}+x_{2}+2\left(x_{3}+x_{4}+x_{5}\right)-3\right) ! !
$$

Therefore,

$$
\begin{aligned}
&\left|\mathbb{E} Z_{1}^{x_{1}+x_{5}} Z_{2}^{x_{2}+x_{5}}\left(Z_{1}^{2}-1\right)^{x_{3}}\left(Z_{2}^{2}-1\right)^{x_{4}}\right| \\
& \leq(C \kappa)^{x_{1}+x_{2}+2\left(x_{3}+x_{4}+x_{5}\right)} \cdot\left(x_{1}+x_{2}+2\left(x_{3}+x_{4}+x_{5}\right)-3\right) ! ! \cdot\left(x_{1}+x_{2}+x_{3}+x_{4}+x_{5}\right) \\
& \stackrel{\text { Lemma } 5.2}{\leq}(C \kappa)^{x_{1}+x_{2}+2\left(x_{3}+x_{4}+x_{5}\right)} \cdot \mathbb{E}\left(G^{2}-1\right)^{x_{3}+x_{4}+x_{5}} G^{x_{1}+x_{2}},
\end{aligned}
$$


Heteroskedastic Wishart-type concentration

which has finished the proof of (5.33).

If $Z_{1}, Z_{2}, G$ are standard Gaussian, by Lemma 5.2,

$$
\begin{array}{rl} 
& \left|\mathbb{E} Z_{1}^{x_{1}} Z_{2}^{x_{2}}\left(Z_{1}^{2}-1\right)^{x_{3}}\left(Z_{2}^{2}-1\right)^{x_{4}}\left(Z_{1} Z_{2}\right)^{x_{5}}\right| \\
= & \left|\left(\mathbb{E} Z_{1}^{x_{1}+x_{5}}\left(Z_{1}^{2}-1\right)^{x_{3}}\right)\right| \cdot\left|\left(\mathbb{E} Z_{2}^{x_{2}+x_{5}}\left(Z_{2}^{2}-1\right)^{x_{4}}\right)\right| \\
\leq & \left(x_{1}+x_{5}+2 x_{3}-1\right) ! ! \cdot\left(x_{2}+x_{5}+2 x_{4}-1\right) ! ! \\
\leq & \left(x_{1}+x_{2}+2\left(x_{3}+x_{4}+x_{5}\right)-3\right) ! ! \cdot\left(x_{1}+x_{2}+x_{3}+x_{4}+x_{5}\right) \\
\stackrel{\text { Lemma }}{5.2} & \mathbb{E} G^{x_{1}+x_{2}}\left(G^{2}-1\right)^{x_{3}+x_{4}+x_{5}},
\end{array}
$$

which has finished the proof of (5.34). 